\title{
On the continuity of solutions to doubly singular parabolic equations
}

\section{Qifan $\mathrm{Li}^{1}$}

Received: 13 December 2018 / Accepted: 11 November 2019 / Published online: 23 November 2019 (c) Fondazione Annali di Matematica Pura ed Applicata and Springer-Verlag GmbH Germany, part of Springer Nature 2019

\section{Abstract}

This paper considers certain quasilinear parabolic equations with one singularity which occurs in the time derivative. A model equation is

$$
\partial_{t} \beta(u)-\operatorname{div}|D u|^{p-2} D u \ni 0,
$$

where $\beta: \mathbb{R} \rightarrow \mathbb{R}$ is monotonically increasing and discontinuous at the origin. We show that bounded weak solutions are locally continuous in the range

$$
2-\varepsilon_{0} \leq p<2
$$

provided that $\varepsilon_{0}>0$ is sufficiently small, and the continuity is stable as $p \rightarrow 2$.

Keywords Two-phase Stefan problem · Singular parabolic equations $\cdot$ Phase transition

Mathematics Subject Classification Primary 35R05 - 35R35 - 35D30; Secondary 35K59 . $35 \mathrm{~K} 92$

\section{Introduction}

The aim of this paper is to establish a continuity result for solutions to singular parabolic equations related to two-phase Stefan problems. More precisely, we consider quasilinear parabolic equations of the form

$$
\partial_{t} \beta(u)-\operatorname{div} A(x, t, u, D u) \ni 0,
$$

with $u: \Omega_{T} \rightarrow \mathbb{R}$, in a bounded domain $\Omega_{T}=\Omega \times(0, T)$, where $\Omega \subset \mathbb{R}^{N}$. Throughout the paper, we keep $N \geq 3$. The function $\beta(\cdot)$ is defined by

Qifan Li

qifan_li@yahoo.com

1 Department of Mathematics, School of Sciences, Wuhan University of Technology, 122 Luoshi Road, Wuhan 430070, Hubei, People's Republic of China 


$$
\beta(r)= \begin{cases}r, & r>0, \\ {[-\bar{v}, 0],} & r=0, \\ r-\bar{v}, & r<0\end{cases}
$$

where $\bar{v}>0$ is a given constant. The vector field $A(x, t, u, \zeta)$ is measurable in $\Omega_{T} \times \mathbb{R} \times \mathbb{R}^{N}$ and satisfies the structure conditions:

$$
\left\{\begin{array}{l}
A(x, t, u, \zeta) \cdot \zeta \geq C_{0}|\zeta|^{p}, \\
|A(x, t, u, \zeta)| \leq C_{1}|\zeta|^{p-1},
\end{array}\right.
$$

where $C_{0}$ and $C_{1}$ are given positive constants. No attempt is made here to discuss equations with lower-order terms. This study is motivated by phase transitions for material obeying a Fourier's law (see [7-9]). The continuity of weak solutions for the case $A(x, t, u, D u)=D u$ was first proved by Caffarelli and Evans [2]. DiBenedetto [4] then extended this result to quasilinear equations with a general structure. The proof is based on De Giorgi's technique and a construction of logarithmic functions to prove the expansion of positivity in the time direction. Furthermore, Urbano [10] established the continuity result for the degenerate case $p \geq 2$ using De Giorgi's technique combined with the intrinsic scaling method. This idea dates back at least as far as [4].

The treatment of the singular case $p<2$ is considerably more difficult. Henriques and Urbano [6] studied this problem and introduced intrinsic parabolic cylinders of the form

$$
\left[(\bar{x}, \bar{t})+Q\left(d R^{p}, d_{*} R\right)\right], \quad d=\left(\frac{\omega}{2}\right)^{(1-p)(2-p)} \quad \text { and } \quad d_{*}=\left(\frac{\omega}{2^{n_{*}+1}}\right)^{p-2}
$$

and used this kind of cylinder to address alternative arguments. However, we note that there is a mistake in the proof of Lemma 1 ([6, page 930, line 8]). The integral involving the term $\left|\nabla\left(\bar{\theta}-\bar{k}_{n}\right)-\bar{\xi}_{n}\right|^{p}$ must be multiplied by the factor $c_{0}^{-p}$ due to the chain rule. If we multiply this factor, the constant $\nu_{0}$ in [6, page 927] will depend upon $2^{-n_{*}}$ which is so small that we cannot determine the number $n_{*}$ in terms of $v_{0}$ (see [6, Lemma 4 , page 938 , line-1]). Of course, this is not meant to diminish the importance of Henriques and Urbano's pioneering work but rather to highlight that it does not resolve the problem and that a correct proof is needed.

In this work, we attempt to find a solution to the difficulty mentioned above. We use the following intrinsic parabolic cylinders

$$
\begin{gathered}
{\left[(\bar{x}, \bar{t})+Q\left(d R^{p}, d_{*} R\right)\right], \quad d=\left(\frac{\omega}{2^{m_{1}}}\right)^{(1-p)(2-p)} \text { and }} \\
d_{*}=\left(\frac{\omega}{2^{m_{2}}}\right)^{p-2} \quad \text { where } m_{1}=\frac{p m_{2}}{p-1}
\end{gathered}
$$

for the alternative arguments. Since the length of the time interval is too large, we cannot simply use the idea from [6, Lemma 4] to determine the quantity $m_{2}$, and the analysis of the second alternative is considerably more delicate. To obtain the pointwise expansion of positivity in such a large time interval, we apply an iterative argument. However, our approach is effective only for the range $2-\varepsilon_{0} \leq p<2$, where $\varepsilon_{0}$ is a small positive constant depending only upon the data. We have to impose this artificial condition because the integrals involving the quantity $\left[\psi_{ \pm}^{\prime}\right]^{2-p}$, where $\psi_{ \pm}$is a logarithmic function, are too large in the singular range. Moreover, we can also show that the continuity is stable as $p \rightarrow 2$. We leave the proof for the full range $1<p<2$ to future work.

An outline of this paper is as follows. We provide some preliminary material and state the main result in Sect. 2, while Sect. 3 provides an exposition of Caccioppoli estimates. In 
Sect. 4, we introduce the intrinsic parabolic cylinders for the alternative arguments. Section 5 is devoted to the analysis of the first alternative. We obtain a decay estimate for the oscillation of the weak solution in a smaller cylinder. Subsequently, Sect. 6 is intended to prove a similar estimate in the second alternative. Finally, in Sect. 7, we complete the proof of the main result by a recursive argument.

\section{Preliminary material and main result}

We follow the notation of [5]. Throughout the paper, $\Omega$ denotes a bounded open set in $\mathbb{R}^{N}$ with $N \geq 3$ and $\Omega_{T}=\Omega \times(0, T)$ is the associated space-time cylinder. We let $D u=$ $\left(\partial_{x_{1}} u, \partial_{x_{2}} u, \ldots, \partial_{x_{N}} u\right)$ stand for differentiation with respect to the space variables and $\partial_{t} u$ denote the time derivative. The derivatives are taken in the weak sense. Points in $\mathbb{R}^{N+1}$ will be denoted by $(x, t)$, where $x \in \mathbb{R}^{N}$ and $t \in \mathbb{R}$. We use cubes of the form

$$
K_{r}=\left\{x=\left(x_{1}, x_{2}, \ldots, x_{N}\right) \in \mathbb{R}^{N}:\left|x_{i}\right| \leq r, i=1,2, \ldots, N\right\} .
$$

For the parabolic cylinders, we write $Q\left(r_{1}, r_{2}\right)=K_{r_{2}} \times\left(-r_{1}, 0\right)$. Let $\partial K_{r_{2}}$ be the boundary of $K_{r_{2}}$ and $\partial_{P} Q\left(r_{1}, r_{2}\right)=\left[\partial K_{r_{2}} \times\left(-r_{1}, 0\right)\right] \cup\left[K_{r_{2}} \times\{0\}\right]$ denote the parabolic boundary of $Q\left(r_{1}, r_{2}\right)$. Let $r^{\prime}>r>0$. We construct a piecewise smooth function, with $\zeta \equiv 1$ in $K_{r}$, $\operatorname{supp} \zeta \subset K_{r^{\prime}}$ and $|D \zeta| \leq\left(r^{\prime}-r\right)^{-1}$, by

$$
\zeta(x)=\prod_{i=1}^{N} \zeta_{i}\left(x_{i}\right)
$$

where $x=\left(x_{1}, x_{2}, \ldots, x_{N}\right)$ and

$$
\zeta_{i}\left(x_{i}\right)= \begin{cases}0, & \text { if }\left|x_{i}\right|>r^{\prime}, \\ \frac{x_{i}+r^{\prime}}{r^{\prime}-r}, & \text { if }-r^{\prime}<x_{i}<-r, \\ 1, & \text { if }\left|x_{i}\right| \leq r, \\ \frac{r^{\prime}-x_{i}}{r^{\prime}-r}, & \text { if } \quad r<x_{i}<r^{\prime} .\end{cases}
$$

We set $W^{1, p}(\Omega)$ for the Sobolev space of weakly differentiable functions $v: \Omega \rightarrow \mathbb{R}$ with $|v|,|D v| \in L^{p}(\Omega)$. Similarly, a function $v \in W_{\text {loc }}^{1, p}(\Omega)$ if $v \in W^{1, p}(K)$ for all compact sets $K \subset \Omega$. We denote by $W_{0}^{1, p}(\Omega)$ the closure of $C_{0}^{\infty}(\Omega)$ with respect to the norm in $W^{1, p}(\Omega)$. For any $k \in \mathbb{R}$ and a function $v \in W^{1, p}(\Omega)$, the truncations are defined by

$$
\begin{aligned}
& (v-k)_{+}=\max \{v-k, 0\} \\
& (v-k)_{-}=\max \{k-v, 0\} .
\end{aligned}
$$

Let $B$ be a Banach space and $1 \leq q<\infty$. We write $L^{q}(0, T, B)$ for the space of $L^{q}$ integrable functions from $[0, T]$ into $B$, which is a Banach space with the norm

$$
\|v\|_{L^{q}(0, T ; B)}=\left(\int_{0}^{T}\|v(t)\|_{B}^{q} \mathrm{~d} t\right)^{\frac{1}{q}} .
$$

Moreover, we write $L_{\text {loc }}^{q}(0, T, B)$ for space of functions $v \in L^{q}\left(t_{1}, t_{2}, B\right)$ for all intervals $\left[t_{1}, t_{2}\right] \subset(0, T]$. Let us denote by $C_{\text {loc }}(0, T ; B)$ the space of locally continuous functions from $[0, T]$ into $B$. Finally, we denote by $W_{p}\left(\Omega_{T}\right)$ the set of functions 


$$
v \in L_{\mathrm{loc}}^{p}\left(0, T ; W_{0}^{1, p}(\Omega)\right) \text { and } \partial_{t} v \in L_{\mathrm{loc}}^{2}\left(\Omega_{T}\right) .
$$

We are now prepared to provide the definition of the weak solution to the doubly singular parabolic equations (1.1)-(1.3):

Definition 2.1 A function $u: \Omega_{T} \rightarrow \mathbb{R}$ is said to be a local weak solution of (1.1)-(1.3) if

$$
u \in C_{\mathrm{loc}}\left(0, T ; L_{\mathrm{loc}}^{2}(\Omega)\right) \cap L_{\mathrm{loc}}^{p}\left(0, T ; W_{\mathrm{loc}}^{1, p}(\Omega)\right)
$$

and there exists a function $w \subset \beta(u)$, with the inclusion being intended in the sense of the graphs, such that the identity

$$
\left.\int_{\Omega} w(\cdot, t) \varphi(\cdot, t) \mathrm{d} x\right|_{t=t_{0}} ^{t_{1}}+\int_{t_{0}}^{t_{1}} \int_{\Omega}\left[-w \partial_{t} \varphi+A(x, t, u, D u) \cdot D \varphi\right] \mathrm{d} x \mathrm{~d} t=0
$$

holds for all testing functions $\varphi \in W_{p}\left(\Omega_{T}\right)$ and all intervals $\left[t_{0}, t_{1}\right] \subset(0, T]$.

Throughout the paper, we assume that the weak solution is bounded and that its time derivative is locally square integrable. To be more precise, we assume that

$$
\underset{\Omega_{T}}{\operatorname{ess} \sup }|u| \leq 1,
$$

and the time derivative $\partial_{t} u$ exists and satisfies

$$
\partial_{t} u \in L_{\mathrm{loc}}^{2}\left(\Omega_{T}\right) .
$$

The statement that a constant $C$ depends only upon the data means that it can be determined a priori only in terms of $\left\{\bar{v}, N, C_{0}, C_{1}\right\}$. Since we are concerned about the behavior of constants as $p \rightarrow 2$, the exponent $p$ should be excluded from the concept of the data. We can now state our main result:

Theorem 2.2 There exists a constant $\varepsilon_{0}>0$, which can be determined a priori only in terms of the data, such that the following holds: Let $u$ be a weak solution to the singular parabolic equation (1.1)-(1.3) with $p \in\left[2-\varepsilon_{0}, 2\right)$. Suppose that assumptions (2.4) and (2.5) are in force. Then, the weak solution $u$ is locally continuous, and the continuity is stable as $p \rightarrow 2$.

Finally, we collect two results regarding Sobolev inequalities that will be of use later.

Lemma 2.3 ([5, Chapter I, Proposition 2.1]) Let $\Omega \subset \mathbb{R}^{N}$ be a bounded convex set and let $\varphi \in C(\Omega)$ satisfy $0 \leq \varphi \leq 1$ and the sets $[\varphi \geq k]$ be convex for all $k \in(0,1)$. Let $v \in W^{1, p}(\Omega)$ with $1<p \leq 2$, and assume that $|[v=0] \cap[\varphi=1]|>0$. Then, there exists a constant $\gamma$ depending only upon $N$ such that

$$
\left(\int_{\Omega} \varphi|v|^{p} \mathrm{~d} x\right)^{\frac{1}{p}} \leq \gamma \frac{(\operatorname{diam} \Omega)^{N}}{|[v=0] \cap[\varphi=1]|^{\frac{N-1}{N}}}\left(\int_{\Omega} \varphi|D v|^{p} \mathrm{~d} x\right)^{\frac{1}{p}} .
$$

Lemma 2.4 ([5, Chapter I, Proposition 3.1]) Let $\Omega \subset \mathbb{R}^{N}$ be a bounded set and $N \geq 3$. Assume that $v \in L^{\infty}\left(0, T ; L^{2}(\Omega)\right) \cap L^{p}\left(0, T ; W_{0}^{1, p}(\Omega)\right)$ and $1<p \leq 2$. Then, there exists a constant $\gamma$ depending only upon $N$ such that

$$
\iint_{\Omega_{T}}|v|^{p \frac{N+2}{N}} \mathrm{~d} x \mathrm{~d} t \leq \gamma\left(\iint_{\Omega_{T}}|D v|^{p} \mathrm{~d} x \mathrm{~d} t\right)\left(\underset{0<t<T}{\operatorname{ess} \sup } \int_{\Omega}|v|^{2} \mathrm{~d} x\right)^{\frac{p}{N}} .
$$


Proof We only need to check that the constant $\gamma$ is independent of $p$. For any fixed $t \in(0, T)$, we apply [7, page $64(2.13)]$ to obtain

$$
\|v(\cdot, t)\|_{L^{N-p}(\Omega)} \leq \frac{(N-1) p}{N-p}\|D v(\cdot, t)\|_{L^{p}(\Omega)} \leq 2(N-1)\|D v(\cdot, t)\|_{L^{p}(\Omega)},
$$

since $N \geq 3$ and $1<p \leq 2$. Based on the argument of DiBenedetto [5, page 8], we obtain

$$
\begin{aligned}
\iint_{\Omega_{T}}|v|^{p \frac{N+2}{N}} \mathrm{~d} x \mathrm{~d} t & \leq \int_{0}^{T}\|v(\cdot, t)\|_{L^{\frac{N p}{N-p}}(\Omega)}^{p}\|v(\cdot, t)\|_{L^{2}(\Omega)}^{\frac{2 p}{N}} \mathrm{~d} t \\
& \leq 4(N-1)^{2}\left(\iint_{\Omega_{T}}|D v|^{p} \mathrm{~d} x \mathrm{~d} t\right)\left(\underset{0<t<T}{\left.\operatorname{ess} \sup _{\Omega}|v|^{2} \mathrm{~d} x\right)^{\frac{p}{N}},}\right.
\end{aligned}
$$

where the constant on the right-hand side is independent of $p$.

\section{Local energy estimates}

In this section, we state two energy estimates for the cutoff functions $(u-k)_{ \pm}$, where $k \in \mathbb{R}$ is a fixed number. From the assumption (2.5), the time derivative exists in the weak sense and is square integrable. This enables us to proceed similarly to the discussions in [4, page 133]. To this end, we introduce the auxiliary function

$$
v(x, t)= \begin{cases}\bar{v}, & \text { on }[u<0], \\ -w(x, t), & \text { on }[u=0],\end{cases}
$$

and the weak form (2.3) can be rewritten as

$$
\begin{gathered}
-\left.\int_{\Omega} v(\cdot, t) \chi[u \leq 0] \varphi(\cdot, t) \mathrm{d} x\right|_{t=t_{0}} ^{t_{1}}+\int_{t_{0}}^{t_{1}} \int_{\Omega} v \chi_{[u \leq 0]} \partial_{t} \varphi \mathrm{d} x \mathrm{~d} t \\
+\int_{t_{0}}^{t_{1}} \int_{\Omega}\left[\varphi \partial_{t} u+A(x, t, u, D u) \cdot D \varphi\right] \mathrm{d} x \mathrm{~d} t=0
\end{gathered}
$$

where $\varphi \in W_{p}\left(\Omega_{T}\right)$ and $\left[t_{0}, t_{1}\right] \subset(0, T]$. The appearance of the first two terms in (3.1) is due to the singularity in the time derivative, which can cause additional difficulty in the proof. Henceforth, we denote the first two terms in (3.1) by the quantity

$$
U\left(\Omega, t_{0}, t_{1}, \varphi\right)=\left.\int_{\Omega} v(\cdot, t) \chi_{[u \leq 0]} \varphi(\cdot, t) \mathrm{d} x\right|_{t=t_{0}} ^{t_{1}}-\int_{t_{0}}^{t_{1}} \int_{\Omega} v \chi_{[u \leq 0]} \partial_{t} \varphi \mathrm{d} x \mathrm{~d} t .
$$

Let $\bar{x} \in \Omega$ and $K$ be a cube centered at the origin such that $[\bar{x}+K] \subset \Omega$. We denote by $\zeta$ a piecewise smooth function in $[\bar{x}+K] \times\left[t_{0}, t_{1}\right]$ such that

$$
0 \leq \zeta \leq 1, \quad|D \zeta|<\infty \text { and } \zeta=0 \quad \text { on } \quad \partial[\bar{x}+K]
$$

By substituting $\varphi= \pm(u-k)_{ \pm} \zeta^{p}$ into (3.1), we obtain the following proposition, the proof of which we omit (see [5, Chapter II, Proposition 3.1]). 
Proposition 3.1 Let $u$ be a weak solution of (1.1)-(1.3) in $\Omega_{T}$. There exists a constant $\gamma_{1}$ that can be determined a priori only in terms of the data such that

$$
\begin{aligned}
& \underset{t_{0}<t<t_{1}}{\operatorname{ess} \sup _{[\bar{x}+K] \times\{t\}}} \int_{\leq}(u-k)_{ \pm}^{2} \zeta^{p} \mathrm{~d} x+\int_{t_{0}}^{t_{1}} \int_{[\bar{x}+K]}\left|D(u-k)_{ \pm} \zeta\right|^{p} \mathrm{~d} x \mathrm{~d} t \\
& \leq \int_{[\bar{x}+K] \times\left\{t_{0}\right\}}(u-k)_{ \pm}^{2} \zeta^{p} \mathrm{~d} x+\gamma_{1} \int_{t_{0}}^{t_{1}} \int_{[\bar{x}+K]}(u-k)_{ \pm}^{p}|D \zeta|^{p} \mathrm{~d} x \mathrm{~d} t \\
& \quad+\gamma_{1} \int_{t_{0}}^{t_{1}} \int_{[\bar{x}+K]}(u-k)_{ \pm}^{2}\left|\partial_{t} \zeta\right| \mathrm{d} x \mathrm{~d} t+U\left([\bar{x}+K], t_{0}, t_{1}, \pm(u-k)_{ \pm} \zeta^{p}\right)
\end{aligned}
$$

We now turn to consider the local logarithmic estimates for weak solutions. To this end, we introduce the logarithmic function

$$
\psi^{ \pm}(u)=\max \left\{\ln \frac{H_{k}^{ \pm}}{H_{k}^{ \pm}-(u-k)_{ \pm}+c} ; 0\right\}, \quad 0<c<H_{k}^{ \pm},
$$

where $H_{k}^{ \pm}$is a constant chosen such that

$$
H_{k}^{ \pm} \geq \operatorname{ess~sup}_{[\bar{x}+K] \times\left[t_{0}, t_{1}\right]}(u-k)_{ \pm} .
$$

For simplicity of notation, we write $\psi^{ \pm}$instead of $\psi^{ \pm}(u)$. We let $\left(\psi^{ \pm}\right)^{\prime}$ stand for the derivative of $\psi^{ \pm}(u)$ with respect to $u$. If we plug $\varphi=2 \psi^{ \pm}\left(\psi^{ \pm}\right)^{\prime} \zeta^{p}$ into (3.1), we obtain the following proposition.

Proposition 3.2 Let $u$ be a weak solution of (1.1)-(1.3) in $\Omega_{T}$. There exists a constant $\gamma_{2}$ that can be determined a priori only in terms of the data such that

$$
\begin{aligned}
& \underset{t_{0}<t<t_{1}}{\operatorname{ess~sup}} \int_{[\bar{x}+K] \times\{t\}}\left(\psi^{ \pm}\right)^{2} \zeta^{p} \mathrm{~d} x \leq \int_{[\bar{x}+K] \times\left\{t_{0}\right\}}\left(\psi^{ \pm}\right)^{2} \zeta^{p} \mathrm{~d} x \\
& \quad+\gamma_{2} \int_{t_{0}}^{t_{1}} \int_{[\bar{x}+K]} \psi^{ \pm}\left|\left(\psi^{ \pm}\right)^{\prime}\right|^{2-p}|D \zeta|^{p} \mathrm{~d} x \mathrm{~d} t+U\left([\bar{x}+K], t_{0}, t_{1}, 2 \psi^{ \pm}\left(\psi^{ \pm}\right)^{\prime} \zeta^{p}\right),
\end{aligned}
$$

where $\zeta=\zeta(x)$ is independent of $t \in\left[t_{0}, t_{1}\right]$ and satisfies (3.2).

This is a standard result that can be found in [5, Chapter II, Proposition 3.2] and no proof will be given here. Finally, we remark that our approach does not follow the idea in [6] and [10], which concerns the approximate solutions to the equations with regularization of the maximal monotone graph. Instead, we follow the approach in [4], which is more convenient for addressing the second alternative.

\section{The intrinsic geometry}

The continuity of $u$ will be a consequence of the following assertion. For any point $\left(x_{0}, t_{0}\right) \in$ $\Omega_{T}$, there exists a family of nested and shrinking cylinders $\left[\left(x_{0}, t_{0}\right)+Q_{n}\right]$ such that the oscillation of $u$ in $\left[\left(x_{0}, t_{0}\right)+Q_{n}\right]$ tends to zero as $n \rightarrow \infty$. To begin the proof, we introduce a certain intrinsic parabolic cylinder that plays an important role in alternative arguments.

Without loss of generality, we assume that $\left(x_{0}, t_{0}\right)=(0,0)$. Let $R<1$ be a fixed number such that $Q\left(R, R^{\frac{1}{p}}\right) \subset \Omega_{T}$, and we set $\bar{Q}=Q\left(R, R^{\frac{1}{p}}\right)$ as a reference parabolic cylinder. We write

$$
\mu_{-}=\underset{\bar{Q}}{\operatorname{essinf}} u, \quad \mu_{+}=\underset{\bar{Q}}{\operatorname{ess} \sup u} \text { and } \quad \underset{\bar{Q}}{\operatorname{ess} \operatorname{osc}} u=\mu_{+}-\mu_{-} .
$$


Here and subsequently, $\omega$ stands for a fixed number and satisfies $\omega>\operatorname{ess} \operatorname{osc} u$. For $(\bar{x}, \bar{t}) \in$ $\bar{Q}$, we introduce the intrinsic parabolic cylinders of the form $(\bar{x}, \bar{t})+Q\left(\bar{Q} R^{p}, d_{*} R\right)$ where

$$
d=\left(\frac{\omega}{2^{m_{1}}}\right)^{(1-p)(2-p)}, \quad d_{*}=\left(\frac{\omega}{2^{m_{2}}}\right)^{p-2} \text { and } m_{1}=\frac{p}{p-1} m_{2} .
$$

The quantities $m_{1}$ and $m_{2}$ depend upon $\omega$ and will be determined in Sect. 6.2. Moreover, the relation $m_{1}=p m_{2} /(p-1)$ implies

$$
\frac{d_{*}^{p}}{d}=\frac{\left(\frac{\omega}{2^{m} 2}\right)^{p(p-2)}}{\left(\frac{\omega}{2^{m} 1}\right)^{(1-p)(2-p)}}=\omega^{p-2} .
$$

Let $L_{1}>1$ and $L_{2}>1$ denote the fixed constants, which will be determined in Sect. 5 . We first assume that $Q\left(c_{1} R^{p}, c_{2} R\right) \subset \bar{Q}$, where $c_{1}=L_{1} d$ and $c_{2}=L_{2} d_{*}$. In the case $Q\left(c_{1} R^{p}, c_{2} R\right) \nsubseteq \bar{Q}$, we shall derive a decay estimate for $\omega$ in terms of $R$, and the discussion is postponed until Sect. 6.2. Define $K_{\omega}=K_{\left(L_{2}-1\right) d_{*} R}$ and $I_{\omega}=\left[-\left(L_{1}-1\right) d R^{p}, 0\right]$. It is easy to check that $K_{\frac{1}{2} c_{2} R} \subset K_{\omega}$.

The main ingredient of the proof is to establish an estimate for the essential oscillation of $u$ in a smaller cylinder $Q\left(d\left(\frac{R}{2}\right)^{p}, d_{*}\left(\frac{R}{2}\right)\right)$,

$$
\underset{Q\left(d\left(\frac{R}{2}\right)^{p}, d_{*}\left(\frac{R}{2}\right)\right)}{\operatorname{ess} \text { osc }} u \leq \sigma(\omega) \omega
$$

where $\sigma(\omega)<1$. We now assume that $\mu_{+}-\mu_{-} \geq \omega / 2$; otherwise, (4.3) follows immediately. Moreover, for technical reasons, we must separately consider the two cases $\mu_{+} \geq\left|\mu_{-}\right|$and $\mu_{+}<\left|\mu_{-}\right|$.

Motivated by the work of Henriques and Urbano [6], we consider the four complementary cases described below. Let $\mu_{+} \geq\left|\mu_{-}\right|$. In Sect. 5 we shall determine a constant $v_{0} \in(0,1)$ and we need to distinguish two possible alternatives.

The first alternative There exists $\bar{t} \in I(\omega)$ such that for all $\bar{x} \in K_{\omega}$,

$$
\left|\left\{(x, t) \in(\bar{x}, \bar{t})+Q\left(d R^{p}, d_{*} R\right): u<\mu_{-}+\frac{\omega}{4}\right\}\right| \leq v_{0}\left|Q\left(d R^{p}, d_{*} R\right)\right| .
$$

The second alternative For any $\bar{t} \in I(\omega)$, there exists $\bar{x} \in K_{\omega}$ such that

$$
\left|\left\{(x, t) \in(\bar{x}, \bar{t})+Q\left(d R^{p}, d_{*} R\right): u>\mu_{+}-\frac{\omega}{4}\right\}\right| \leq\left(1-v_{0}\right)\left|Q\left(d R^{p}, d_{*} R\right)\right| .
$$

Similarly, if $\mu_{+}<\left|\mu_{-}\right|$we must distinguish two alternatives, described by a constant $\nu_{0} \in(0,1)$ which can be determined by $\omega$.

The third alternative There exists $\bar{t} \in I(\omega)$ such that for all $\bar{x} \in K_{\omega}$,

$$
\left|\left\{(x, t) \in(\bar{x}, \bar{t})+Q\left(d R^{p}, d_{*} R\right): u>\mu_{+}-\frac{\omega}{4}\right\}\right| \leq v_{0}\left|Q\left(d R^{p}, d_{*} R\right)\right| .
$$

The fourth alternative For any $\bar{t} \in I(\omega)$, there exists $\bar{x} \in K_{\omega}$ such that

$$
\left|\left\{(x, t) \in(\bar{x}, \bar{t})+Q\left(d R^{p}, d_{*} R\right): u<\mu_{-}+\frac{\omega}{4}\right\}\right| \leq\left(1-v_{0}\right)\left|Q\left(d R^{p}, d_{*} R\right)\right| .
$$

For simplicity, we concentrate in the next two sections only the case $\mu_{+} \geq\left|\mu_{-}\right|$, since the treatment of the case $\mu_{+}<\left|\mu_{-}\right|$is similar. The proof of (4.3) in the case $\mu_{+}<\left|\mu_{-}\right|$is left to the reader. 


\section{The first alternative}

The aim of this section is to establish the estimate (4.3) for the first alternative. First, we establish the following DeGiorgi-type lemma and determine the constant $v_{0}$ in terms of the data and $\omega$.

Lemma 5.1 There exists a constant $v_{0} \in(0,1)$, depending only upon data and $\omega$, such that if (4.4) holds for some $(\bar{x}, \bar{t}) \in K_{\omega} \times I_{\omega}$, then

$$
u(x, t) \geq \mu_{-}+\frac{\omega}{8} \text { a.e. } \quad(x, t) \in(\bar{x}, \bar{t})+Q\left(d\left(\frac{R}{2}\right)^{p}, d_{*} \frac{R}{2}\right) .
$$

Proof Without loss of generality, we may assume that $(\bar{x}, \bar{t})=(0,0)$. Define two decreasing sequences of numbers

$$
R_{n}=\frac{R}{2}+\frac{R}{2^{n+1}}, \quad k_{n}=\mu_{-}+\frac{\omega}{8}+\frac{\omega}{2^{n+3}}, \quad n=0,1,2, \ldots,
$$

and construct the family of nested and shrinking cylinders $Q_{n}=Q\left(d R_{n}^{p}, d_{*} R_{n}\right)$. We construct smooth cutoff functions $0 \leq \zeta_{n} \leq 1$, such that $\zeta_{n} \equiv 1$ in $Q_{n+1}, \zeta_{n} \equiv 0$ on $\partial_{P} Q_{n}$, $\left|D \zeta_{n}\right| \leq 2^{n+2} /\left(d_{*} R\right)$ and $0<\partial_{t} \zeta_{n} \leq 2^{2+n p} /\left(d R^{p}\right)$. Write the energy estimate (3.3) over the cylinder $Q_{n}$ for the functions $\left(u-k_{n}\right)_{-}$. We first observe from [4, page 145] that

$$
\begin{aligned}
& U\left(K_{d_{*} R_{n}},-d R_{n}^{p}, 0,-\left(u-k_{n}\right)_{-} \zeta_{n}^{p}\right) \leq \bar{v} \iint_{Q_{n}}\left(u-k_{n}\right)_{-} \partial_{t} \zeta_{n}^{p} \mathrm{~d} x \mathrm{~d} t \\
& \quad \leq 2 \bar{v} \iint_{Q_{n}}\left(u-k_{n}\right)_{-} \partial_{t} \zeta_{n} \mathrm{~d} x \mathrm{~d} t .
\end{aligned}
$$

Since $\left(u-k_{n}\right)_{-} \leq \frac{\omega}{4}<1$ and $\partial_{t} \zeta_{n} \geq 0$, we obtain

$$
\begin{aligned}
& \underset{-d R_{n}^{p}<t<0}{\operatorname{ess} \sup } \int_{K_{d_{*} R_{n}} \times\{t\}}\left(u-k_{n}\right)_{-}^{2} \zeta_{n}^{p} \mathrm{~d} x+\iint_{Q_{n}}\left|D\left(u-k_{n}\right)_{-} \zeta_{n}\right|^{p} \mathrm{~d} x \mathrm{~d} t \\
& \leq \gamma_{1} \iint_{Q_{n}}\left(u-k_{n}\right)_{-}^{p}\left|D \zeta_{n}\right|^{p} \mathrm{~d} x \mathrm{~d} t+(1+2 \bar{v}) \gamma_{1} \iint_{Q_{n}}\left(u-k_{n}\right)_{-} \partial_{t} \zeta_{n} \mathrm{~d} x \mathrm{~d} t \\
& \leq \bar{\gamma}\left(\left(\frac{\omega}{4}\right)^{p} \frac{2^{2 n}}{\left(d_{*} R\right)^{p}}+\left(\frac{\omega}{4}\right) \frac{2^{2 n}}{d R^{p}}\right) \iint_{Q_{n}} \chi_{\left[\left(u-k_{n}\right)_{-}>0\right]} \mathrm{d} x \mathrm{~d} t,
\end{aligned}
$$

where $\bar{\gamma}=(1+2 \bar{v}) \gamma_{1}$. Moreover, the relation (4.2) implies

$$
\left(\frac{\omega}{4}\right)^{p} \frac{2^{2 n}}{\left(d_{*} R\right)^{p}}=\frac{1}{4^{p}} \frac{\omega^{2} 2^{2 n}}{d R^{p}} \leq\left(\frac{\omega}{4}\right) \frac{2^{2 n}}{d R^{p}},
$$

since $\omega<1$. Then, we conclude that

$$
\begin{aligned}
& \underset{-d R_{n}^{p}<t<0}{\operatorname{ess} \sup } \int_{K_{d_{*} R_{n}} \times\{t\}}\left(u-k_{n}\right)_{-}^{2} \zeta_{n}^{p} \mathrm{~d} x+\iint_{Q_{n}}\left|D\left(u-k_{n}\right)_{-} \zeta_{n}\right|^{p} \mathrm{~d} x \mathrm{~d} t \\
& \quad \leq \bar{\gamma}\left(\frac{\omega}{4}\right) \frac{2^{2 n}}{d R^{p}} \iint_{Q_{n}} \chi_{\left[\left(u-k_{n}\right)_{-}>0\right]} \mathrm{d} x \mathrm{~d} t .
\end{aligned}
$$


Applying the parabolic Sobolev inequality (2.7), we deduce

$$
\begin{aligned}
& \iint_{Q_{n}}\left|\left(u-k_{n}\right)_{-} \zeta\right|^{p \frac{N+2}{N}} \mathrm{~d} x \mathrm{~d} t \\
& \quad \leq \gamma\left(\underset{-d R_{n}^{p}<t<0}{\operatorname{ess} \sup } \int_{K_{d_{*} R_{n}} \times\{t\}}\left(u-k_{n}\right)_{-}^{2} \zeta_{n}^{2} \mathrm{~d} x\right)^{\frac{p}{N}} \\
& \quad \iint_{Q_{n}}\left|D\left(u-k_{n}\right)_{-} \zeta_{n}\right|^{p} \mathrm{~d} x \mathrm{~d} t \\
& \quad \leq \gamma \bar{\gamma}\left(\frac{\omega}{4}\right)^{1+\frac{p}{N}}\left(\frac{2^{2 n}}{d R^{p}} \iint_{Q_{n}} \chi_{\left[\left(u-k_{n}\right)_{->0}\right]} \mathrm{d} x \mathrm{~d} t\right)^{1+\frac{p}{N}} .
\end{aligned}
$$

At this point, we set

$$
A_{n}=\iint_{Q_{n}} \chi_{\left[\left(u-k_{n}\right)_{->0}\right.} \mathrm{d} x \mathrm{~d} t \text { and } Y_{n}=\frac{A_{n}}{\left|Q_{n}\right|} .
$$

The left-hand side of (5.2) is estimated below by

$$
\begin{gathered}
\iint_{Q_{n}}\left|\left(u-k_{n}\right)_{-} \zeta\right|^{p \frac{N+2}{N}} \mathrm{~d} x \mathrm{~d} t \geq \iint_{Q_{n+1}}\left|\left(u-k_{n}\right)_{-}\right|^{p \frac{N+2}{N}} \chi_{\left[\left(u-k_{n+1}\right)_{-}>0\right]} \mathrm{d} x \mathrm{~d} t \\
\geq\left(k_{n}-k_{n+1}\right)^{p \frac{N+2}{N}} A_{n+1} \geq\left(\frac{\omega}{2^{n+4}}\right)^{p \frac{N+2}{N}} A_{n+1} .
\end{gathered}
$$

Combining (5.3) with (5.2) and noting that $\left|Q_{n}\right|=d d_{*}^{N} R_{n}^{N+p}$, we obtain

$$
Y_{n+1} \leq \gamma \bar{\gamma} 2^{10+N+\frac{16}{N}} \frac{\omega^{1-p-\frac{p}{N}} 2^{\left(4+\frac{8}{N}\right) n}}{d d_{*}^{-p}} Y_{n}^{1+\frac{p}{N}}=\gamma \bar{\gamma} 2^{10+N+\frac{16}{N}} \omega^{-1-\frac{p}{N}} 2^{\left(4+\frac{8}{N}\right) n} Y_{n}^{1+\frac{p}{N}},
$$

where we used (4.2) in the last step. Next, we set

$$
v_{0}=\left(\gamma \bar{\gamma} 2^{10+N+\frac{16}{N}}\right)^{-N} 2^{-N^{2}\left(4+\frac{8}{N}\right)} \omega^{N+1} .
$$

We observe that if $Y_{0} \leq v_{0}$, then

$$
Y_{0} \leq\left(\gamma \bar{\gamma} 2^{10+N+\frac{16}{N}} \omega^{-1-\frac{p}{N}} \omega^{-1-\frac{p}{N}}\right)^{-\frac{N}{p}} 2^{-\frac{N^{2}}{p^{2}}\left(4+\frac{8}{N}\right)} .
$$

Using a lemma on fast geometric convergence of sequences (cf. [5, Chapter I, Lemma 4.1]), we conclude that $Y_{n} \rightarrow 0$ as $n \rightarrow \infty$. This completes the proof of Lemma 5.1.

From the definition of the first alternative, we can extend the estimate (5.1) to a larger domain by using a covering argument. To be more precise, we obtain the following corollary, the proof of which we omit.

Corollary 5.2 Let $v_{0}$ be a constant chosen according to (5.4), and assume that (4.4) holds for some $\bar{t} \in I_{\omega}$, and for all $\bar{x} \in K_{\omega}$. Then, there holds

$$
u(x, t) \geq \mu_{-}+\frac{\omega}{8} \text { a.e. }(x, t) \in K_{c_{2} \frac{R}{2}} \times\left(\bar{t}-d\left(\frac{R}{2}\right)^{p}, \bar{t}\right] .
$$

Next, we set $t_{*}=\bar{t}-d(R / 2)^{p}$ and establish the following result regarding the expansion of the positivity in the time direction. 
Lemma 5.3 Let $v_{0}$ be a constant chosen according to (5.4), and assume that (4.4) holds for some $\bar{t} \in I_{\omega}$ and for all $\bar{x} \in K_{\omega}$. For any fixed $\nu_{1} \in(0,1)$, the constant $L_{2}$ can be chosen in dependence on $N, C_{0}, C_{1}, v_{1}$ and $L_{1}$ such that, with $c_{2}=L_{2} d_{*}$, there holds

$$
\left|\left\{x \in K_{c_{2} \frac{R}{4}}: u<\mu_{-}+\frac{\omega}{2^{6}}\right\}\right|<v_{1}\left|K_{c_{2} \frac{R}{4}}\right|
$$

for all $t \in\left(t_{*}, 0\right)$.

Proof We first recall that

$$
\begin{aligned}
\psi^{-} & =\ln ^{+}\left(\frac{H_{k}^{-}}{H_{k}^{-}-(u-k)_{-}+c}\right) \\
& = \begin{cases}\ln \left(\frac{H_{k}^{-}}{H_{k}^{-}+u-k+c}\right), & k-H_{k}^{-} \leq u<k-c, \\
0, & u \geq k-c,\end{cases}
\end{aligned}
$$

and

$$
\left(\psi^{-}\right)^{\prime}= \begin{cases}-\frac{1}{H_{k}^{-}+u-k+c}, & k-H_{k}^{-} \leq u<k-c, \\ 0, & u \geq k-c,\end{cases}
$$

where

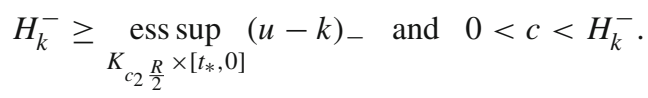

Next, we let $k=\mu_{-}+\frac{\omega}{8}$ and choose $H_{k}^{-}=\frac{\omega}{8}$, which is admissible since $(u-k)_{-} \leq \frac{\omega}{8}$. We observe from (5.5) that $\psi^{-}\left(x, t_{*}\right)=0$ for all $x \in K_{c_{2}} \frac{R}{2}$. The following proof will be divided into two steps.

Step 1: Let $\zeta \equiv \zeta(x)$ be a piecewise smooth function. We establish the estimate:

$$
U\left(K_{c_{2} \frac{R}{2}}, t_{*}, 0,2 \psi^{-}\left(\psi^{-}\right)^{\prime} \zeta^{p}\right) \leq 0 .
$$

In the case $k \leq 0$, we have $u<k-c<0$ and this yields

$$
\begin{aligned}
& U\left(K_{c_{2} \frac{R}{2}}, t_{*}, 0,2 \psi^{-}\left(\psi^{-}\right)^{\prime} \zeta^{p}\right) \\
& \quad=\left.2 \bar{v} \int_{K_{c_{2} \frac{R}{2}}} \psi^{-}\left(\psi^{-}\right)^{\prime} \zeta^{p} \mathrm{~d} x\right|_{t=t_{*}} ^{0}-2 \bar{v} \int_{t_{*}}^{0} \int_{K_{c_{2} \frac{R}{2}}} \frac{\partial\left[\psi^{-}\left(\psi^{-}\right)^{\prime} \zeta^{p}\right]}{\partial t} \mathrm{~d} x \mathrm{~d} t=0,
\end{aligned}
$$

which is our claim. We now turn to the case $k>0$. If $\mu_{-} \geq c$, then there holds $u \geq \mu_{-} \geq$ $c>0$, and we again obtain the identity $U\left(K_{c_{2} \frac{R}{2}}, t_{*}, 0,2 \psi^{-}\left(\psi^{-}\right)^{\prime} \zeta^{p}\right)=0$. It now remains to consider the case $\mu_{-}<c$. Since $\zeta \equiv \zeta(x)$, then $\partial_{t} \zeta \equiv 0$. We write 


$$
\begin{aligned}
U( & \left.K_{c_{2} \frac{R}{2}}, t_{*}, 0,2 \psi^{-}\left(\psi^{-}\right)^{\prime} \zeta^{p}\right) \\
= & \left.2 \int_{K_{c_{2} \frac{R}{2}}} v(\cdot, t) \chi_{[u \leq 0]} \psi^{-}\left(\psi^{-}\right)^{\prime} \zeta^{p} \mathrm{~d} x\right|_{t=t_{*}} ^{0} \\
& -2 \int_{t_{*}}^{0} \int_{K_{c_{2} \frac{R}{2}}} v \chi_{[u \leq 0]} \frac{\partial\left[\psi^{-}\left(\psi^{-}\right)^{\prime}\right]}{\partial t} \zeta^{p} \mathrm{~d} x \mathrm{~d} t \\
= & : S_{1}+S_{2},
\end{aligned}
$$

with the obvious meaning of $S_{1}$ and $S_{2}$. We begin with the estimate for $S_{1}$. Noting that $-\left(\psi^{-}\right)^{\prime}=\left(u-\mu_{-}+c\right)^{-1} \leq c^{-1}$, we obtain

$$
\begin{aligned}
S_{1}= & \left.2 \int_{K_{c_{2} \frac{R}{2}}} v(\cdot, t) \chi_{[u \leq 0]} \psi^{-}\left(\psi^{-}\right)^{\prime} \zeta^{p} \mathrm{~d} x\right|_{t=0} \\
& +\left.\left.2 \int_{K_{c_{2} \frac{R}{2}}} v(\cdot, t) \chi_{[u \leq 0]} \psi^{-}\left(-\left(\psi^{-}\right)^{\prime}\right) \zeta^{p} \mathrm{~d} x\right|_{t=t_{*}} \psi^{-}\left(\psi^{-}\right)^{\prime} \zeta^{p} \mathrm{~d} x\right|_{t=0}+\left.\frac{2 \bar{v}}{c} \int_{K_{c_{2} \frac{R}{2}}} \psi^{-} \zeta^{p} \mathrm{~d} x\right|_{t=t_{*}} .
\end{aligned}
$$

To estimate $S_{2}$, we note that $\partial u / \partial t \equiv 0$ on $[u=0]$ and there holds

$$
\begin{aligned}
S_{2} & =2 \bar{v} \int_{t_{*}}^{0} \int_{K_{c_{2} \frac{R}{2}}}\left[\psi^{-}\left(\psi^{-}\right)^{\prime}\right]^{\prime} \frac{\partial u_{-}}{\partial t} \zeta^{p} \mathrm{~d} x \mathrm{~d} t \\
& =-2 \bar{v} \int_{t_{*}}^{0} \int_{K_{c_{2} \frac{R}{2}}} \chi_{[u<0]} \frac{\partial\left[\psi^{-}\left(\psi^{-}\right)^{\prime}\right]_{\zeta^{2}}}{\partial t} \mathrm{~d} x \mathrm{~d} t .
\end{aligned}
$$

At this stage, we introduce an auxiliary function $\Phi_{c}(u)$, defined by

$$
\begin{aligned}
\Phi_{c}(u) & = \begin{cases}-\psi^{-}(u)\left(\psi^{-}\right)^{\prime}(u)+\psi^{-}(0)\left(\psi^{-}\right)^{\prime}(0), & \mu_{-} \leq u<0, \\
0, & 0 \leq u<\mu_{-}+\frac{\omega}{8}-c,\end{cases} \\
& = \begin{cases}\ln \left(\frac{\omega}{8\left(u-\mu_{-}+c\right)}\right) \frac{1}{u-\mu_{-}+c}-\ln \left(\frac{\omega}{8\left(c-\mu_{-}\right)}\right) \frac{1}{c-\mu_{-}}, & \mu_{-} \leq u<0, \\
0, & 0 \leq u<\mu_{-}+\frac{\omega}{8}-c .\end{cases}
\end{aligned}
$$

We observe that $0 \leq \Phi_{c}(u) \leq-\psi^{-}\left(\psi^{-}\right)^{\prime}, \Phi_{c}(u)$ is Lipschitz with respect to $u$ and

$$
\frac{\partial \Phi_{c}(u)}{\partial t}=-\frac{\partial\left[\psi^{-}\left(\psi^{-}\right)^{\prime}\right]}{\partial t} \chi_{[u<0]} .
$$

Integrating by parts, we deduce

$$
\begin{aligned}
S_{2} & =2 \bar{v} \int_{t_{*}}^{0} \int_{K_{c_{2} \frac{R}{2}}} \frac{\partial \Phi_{c}}{\partial t} \zeta^{p} \mathrm{~d} x \mathrm{~d} t \\
& =\left.2 \bar{v} \int_{K_{c_{2} \frac{R}{2}}} \Phi_{c} \zeta^{p} \mathrm{~d} x\right|_{t=0}-\left.2 \bar{v} \int_{K_{c_{2} \frac{R}{2}}} \Phi_{c} \zeta^{p} \mathrm{~d} x\right|_{t=t_{*}}
\end{aligned}
$$




$$
\begin{aligned}
& \leq\left. 2 \bar{v} \int_{K_{c_{2} \frac{R}{2}}} \Phi_{c} \zeta^{p} \mathrm{~d} x\right|_{t=0} \\
& \leq-\left.2 \bar{v} \int_{K_{c_{2} \frac{R}{2}} \cap[u<0]} \psi^{-}\left(\psi^{-}\right)^{\prime} \zeta^{p} \mathrm{~d} x\right|_{t=0} .
\end{aligned}
$$

Combining (5.10) and (5.11), we conclude that

$$
U\left(K_{c_{2} \frac{R}{2}}, t_{*}, 0,2 \psi^{-}\left(\psi^{-}\right)^{\prime} \zeta^{p}\right)=S_{1}+S_{2} \leq\left.\frac{2 \bar{v}}{c} \int_{K_{c_{2} \frac{R}{2}}} \psi^{-} \zeta^{p} \mathrm{~d} x\right|_{t=t_{*}}=0,
$$

since $\psi^{-}\left(x, t_{*}\right)=0$ for all $x \in K_{c_{2} \frac{R}{2}}$. This proves the claim (5.9).

Step 2: Proof of (5.6). Since $\psi^{-}\left(x, t_{*}\right)=0$ for all $x \in K_{c_{2} \frac{R}{2}}$, we find that

$$
\int_{K_{c_{2} \frac{R}{2}} \times\left\{-t_{*}\right\}}\left(\psi^{-}\right)^{2} \zeta^{p} \mathrm{~d} x=0 .
$$

Plugging this into (3.4) and taking into account (5.9), we obtain

$$
\underset{t_{*}<t<0}{\operatorname{ess} \sup } \int_{K_{c_{2} \frac{R}{2}} \times\{t\}}\left(\psi^{-}\right)^{2} \zeta^{p} \mathrm{~d} x \leq \gamma_{2} \iint_{Q\left(\left|t_{*}\right|, c_{2} \frac{R}{2}\right)} \psi^{-}\left|\left(\psi^{-}\right)^{\prime}\right|^{2-p}|D \zeta|^{p} \mathrm{~d} x \mathrm{~d} t .
$$

We take the cutoff function $\zeta=\zeta(x)$, which satisfies $0 \leq \zeta \leq 1$ in $K_{c_{2} \frac{R}{2}}, \zeta \equiv 1$ in $K_{c_{2} \frac{R}{4}}$ and $|D \zeta| \leq 4\left(c_{2} R\right)^{-1}$. Now we take $c=2^{-6} \omega$ and deduce from (5.7) and (5.8) the estimate

$$
\psi^{-}\left|\left(\psi^{-}\right)^{\prime}\right|^{2-p}|D \zeta|^{p} \leq\left(\ln \frac{\omega}{8 c}\right) c^{p-2}\left(\frac{4}{c_{2} R}\right)^{p}=2^{11}(\ln 2) \omega^{p-2} L_{2}^{-p} d_{*}^{-p} R^{-p} .
$$

Recalling $\left|t_{*}\right| \leq L_{1} d R^{p}$, we deduce from (4.2) the estimate

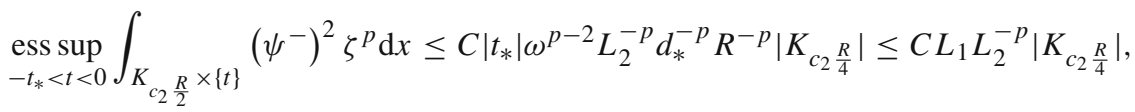

where $C=2^{N+12} \gamma_{2}>1$. At this point, we choose

$$
L_{2} \geq\left(2^{N+12} \gamma_{2}\right)^{\frac{1}{p}} \nu_{1}^{-\frac{1}{p}} L_{1}^{\frac{1}{p}}
$$

For such a choice of $L_{2}$, the above estimate yields

$$
\underset{t_{*}<t<0}{\operatorname{ess} \sup } \int_{K_{c_{2} \frac{R}{2}} \times\{t\}}\left(\psi^{-}\right)^{2} \zeta^{p} \mathrm{~d} x \leq v_{1}\left|K_{c_{2} \frac{R}{4}}\right| .
$$

The left-hand side of (5.12) is estimated below by integrating over the smaller set

$$
\left\{x \in K_{c_{2} \frac{R}{4}}: u<\mu_{-}+\frac{\omega}{2^{6}}\right\} \subset K_{c_{2} \frac{R}{2}} .
$$

On such a set, $\zeta \equiv 1$ and

$$
\psi^{-}=\ln ^{+}\left(\frac{\frac{\omega}{8}}{\frac{\omega}{8}-(u-k)_{-}+\frac{\omega}{2^{6}}}\right) \geq 2 \ln 2>1 .
$$


This yields

$$
\int_{K_{c_{2} \frac{R}{2}} \times\{t\}}\left(\psi^{-}\right)^{2} \zeta^{p} \mathrm{~d} x \geq\left|\left\{x \in K_{c_{2} \frac{R}{4}}: u<\mu_{-}+\frac{\omega}{2^{6}}\right\}\right|,
$$

for all $t \in\left(t_{*}, 0\right)$. Combining (5.13) with (5.12), we conclude that for any $t \in\left(t_{*}, 0\right)$, there holds

$$
\left|\left\{x \in K_{c_{2} \frac{R}{4}}: u<\mu_{-}+\frac{\omega}{2^{6}}\right\}\right| \leq v_{1}\left|K_{c_{2} \frac{R}{4}}\right|,
$$

which proves the lemma.

With the help of the preceding two lemmas, we can now prove the following proposition, which is the main result in this section.

Proposition 5.4 Suppose that $Q\left(c_{1} R^{p}, c_{2} R\right) \subset \bar{Q} \subset \Omega_{T}$. Let $v_{0}$ be a constant chosen according to (5.4), and assume that (4.4) holds for some $\bar{t} \in I_{\omega}$ and for all $\bar{x} \in K_{\omega}$. Then there exists a constant $v_{1} \in(0,1)$ depending only upon the data such that the following holds. For the constant $L_{2}>0$, which is determined a priori only in terms of $\left\{N, C_{0}, C_{1}, L_{1}\right\}$ such that

$$
L_{2} \geq\left(2^{N+12} \gamma_{2}\right)^{\frac{1}{p}} v_{1}^{-\frac{1}{p}} L_{1}^{\frac{1}{p}}
$$

and $c_{2}=L_{2} d_{*}$, there holds

$$
u(x, t)>\mu_{-}+\frac{\omega}{2^{7}} \text { a.e. in } Q\left(-t_{*}, c_{2} \frac{R}{8}\right) .
$$

Proof Let $\gamma$ and $\gamma_{1}$ be the constants determined by Lemma 2.4 and 3.3, respectively, depending only upon the data $\left\{N, C_{0}, C_{1}\right\}$. We now choose

$$
v_{1}=2^{-3 N^{2}\left(1+\frac{2}{N}\right)} 2^{-22(N+2)-N^{2}} \gamma^{-N} \gamma_{1}^{-N-2}
$$

and choose $L_{2}$ satisfying (5.14). By Lemma 5.3, we conclude from (5.6) that

$$
\int_{t_{*}}^{0}\left|\left\{x \in K_{c_{2} \frac{R}{4}}: u<\mu_{-}+\frac{\omega}{2^{6}}\right\}\right| \mathrm{d} t<v_{1}\left|K_{c_{2} \frac{R}{4}} \times\left(t_{*}, 0\right)\right| .
$$

Next, we define two decreasing sequences of numbers

$$
R_{n}=\frac{R}{8}+\frac{R}{2^{n+3}}, \quad k_{n}=\mu_{-}+\frac{\omega}{2^{7}}+\frac{\omega}{2^{n+7}}, \quad n=0,1,2, \ldots
$$

We set $Q_{n}=Q\left(-t_{*}, c_{2} R_{n}\right)$ and choose piecewise smooth cutoff functions $\zeta_{n}(x)$ defined in $K_{c_{2} R_{n}}$ and satisfying $0 \leq \zeta_{n} \leq 1$ in $K_{c_{2} R_{n}}, \zeta_{n} \equiv 1$ in $K_{c_{2} R_{n+1}}$ and $\left|D \zeta_{n}\right| \leq 2^{n+4}\left(c_{2} R\right)^{-1}$. We recall that from (5.1) there holds $\left(u-k_{n}\right)_{-}\left(\cdot, t_{*}\right)=0$ in $K_{c_{2} R_{n}}$. Writing the energy estimate (3.3) for $\left(u-k_{n}\right)_{-}$over the cylinder $Q_{n}$, we obtain

$$
\begin{aligned}
& \underset{t_{*}<t<0}{\operatorname{ess} \sup } \int_{K_{c_{2} R_{n}} \times\{t\}}\left(u-k_{n}\right)_{-}^{2} \zeta_{n}^{p} \mathrm{~d} x+\iint_{Q_{n}}\left|D\left(u-k_{n}\right)_{-} \zeta_{n}\right|^{p} \mathrm{~d} x \mathrm{~d} t \\
& \leq \gamma_{1} \iint_{Q_{n}}\left(u-k_{n}\right)_{-}^{p}\left|D \zeta_{n}\right|^{p} \mathrm{~d} x \mathrm{~d} t+U\left(K_{c_{2} R_{n}}, t_{*}, 0,-\left(u-k_{n}\right)_{-} \zeta_{n}^{p}\right),
\end{aligned}
$$

since $\partial_{t} \zeta_{n} \equiv 0$. Next, we claim that

$$
U\left(K_{c_{2} R_{n}}, t_{*}, 0,-\left(u-k_{n}\right)_{-} \zeta_{n}^{p}\right) \leq 0
$$


To prove (5.18), we first consider the case $k_{n}<0$. In this case, either $\left(u-k_{n}\right)_{-}=0$ or $u \leq k_{n}<0$. We obtain

$$
\begin{aligned}
U & \left(K_{c_{2} R_{n}}, t_{*}, 0,-\left(u-k_{n}\right)_{-} \zeta_{n}^{p}\right) \\
& =-\left.\bar{v} \int_{K_{c_{2} R_{n}}}\left(u-k_{n}\right)_{-} \zeta_{n}^{p} \mathrm{~d} x\right|_{t=t_{*}} ^{0}+\bar{v} \int_{t_{*}}^{0} \int_{K_{c_{2} R_{n}}} \frac{\partial\left(u-k_{n}\right)_{-}}{\partial t} \zeta_{n}^{p} \mathrm{~d} x \mathrm{~d} t \\
& =0 .
\end{aligned}
$$

In the case $k_{n} \geq 0$, we note that $\left(u-k_{n}\right)_{-} \chi_{[u \leq 0]}=k_{n} \chi_{[u \leq 0]}+u_{-}$and $\partial_{t}\left(u-k_{n}\right)_{-} \chi_{[u \leq 0]}=$ $\partial_{t} u_{-}$. Taking into account $\left(u-k_{n}\right)_{-}\left(\cdot, t_{*}\right)=0$ in $K_{c_{2} R_{n}}$, we get

$$
\begin{aligned}
U( & \left.K_{c_{2} R_{n}}, t_{*}, 0,-\left(u-k_{n}\right)_{-} \zeta_{n}^{p}\right) \\
= & -\left.\int_{K_{c_{2} R_{n}}} v(\cdot, t) \chi_{[u \leq 0]}\left(u-k_{n}\right)_{-} \zeta_{n}^{p} \mathrm{~d} x\right|_{t=t_{*}} ^{0} \\
& +\int_{t_{*}}^{0} \int_{K_{c_{2} R_{n}}} v \chi_{[u \leq 0]} \frac{\partial\left(u-k_{n}\right)_{-}}{\partial t} \zeta_{n}^{p} \mathrm{~d} x \mathrm{~d} t \\
\leq & -\left.\int_{K_{c_{2} R_{n}}} v(\cdot, t) \chi_{[u \leq 0]} u_{-} \zeta_{n}^{p} \mathrm{~d} x\right|_{t=0} \\
& +\left.\bar{v} \int_{K_{c_{2} R_{n}}} u_{-} \zeta_{n}^{p} \mathrm{~d} x\right|_{t=t_{*}} ^{0} \leq 0,
\end{aligned}
$$

which proves the inequality (5.18). At this stage, we arrive at

$$
\begin{aligned}
\underset{t_{*}<t<0}{\operatorname{ess} \sup } & \int_{K_{c_{2} R_{n}} \times\{t\}}\left(u-k_{n}\right)_{-}^{2} \zeta_{n}^{p} \mathrm{~d} x+\iint_{Q_{n}}\left|D\left(u-k_{n}\right)_{-} \zeta_{n}\right|^{p} \mathrm{~d} x \mathrm{~d} t \\
& \leq \gamma_{1} \iint_{Q_{n}}\left(u-k_{n}\right)_{-}^{p}\left|D \zeta_{n}\right|^{p} \mathrm{~d} x \mathrm{~d} t .
\end{aligned}
$$

Set

$$
A_{n}=\iint_{Q_{n}} \chi_{\left[\left(u-k_{n}\right)_{->0}\right.} \mathrm{d} x \mathrm{~d} t \text { and } Y_{n}=\frac{A_{n}}{\left|Q_{n}\right|} .
$$

With this notation, the estimate (5.17) reads

$$
Y_{0} \leq v_{1} .
$$

Keeping in mind $\left(u-k_{n}\right)_{-} \leq 2^{-6} \omega$ and $\left|D \zeta_{n}\right| \leq 2^{n+4}\left(c_{2} R_{n}\right)^{-1}$, we deduce $\underset{t_{*}<t<0}{\operatorname{ess} \sup } \int_{K_{c_{2} R_{n}} \times\{t\}}\left(u-k_{n}\right)_{-}^{2} \zeta_{n}^{p} \mathrm{~d} x+\iint_{Q_{n}}\left|D\left(u-k_{n}\right)_{-} \zeta_{n}\right|^{p} \mathrm{~d} x \mathrm{~d} t \leq \gamma_{1} 2^{-2} \omega^{p} 2^{2 n} c_{2}^{-p} R_{n}^{-p} A_{n}$. Applying the parabolic Sobolev inequality (2.7), we obtain

$$
\begin{aligned}
& \iint_{Q_{n}}\left|\left(u-k_{n}\right)_{-} \zeta\right|^{p \frac{N+2}{N}} \mathrm{~d} x \mathrm{~d} t \\
& \quad \leq \gamma\left(\operatorname{ess}_{t_{*}<t<0} \int_{K_{C_{2} R_{n}} \times\{t\}}\left(u-k_{n}\right)_{-}^{2} \zeta_{n}^{2} \mathrm{~d} x\right)^{\frac{p}{N}} \\
& \iint_{Q_{n}}\left|D\left(u-k_{n}\right)_{-} \zeta_{n}\right|^{p} \mathrm{~d} x \mathrm{~d} t
\end{aligned}
$$




$$
\begin{aligned}
\leq & 8^{2\left(1+\frac{2}{N}\right)} \gamma \gamma_{1}^{1+\frac{2}{N}} 2^{n\left(2+\frac{4}{N}\right)} \omega^{p\left(1+\frac{p}{N}\right)} c_{2}^{-p\left(1+\frac{p}{N}\right)} \\
& R^{-p\left(1+\frac{p}{N}\right)} A_{n}^{1+\frac{p}{N}} .
\end{aligned}
$$

On the other hand, we estimate below

$$
\begin{gathered}
\iint_{Q_{n}}\left|\left(u-k_{n}\right)_{-} \zeta\right|^{p \frac{N+2}{N}} \mathrm{~d} x \mathrm{~d} t \geq \iint_{Q_{n+1}}\left|\left(u-k_{n}\right)_{-}\right|^{p \frac{N+2}{N}} \chi_{\left[\left(u-k_{n+1}\right)_{-}>0\right]} \mathrm{d} x \mathrm{~d} t \\
\geq\left(k_{n}-k_{n+1}\right)^{p \frac{N+2}{N}} A_{n+1} \geq\left(\frac{\omega}{2^{n+8}}\right)^{p \frac{N+2}{N}} A_{n+1} .
\end{gathered}
$$

Combining the estimates above we infer that

$$
Y_{n+1} \leq 2^{3 n\left(1+\frac{2}{N}\right)} 2^{22\left(1+\frac{2}{N}\right)} \gamma \gamma_{1}^{1+\frac{2}{N}} c_{2}^{-p\left(1+\frac{p}{N}\right)} \omega^{(p-2) \frac{p}{N}} R^{-p\left(1+\frac{p}{N}\right)} \frac{\left|Q_{n}\right|}{\left|Q_{n+1}\right|}\left|Q_{n}\right|^{\frac{p}{N}} Y_{n}^{1+\frac{p}{N}} .
$$

Taking into account $\left|Q_{n}\right|=L_{2}^{N} d_{*}^{N} R_{n}^{N} t_{*}, 8^{-1} R \leq R_{n} \leq 4^{-1} R, t_{*} \leq L_{1} d R^{p}$ and the relation (4.2), we obtain

$$
\begin{aligned}
Y_{n+1} & \leq 2^{3 n\left(1+\frac{2}{N}\right)} 2^{22\left(1+\frac{2}{N}\right)+N} \gamma \gamma_{1}^{1+\frac{2}{N}} \omega^{(p-2) \frac{p}{N}}\left(\frac{L_{1}}{L_{2}^{p}}\right)^{\frac{p}{N}}\left(\frac{d}{d_{*}^{p}}\right)^{\frac{p}{N}} Y_{n}^{1+\frac{p}{N}} \\
& \leq 2^{3 n\left(1+\frac{2}{N}\right)} 2^{22\left(1+\frac{2}{N}\right)+N} \gamma \gamma_{1}^{1+\frac{2}{N}} Y_{n}^{1+\frac{p}{N}},
\end{aligned}
$$

since

$$
\left|Q_{n}\right| \leq L_{1} L_{2}^{N} d_{*}^{N} d R^{N+p}, \quad \frac{\left|Q_{n}\right|}{\left|Q_{n+1}\right|} \leq 2^{N} \quad \text { and } \quad \frac{L_{1}}{L_{2}^{p}} \leq \frac{\nu_{1}}{2^{N+12} \gamma_{2}}<1 .
$$

Recalling from (5.16) and (5.19) that

$$
Y_{0} \leq v_{1}<\left(2^{3\left(1+\frac{2}{N}\right)}\right)^{-\frac{N^{2}}{p^{2}}}\left(2^{22\left(1+\frac{2}{N}\right)+N} \gamma \gamma_{1}^{1+\frac{2}{N}}\right)^{-\frac{N}{p}} .
$$

By the lemma on fast geometric convergence of sequences (cf. [5, Chapter I, Lemma 4.1]), we conclude that $Y_{n} \rightarrow 0$ as $n \rightarrow \infty$, which proves the proposition.

Henceforth, we choose $L_{1}=2$. For such a choice of $L_{1}$, we determine $L_{2}$ by

$$
L_{2}=8\left(2^{N+12} \gamma_{2}\right)^{\frac{1}{p}} v_{1}^{-\frac{1}{p}}
$$

where $v_{1}$ is the constant chosen according to (5.16). With these choices, and taking (5.15) into account, we obtain the desired estimate (4.3) under the assumption of the first alternative.

\section{The second alternative}

In this section, we will establish the estimate (4.3) for the second alternative. We begin with the following lemma, the proof of which we omit.

Lemma 6.1 Let $v_{0}$ be a constant chosen according to (5.4), and assume that (4.5) holds for some fixed $\bar{t} \in I_{\omega}$ and $\bar{x} \in K_{\omega}$. There exists a time level $t_{0} \in\left[\bar{t}-d R^{p}, \bar{t}-\frac{1}{2} v_{0} d R^{p}\right]$ such that

$$
\left|\left\{x \in \bar{x}+K_{d_{*} R}: u>\mu_{+}-\frac{\omega}{4}\right\}\right| \leq\left(\frac{1-v_{0}}{1-\frac{1}{2} v_{0}}\right)\left|K_{d_{*} R}\right| .
$$


The estimate (6.1) is the starting point for the analysis of the second alternative, and we can now ignore inequality (4.5). Next, since $\mu_{+} \geq\left|\mu_{-}\right|$and $\mu_{+}-\mu_{-}>\frac{\omega}{2}$, then $\mu_{+} \geq 0$ and $4 \mu_{+}>\omega$. For $k \geq \mu_{+}-\frac{\omega}{4}$, we observe that if $(u-k)_{+}>0$, then $u>k>0$. Therefore, we can always discard the term involving $U$ in the energy estimates. Let $m_{2} \geq 2$ be the quantity that will be determined in Sect. 6.2. We provide the following lemma regarding the expansion of positivity in the time direction.

Lemma 6.2 There exists $\tilde{n}>1$ depending only upon the data and $v_{0}$, and independent of $m_{2}$ such that

$$
\left|\left\{x \in \bar{x}+K_{d_{*} R}: u>\mu_{+}-\frac{\omega}{2^{m_{2}+\tilde{n}}}\right\}\right| \leq\left(1-\left(\frac{\nu_{0}}{2}\right)^{2}\right)\left|K_{d_{*} R}\right|
$$

for all $t \in\left(t_{0}, \tilde{t}\right)$, where

$$
\tilde{t}=t_{0}+\frac{\nu_{0}}{2} 2^{-\tilde{n p}(2-p)}\left(\frac{\omega}{2^{m_{2}}}\right)^{(1-p)(2-p)} R^{p} .
$$

Proof Without loss of generality, we assume that $\bar{x}=0$. From (6.3), we observe that

$$
K_{d_{*} R} \times\left(t_{0}, \tilde{t}\right] \subset(0, \bar{t})+Q\left(d R^{p}, d_{*} R\right) .
$$

This enables us to use the logarithmic estimate (3.4) over the cylinder $K_{d_{*} R} \times\left(t_{0}, \tilde{t}\right]$. Set $k=\mu_{+}-\frac{\omega}{2^{m_{2}}}$ and $c=\frac{\omega}{2^{m_{2}+\tilde{n}}}$ where $\tilde{n}>1$ is to be determined. We consider the logarithmic function

$$
\psi^{+}=\ln ^{+}\left(\frac{\frac{\omega}{2^{m}}}{\frac{\omega}{2^{m}{ }^{2}}-(u-k)_{+}+c}\right) .
$$

Then, we have $\psi^{+} \leq \tilde{n} \ln 2$ and

$$
\begin{aligned}
\left|\left(\psi^{+}\right)^{\prime}\right|^{2-p} & \leq\left(\frac{\omega}{2^{m_{2}-1}}\right)^{(p-1)(2-p)}\left(\frac{2^{m_{2}+\tilde{n}}}{\omega}\right)^{p(2-p)} \\
& =2^{(p-1)(2-p)}\left(\frac{\omega}{2^{m_{2}}}\right)^{(p-1)(2-p)} d_{*}^{p} 2^{\tilde{n} p(2-p)} .
\end{aligned}
$$

Choose a piecewise smooth cutoff function $\zeta(x)$, defined in $K_{d_{*} R}$, and satisfying $0 \leq \zeta \leq 1$ in $K_{d_{*} R}, \zeta \equiv 1$ in $K_{(1-\sigma) d_{*} R}$ and $|D \zeta| \leq\left(\sigma d_{*} R\right)^{-1}$, where $\sigma \in(0,1)$ is to be determined. Since $k>\mu_{+}-\frac{\omega}{4}$, then $U\left(K_{d_{*} R}, t_{0}, \tilde{t}, 2 \psi^{+}\left(\psi^{+}\right)^{\prime} \zeta^{p}\right)=0$. Then, we deduce from (3.4) the logarithmic estimate

$$
\begin{gathered}
\underset{t_{0}<t<\tilde{t}}{\operatorname{ess} \sup _{K_{d_{*} R} \times\{t\}}} \int\left(\psi^{+}\right)^{2} \zeta^{p} \mathrm{~d} x \leq \int_{K_{d_{*} R} \times\left\{t_{0}\right\}}\left(\psi^{+}\right)^{2} \zeta^{p} \mathrm{~d} x \\
+\gamma_{2} \int_{t_{0}}^{\tilde{t}} \int_{K_{d_{*} R}} \psi^{+}\left|\left(\psi^{+}\right)^{\prime}\right|^{2-p}|D \zeta|^{p} \mathrm{~d} x \mathrm{~d} t .
\end{gathered}
$$

Recalling the definition of $\tilde{t}$ and (6.1), we obtain

$$
\begin{aligned}
\underset{t_{0}<t<\tilde{t}}{\operatorname{ess} \sup } & \int_{K_{d_{*} R} \times\{t\}}\left(\psi^{+}\right)^{2} \zeta^{p} \mathrm{~d} x \\
& \leq \tilde{n}^{2}(\ln 2)^{2}\left|\left\{x \in K_{d_{*} R}: u>k\right\}\right|
\end{aligned}
$$




$$
\begin{aligned}
& +\gamma_{2} \frac{2 \tilde{n} \ln 2}{\sigma^{p} d_{*}^{p} R^{p}}\left(\frac{\omega}{2^{m_{2}}}\right)^{(p-1)(2-p)} d_{*}^{p} 2^{\tilde{n p}(2-p)}\left(\tilde{t}-t_{0}\right)\left|K_{d_{*} R}\right| \\
\leq & \left(\tilde{n}^{2}(\ln 2)^{2}\left(\frac{1-v_{0}}{1-\frac{1}{2} v_{0}}\right)+v_{0}(\ln 2) \gamma_{2} \frac{\tilde{n}}{\sigma^{p}}\right)\left|K_{d_{*} R}\right|
\end{aligned}
$$

At this stage, we proceed similarly as in [6, page 938-939]. To estimate below the integral on the left-hand side, we consider a smaller set defined by

$$
S=\left\{x \in K_{(1-\sigma) d_{*} R}: u>\mu_{+}-\frac{\omega}{2^{m_{2}+\tilde{n}}}\right\} \subset K_{(1-\sigma) d_{*} R} .
$$

On such a set, $\zeta \equiv 1$ and

$$
\psi^{+}=\ln ^{+}\left(\frac{\frac{\omega}{2^{m}}}{\frac{\omega}{2^{m}}-(u-k)_{+}+\frac{\omega}{2^{m_{2}+\tilde{n}}}}\right) \geq(\tilde{n}-1) \ln 2 .
$$

It follows that

$$
\begin{aligned}
& \left|\left\{x \in \bar{x}+K_{d_{*} R}: u>\mu_{+}-\frac{\omega}{2^{m_{2}+\tilde{n}}}\right\}\right| \\
& \leq\left(\left(\frac{\tilde{n}}{\tilde{n}-1}\right)^{2}\left(\frac{1-v_{0}}{1-\frac{1}{2} v_{0}}\right)+\frac{8 \gamma_{2}}{\tilde{n} \sigma^{p}}+N \sigma\right)\left|K_{d_{*} R}\right|
\end{aligned}
$$

for all $t \in\left(t_{0}, \tilde{t}\right)$. To prove the lemma, choose

$$
\sigma=\frac{3}{8 N} v_{0}^{2} \text { and } \tilde{n}=\frac{8 \gamma_{2}}{v_{0}^{6}} .
$$

With this choice, we see that $\tilde{n}$ is independent of $m_{2}$.

\subsection{Expansion of positivity in the space variable}

In this subsection, we will establish an expansion of positivity result for the weak solutions in a larger cylinder. We have to work with an equation in dimensionless form, and our proof is in the spirit of $[1,3,5,6]$. To start with, we introduce a change of variables

$$
x^{\prime}=\frac{x-\bar{x}}{2 c_{2} R} \text { and } t^{\prime}=4^{p}\left(\frac{t-\tilde{t}}{\tilde{t}-t_{0}}\right) .
$$

This transformation maps $\left[\bar{x}+K_{d_{*} R}\right] \times\left(t_{0}, \tilde{t}\right) \rightarrow K_{\frac{1}{2 L_{2}}} \times\left(-4^{p}, 0\right)$ and $\left[\bar{x}+K_{8 c_{2} R}\right] \times\left(t_{0}, \tilde{t}\right) \rightarrow$ $K_{4} \times\left(-4^{p}, 0\right)$. Next, we set the new functions

$$
\tilde{u}\left(x^{\prime}, t^{\prime}\right)=u(x, t) \text { and } \bar{u}\left(x^{\prime}, t^{\prime}\right)=\left(\tilde{u}\left(x^{\prime}, t^{\prime}\right)-\mu_{+}\right)\left(\frac{2^{m_{2}+\tilde{n}-1}}{\omega}\right) .
$$

With these notations, the estimate (6.2) can be rewritten as

$$
\left|\left\{x^{\prime} \in K_{\frac{1}{2 L_{2}}}: \bar{u}\left(x^{\prime}, t^{\prime}\right)<-\frac{1}{2}\right\}\right| \geq\left(\frac{v_{0}}{2}\right)^{2}\left|K_{\frac{1}{2 L_{2}}}\right|
$$


for all $t^{\prime} \in\left(-4^{p}, 0\right]$. On the other hand, we set $\tilde{w}\left(x^{\prime}, t^{\prime}\right)=w(x, t)$. The function $v(x, t)$ in (3.1) can be written in the new variables as

$$
\tilde{v}\left(x^{\prime}, t^{\prime}\right)= \begin{cases}\bar{v}, & \text { on }\left\{\bar{u}<-\mu_{+} \frac{2^{m_{2}+\tilde{n}-1}}{\omega}\right\}, \\ -\tilde{w}\left(x^{\prime}, t^{\prime}\right), & \text { on }\left\{\bar{u}=-\mu_{+} \frac{2^{m_{2}+\tilde{n}-1}}{\omega}\right\} .\end{cases}
$$

For any $\varphi \in W_{p}\left(Q_{4}\right)$ and $\left[t_{1}, t_{2}\right] \subset\left(-4^{p}, 0\right]$, we rewrite the weak form (3.1) in terms of the new variables and new functions as follows:

$$
\begin{aligned}
-\frac{2^{m_{2}+\tilde{n}-1}}{\omega} \int_{K_{4}} \tilde{v}\left(\cdot, t^{\prime}\right) \chi\left\{\bar{u} \leq-\mu_{+} \frac{2^{m_{2}+\tilde{n}-1}}{\omega}\right\} & \left.\varphi\left(\cdot, t^{\prime}\right) \mathrm{d} x^{\prime}\right|_{t^{\prime}=t_{1}} ^{t_{2}} \\
& +\frac{2^{m_{2}+\tilde{n}-1}}{\omega} \int_{t_{1}}^{t_{2}} \int_{K_{4}} \tilde{v} \chi\left\{\bar{u} \leq-\mu_{+} \frac{2^{m_{2}+\tilde{n}-1}}{\omega}\right\} \frac{\partial \varphi}{\partial t^{\prime}} \mathrm{d} x^{\prime} \mathrm{d} t^{\prime} \\
& +\int_{t_{1}}^{t_{2}} \int_{K_{4}}\left(\varphi \frac{\partial \bar{u}}{\partial t^{\prime}}+\bar{A}\left(x^{\prime}, t^{\prime}, \bar{u}, D \bar{u}\right) \cdot D_{x^{\prime}} \varphi\right) \mathrm{d} x^{\prime} \mathrm{d} t^{\prime}=0,
\end{aligned}
$$

where

$$
\begin{aligned}
& \bar{A}\left(x^{\prime}, t^{\prime}, \bar{u}, D \bar{u}\right)=\frac{2^{m_{2}+\tilde{n}}\left(\tilde{t}-t_{0}\right)}{4^{p+1} c_{2} \omega R} \\
& A\left(\bar{x}+2 c_{2} R x^{\prime}, \tilde{t}+\frac{\tilde{t}-t_{0}}{4^{p}} t^{\prime}, \frac{\omega}{2^{m_{2}+\tilde{n}-1}} \bar{u}+\mu_{+}, \frac{\omega}{2^{m_{2}+\tilde{n}_{2}} c_{2}} D_{x^{\prime}} \bar{u}\right) .
\end{aligned}
$$

From (1.3), we obtain the structure conditions for $\bar{A}$ as follows:

$$
\left\{\begin{array}{l}
\bar{A}\left(x^{\prime}, t^{\prime}, \bar{u}, D \bar{u}\right) \cdot D \bar{u} \geq C_{0} \Lambda(\omega)|D \bar{u}|^{p}, \\
\left|\bar{A}\left(x^{\prime}, t^{\prime}, \bar{u}, D \bar{u}\right)\right| \leq C_{1} \Lambda(\omega)|D \bar{u}|^{p-1},
\end{array}\right.
$$

where

$$
\Lambda(\omega)=\frac{2^{\left(m_{2}+\tilde{n}\right)(2-p)} \omega^{p-2}\left(\tilde{t}-t_{0}\right)}{4^{p+1} c_{2}^{p} R^{p}} .
$$

According to (6.3), the above identity reads

$$
\Lambda(\omega)=4^{-p-\frac{3}{2}} \nu_{0} L_{2}^{-p} 2^{\tilde{n}(1-p)(2-p)},
$$

which is independent of $m_{2}$. By abuse of the notation, we write $(x, t)$ instead of the new variables. The next lemma is an analogue of [5, Chapter II, Lemma 1.1].

Lemma 6.3 Let $k \in(-1,0)$. Then, there holds

$$
\int_{t_{1}}^{t_{2}} \int_{K_{4}} \varphi \frac{\partial(\bar{u}-k)_{+}}{\partial t}+\bar{A}\left(x, t, k+(\bar{u}-k)_{+}, D(\bar{u}-k)_{+}\right) \cdot D \varphi d x d t \leq 0
$$

for all $\varphi \in W_{p}\left(Q_{4}\right)$ and $\varphi \geq 0$.

Proof We proceed similarly as in [5, page 18-19]. In (6.7), choose the testing function

$$
\varphi_{\varepsilon}=\varphi \frac{(\bar{u}-k)_{+}}{(\bar{u}-k)_{+}+\varepsilon}, \quad \varepsilon>0 .
$$


In view of $\omega<4 \mu_{+}$, we find that the first two terms in (6.7) vanish and there holds

$$
\begin{aligned}
\int_{t_{1}}^{t_{2}} \int_{K_{4}} \varphi_{\varepsilon} & \frac{\partial \bar{u}}{\partial t}+\frac{(\bar{u}-k)_{+}}{(\bar{u}-k)_{+}+\varepsilon} \bar{A}(x, t, \bar{u}, D \bar{u}) \cdot D \varphi \mathrm{d} x \mathrm{~d} t \\
\leq & -C_{0} \Lambda(\omega) \int_{t_{1}}^{t_{2}} \int_{K_{4}} \frac{\varepsilon \varphi}{\left((\bar{u}-k)_{+}+\varepsilon\right)^{2}}\left|D(\bar{u}-k)_{+}\right|^{p} \mathrm{~d} x \mathrm{~d} t \\
\leq & 0 .
\end{aligned}
$$

Consequently, the inequality (6.10) follows by passing to the limit $\varepsilon \downarrow 0$.

With the previous result in hand, we can now present the following lemma which concerns the expansion of positivity in the space variable. Since the constants in the proof should be uniformly bounded in $p$ when $p \rightarrow 2$, we employ the idea from Alkhutov and Zhikov [1]. Moreover, this approach enables us to obtain explicit estimates for the parameters that will play a crucial role in the next subsection.

Lemma 6.4 Suppose that $\frac{3}{2} \leq p<2$. For any $v \in(0,1)$, there exists $\tilde{m}>1$ depending only upon the data, $v$ and $v_{0}$, such that

$$
\left|\left\{x \in K_{2}: \bar{u}(x, t) \geq-2^{-\tilde{m}}\right\}\right| \leq v\left|K_{2}\right|
$$

for all $t \in\left[-2^{p}, 0\right]$.

Proof Before proceeding to the proof, we introduce the auxiliary functions

$$
\phi_{k}(\bar{u})=\int_{0}^{(\bar{u}-k)_{+}} \frac{\mathrm{d} \tau}{(-(1-\delta) k-\tau+V)^{p-1}}
$$

and

$$
\psi_{k}(\bar{u})=\ln \left(\frac{-(1-\delta) k+V}{-k(1-\delta)-(\bar{u}-k)_{+}+V}\right)
$$

where $V=(-k)(-\delta)^{\frac{p}{p-1}}$ and $k, \delta \in\left(-\frac{1}{8}, 0\right)$ are to be determined. Recalling the definition of $\bar{u}$, we have $\bar{u} \leq 0$ and $(\bar{u}-k)_{+} \leq-k$. Moreover, we obtain the inequality

$$
(-k(1-\delta)-\tau+V)^{p-1} \leq(-k(1-\delta)-\tau)^{p-1}+V^{p-1} \leq(1-\delta)(-k(1-\delta)-\tau)^{p-1}
$$

for any $0 \leq \tau \leq-k$. Taking into account

$$
\phi_{k}(\bar{u})=\frac{1}{2-p}(-k(1-\delta)+V)^{2-p}\left\{1-\left(1-\frac{(\bar{u}-k)_{+}}{-k(1-\delta)+V}\right)^{2-p}\right\}
$$

and $1-(1-z)^{2-p} \leq-(2-p) \ln (1-z)$, we conclude that

$$
\phi_{k}(\bar{u}) \leq \ln \frac{-k(1-\delta)+V}{-k(1-\delta)-(\bar{u}-k)_{+}+V} \leq \ln \frac{-k(1-\delta)+V}{-k(-\delta)+V} \leq \ln \frac{1-\delta}{-\delta} .
$$

Let $\zeta=\zeta_{1}(x) \zeta_{2}(t)$ be a piecewise smooth cutoff function defined in $Q_{4}$. Suppose that $0 \leq \zeta \leq 1$ in $Q_{4}, \zeta \equiv 1$ in $Q_{2}, \zeta \equiv 0$ on $\partial_{P} Q_{4},\left|D \zeta_{1}\right| \leq 1,0 \leq \partial_{t} \zeta_{2} \leq 1$ and the sets $\left\{x \in K_{4}: \zeta_{1}(x)>-k\right\}$ are convex for all $k \in\left(-\frac{1}{8}, 0\right)$. Testing the weak formulation (6.10) with the function

$$
\varphi(x, t)=\frac{\zeta_{1}(x)^{p} \zeta_{2}(t)^{p}}{\left(-(1-\delta) k-(\bar{u}(x, t)-k)_{+}+V\right)^{p-1}}
$$


and taking (6.8) into account, we have

$$
\begin{array}{r}
\int_{t_{1}}^{t_{2}} \int_{K_{4}} \zeta^{p} \frac{\partial \phi_{k}(\bar{u})}{\partial t}+C_{0} \Lambda(\omega) \frac{(p-1) \zeta^{p}\left|D(\bar{u}-k)_{+}\right|^{p}}{\left(-k(1-\delta)-(\bar{u}-k)_{+}+V\right)^{p}} \mathrm{~d} x \mathrm{~d} t \\
\leq C_{1} \Lambda(\omega) \int_{t_{1}}^{t_{2}} \int_{K_{4}} \frac{p \zeta^{p-1}|D \zeta|\left|D(\bar{u}-k)_{+}\right|^{p-1}}{\left(-k(1-\delta)-(\bar{u}-k)_{+}+V\right)^{p-1}} \mathrm{~d} x \mathrm{~d} t
\end{array}
$$

for any $\left[t_{1}, t_{2}\right] \subset\left(-4^{p}, 0\right]$. In view of $p \geq \frac{3}{2}, \Lambda(\omega) \leq 1$, and therefore, Young's inequality and (6.12) allow us to conclude that

$$
\begin{aligned}
& \left.\int_{K_{4}} \zeta^{p} \phi_{k}(\bar{u}) \mathrm{d} x\right|_{t=t_{1}} ^{t_{2}}+\frac{C_{0} \Lambda(\omega)}{4} \int_{t_{1}}^{t_{2}} \int_{K_{4}} \zeta^{p}\left|D \psi_{k}(\bar{u})\right|^{p} \mathrm{~d} x \mathrm{~d} t \\
& \quad \leq \kappa_{p} \Lambda(\omega) \int_{t_{1}}^{t_{2}} \int_{K_{4}} \zeta^{p}|D \zeta|^{p} \mathrm{~d} x \mathrm{~d} t+\int_{t_{1}}^{t_{2}} \int_{K_{4}} p \zeta^{p-1} \phi_{k}(\bar{u}) \frac{\partial \zeta}{\partial t} \mathrm{~d} x \mathrm{~d} t \\
& \quad \leq C_{2}\left(t_{2}-t_{1}\right) \ln \frac{1-\delta}{-\delta}
\end{aligned}
$$

where

$$
\kappa_{p}=p C_{1}\left(\frac{2 p C_{1}}{(p-1) C_{0}}\right)^{p-1} \text { and } C_{2}=\frac{16 C_{1}^{2}}{C_{0}} .
$$

Next, we observe that

$$
\left\{x \in K_{4}: \bar{u}(x, t)<-\frac{1}{2}\right\} \cap K_{\frac{1}{2 L_{2}}} \subset\left\{x \in K_{4}: \phi_{k}(\bar{u})=0\right\} \cap\left\{x \in K_{4}: \zeta_{1}(x)=1\right\}
$$

for any $t \in\left(-4^{p}, 0\right]$. Applying Lemma 2.3 to each of the time slices and keeping in mind (6.5), we deduce

$$
\int_{K_{4}} \zeta_{1}^{p}\left|\psi_{k}(\bar{u})\right|^{p} \mathrm{~d} x \leq 4^{3 N} \gamma^{2} L_{2}^{2(N-1)} v_{0}^{-2 \frac{N-1}{N}} \int_{K_{4}} \zeta_{1}^{p}\left|D \psi_{k}(\bar{u})\right|^{p} \mathrm{~d} x .
$$

Multiplying both sides of the above inequality by $\zeta_{2}(t)^{p}$ and integrating over $\left[t_{1}, t_{2}\right]$, we obtain

$$
\left.\int_{K_{4}} \zeta^{p} \phi_{k}(\bar{u}) \mathrm{d} x\right|_{t=t_{1}} ^{t_{2}}+\bar{\Lambda}(\omega) \int_{t_{1}}^{t_{2}} \int_{K_{4}} \zeta^{p} \psi_{k}(\bar{u})^{p} \mathrm{~d} x \mathrm{~d} t \leq C_{2}\left(t_{2}-t_{1}\right) \ln \frac{1-\delta}{-\delta}
$$

where

$$
\bar{\Lambda}(\omega)=\gamma_{3} v_{0}^{2 \frac{N-1}{N}} \Lambda(\omega) \text { and } \gamma_{3}=4^{-3 N-1} \gamma^{-2} L_{2}^{-2(N-1)} C_{0}^{-1} .
$$

Recalling that $u \in C_{\mathrm{loc}}\left(0, T ; L_{\mathrm{loc}}^{2}(\Omega)\right)$, we obtain $\phi_{k}(\bar{u}), \psi_{k}(\bar{u})^{p} \in C_{\mathrm{loc}}\left(0, T ; L_{\mathrm{loc}}^{1}(\Omega)\right)$ and for any $t \in\left(-4^{p}, 0\right]$ there holds

$$
\frac{\mathrm{d}^{-}}{\mathrm{d} t} \int_{K_{4} \times\{t\}} \zeta^{p} \phi_{k}(\bar{u}) \mathrm{d} x+\bar{\Lambda}(\omega) \int_{K_{4} \times\{t\}} \zeta^{p} \psi_{k}(\bar{u})^{p} \mathrm{~d} x \leq C_{2} \ln \frac{1-\delta}{-\delta},
$$

where

$$
\frac{\mathrm{d}^{-}}{\mathrm{d} t} \int_{K_{4} \times\{t\}} \zeta^{p} \phi_{k}(\bar{u}) \mathrm{d} x=\lim \sup _{h \rightarrow 0+} \frac{1}{h}\left(\int_{K_{4} \times\{t\}} \zeta^{p} \phi_{k}(\bar{u}) \mathrm{d} x-\int_{K_{4} \times\{t-h\}} \zeta^{p} \phi_{k}(\bar{u}) \mathrm{d} x\right) .
$$

At this stage, we introduce the quantities

$$
Y_{i}=\sup _{-4^{p} \leq t \leq 0} \int_{K_{4} \cap\left[\bar{u}>-|\delta|^{i}\right]} \zeta^{p}(x, t) \mathrm{d} x, \quad i=1,2, \ldots
$$


To prove this lemma, it suffices to determine constants $\delta$ and $i_{*}$ depending only upon the data, $v$ and $v_{0}$, such that $Y_{i_{*}} \leq v$.

Fix $i \in \mathbb{N}$, we choose $k=-|\delta|^{i}$ and $V=|\delta|^{i+\frac{p}{p-1}}$. For any $\varepsilon>0$, there exists $t_{0} \in\left(-4^{p}, 0\right]$ such that

$$
\int_{K_{4} \cap\left[\bar{u}>-|\delta|^{i+1}\right]} \zeta^{p}\left(\cdot, t_{0}\right) \mathrm{d} x \geq Y_{i+1}-\varepsilon .
$$

Let

$$
\mathcal{C}^{+}=\left\{t \in\left(-4^{p}, 0\right]: \frac{\mathrm{d}^{-}}{\mathrm{d} t} \int_{K_{4} \times\{t\}} \zeta^{p} \phi_{k}(\bar{u}) \mathrm{d} x \geq 0\right\} .
$$

In the case $t_{0} \in \mathcal{C}^{+}$, we choose $\varepsilon \in(0, v / 2]$. In (6.15), take $t=t_{0}, k=-|\delta|^{i}$, and there holds

$$
\bar{\Lambda}(\omega) \int_{K_{4}} \zeta^{p}\left(\cdot, t_{0}\right) \psi_{-|\delta|^{i}}(\bar{u})^{p}\left(\cdot, t_{0}\right) \mathrm{d} x \leq C_{2} \ln \frac{1-\delta}{-\delta} .
$$

To estimate below the integral on the left-hand side, we consider the domain of integration $K_{4} \cap\left[\bar{u}>-|\delta|^{i+1}\right]$. On such a set, $\left(\bar{u}+|\delta|^{i}\right)_{+}>|\delta|^{i}-|\delta|^{i+1}$. Taking into account $V \leq|\delta|^{i+1}$, we deduce

$$
\psi_{k}(\bar{u})=\ln \left(\frac{(1-\delta)|\delta|^{i}+V}{(1-\delta)|\delta|^{i}-\left(\bar{u}+|\delta|^{i}\right)_{+}+V}\right) \geq \ln \frac{1+|\delta|}{3|\delta|} .
$$

Since $|\delta|<\frac{1}{8}$, then there holds

$$
\frac{1+|\delta|}{|\delta|} \leq\left(\frac{1+|\delta|}{3|\delta|}\right)^{2}
$$

We use these estimates to conclude that

$$
\bar{\Lambda}(\omega) \int_{K_{4} \cap\left[\bar{u}>-|\delta|^{i+1}\right]} \zeta^{p}\left(\cdot, t_{0}\right) \mathrm{d} x \leq 2 C_{2}\left(\ln \frac{1+|\delta|}{3|\delta|}\right)^{1-p}
$$

and therefore,

$$
Y_{i+1} \leq \frac{v}{2}+\frac{2 C_{2}}{\bar{\Lambda}(\omega)}\left(\ln \frac{1+|\delta|}{3|\delta|}\right)^{1-p} .
$$

Then, $Y_{i+1} \leq v$, provided

$$
|\delta| \leq \frac{1}{3}\left(\exp \left(\frac{\nu \bar{\Lambda}(\omega)}{2 C_{2}}\right)^{\frac{1}{1-p}}-\frac{1}{3}\right)^{-1} .
$$

To this end, we choose

$$
|\delta|=\frac{1}{30} \exp \left\{-64\left(\frac{4 C_{2}}{v \bar{\Lambda}(\omega)}\right)^{2}\right\} .
$$

We now turn our attention to the case $t_{0} \notin \mathcal{C}^{+}$. Denote by $t_{*}$ the least upper bound of the set

$$
\mathcal{C}_{t_{0}}^{+}=\left\{t \in \mathcal{C}^{+}: t<t_{0}\right\}
$$


In the case $t_{0}=t_{*}$, there exists a sequence $t_{k}^{\prime} \in \mathcal{C}_{t_{0}}^{+}$such that $t_{k}^{\prime} \rightarrow t_{0}$ as $k \rightarrow \infty$, and

$$
\bar{\Lambda}(\omega) \int_{K_{4}} \zeta^{p}\left(\cdot, t_{k}^{\prime}\right) \psi_{-|\delta|^{i}}(\bar{u})^{p}\left(\cdot, t_{k}^{\prime}\right) \mathrm{d} x \leq C_{2} \ln \frac{1-\delta}{-\delta} .
$$

Passing to the limit $t_{k}^{\prime} \rightarrow t_{0}$ and taking into account that $\psi_{-|\delta|^{i}}(\bar{u})^{p} \in C_{\mathrm{loc}}\left(0, T ; L_{\mathrm{loc}}^{1}(\Omega)\right)$, we deduce

$$
\bar{\Lambda}(\omega) \int_{K_{4}} \zeta^{p}\left(\cdot, t_{0}\right) \psi_{-|\delta|^{i}}(\bar{u})^{p}\left(\cdot, t_{0}\right) \mathrm{d} x \leq C_{2} \ln \frac{1-\delta}{-\delta} .
$$

We may now repeat the same arguments as in the previous proof and obtain $Y_{i+1} \leq v$, provided that $\delta$ satisfies (6.16).

Finally, we come to the case $t_{0}>t_{*}$. Let $j$ be an integer that can be determined a priori only in terms of the data, $v$ and $v_{0}$. Let us initially assume $Y_{i}>v$ for all $i=1,2, \ldots, j$. Next, we claim that

$$
Y_{i+1} \leq(1-|\delta|) Y_{i} \quad i=1,2, \ldots, j .
$$

Fix $i \in\{1,2, \ldots, j\}$. Since $t_{0}>t_{*}$, there holds

$$
\frac{\mathrm{d}^{-}}{\mathrm{d} t} \int_{K_{4}} \zeta^{p}(\cdot, t) \phi_{-|\delta|^{i}}(\bar{u})(\cdot, t) \mathrm{d} x \leq 0
$$

for any $t \in\left(t_{*}, t_{0}\right)$. It follows that

$$
\int_{K_{4}} \zeta^{p}\left(\cdot, t_{0}\right) \phi_{-|\delta|^{i}}(\bar{u})\left(\cdot, t_{0}\right) \mathrm{d} x \leq \int_{K_{4}} \zeta^{p}\left(\cdot, t_{*}\right) \phi_{-|\delta|^{i}}(\bar{u})\left(\cdot, t_{*}\right) \mathrm{d} x .
$$

Recalling the definition of $t_{*}$, we have

$$
\bar{\Lambda}(\omega) \int_{K_{4}} \zeta^{p}\left(\cdot, t_{*}\right) \psi_{-|\delta|^{i}}(\bar{u})^{p}\left(\cdot, t_{*}\right) \mathrm{d} x \leq C_{2} \ln \frac{1-\delta}{-\delta}=: C_{\delta},
$$

with the obvious meaning of $C_{\delta}$. Consider the set $K_{4} \cap\left[\left(\bar{u}+|\delta|^{i}\right)_{+}>\tau|\delta|^{i}\right]$, where $\tau \in[0,1]$. On this set

$$
\psi_{-|\delta|^{i}}(\bar{u})=\ln \left(\frac{(1-\delta)|\delta|^{i}+V}{(1-\delta)|\delta|^{i}-\left(\bar{u}+|\delta|^{i}\right)_{+}+V}\right) \geq \ln \frac{1+2|\delta|}{1+2|\delta|-\tau},
$$

since $V \leq|\delta|^{i+1}$. Combining this estimate with (6.19), we obtain

$$
\int_{K_{4} \cap\left[\left(\bar{u}+|\delta|^{i}\right)_{+}>\tau|\delta|^{i}\right]} \zeta^{p}\left(\cdot, t_{*}\right) \mathrm{d} x \leq \frac{C_{\delta}}{\bar{\Lambda}(\omega)}\left(\ln \frac{1+2|\delta|}{1+2|\delta|-\tau}\right)^{-p} .
$$

At this stage, we set

$$
\tau_{*}=\frac{\exp \left(\frac{C_{\delta}}{\bar{\Lambda}(\omega) Y_{i}}\right)^{\frac{1}{p}}-1}{\exp \left(\frac{C_{\delta}}{\bar{\Lambda}(\omega) Y_{i}}\right)^{\frac{1}{p}}}(1+2|\delta|) .
$$

Since $Y_{i}>v$, we have

$$
\tau_{*}<\frac{\exp \left(\frac{C_{\delta}}{\bar{\Lambda}(\omega) v}\right)^{\frac{1}{p}}-1}{\exp \left(\frac{C_{\delta}}{\bar{\Lambda}(\omega) v}\right)^{\frac{1}{p}}}(1+2|\delta|)=: \sigma(1+2|\delta|),
$$


with the obvious meaning of $\sigma$. For a technical reason, we introduce a constant $\sigma^{\prime}>\sigma$ defined by

$$
\sigma^{\prime}=\frac{\exp \left(\frac{2 C_{\delta}}{\bar{\Lambda}(\omega) v}\right)^{\frac{1}{p}}-1}{\exp \left(\frac{2 C_{\delta}}{\bar{\Lambda}(\omega) v}\right)^{\frac{1}{p}}}
$$

At this point, we claim that

$$
\sigma^{\prime}(1+2|\delta|)<1-\sqrt{|\delta|} .
$$

For the value of $|\delta|$ given by (6.16), we have

$$
|\delta|<\frac{1}{2} \exp \left\{-2^{\frac{p}{p-1}}\left(\frac{4 C_{2}}{v \bar{\Lambda}(\omega)}\right)^{\frac{1}{p-1}}\right\}
$$

for all $\frac{3}{2} \leq p<2$. Then, there holds

$$
\left(\ln \left(\frac{1}{2|\delta|}\right)^{\frac{4 C_{2}}{v \bar{\Lambda}(\omega)}}\right)^{\frac{1}{p}} \leq \ln \left(\frac{1}{2|\delta|}\right)^{\frac{1}{2}} .
$$

From (6.16), we observe that $|\delta|<(2+2 \sqrt{2})^{-1}$ and this implies that $|\delta|^{-1}(1+|\delta|)<$ $(2|\delta|)^{-2}$. Combining this inequality with $(6.21)$, we obtain

$$
\exp \left(\frac{2 C_{\delta}}{\bar{\Lambda}(\omega) \nu}\right)^{\frac{1}{p}}=\exp \left(\frac{2 C_{2}}{\bar{\Lambda}(\omega) \nu} \ln \frac{1+|\delta|}{|\delta|}\right)^{\frac{1}{p}} \leq \exp \left(\frac{4 C_{2}}{\bar{\Lambda}(\omega) v} \ln \frac{1}{2|\delta|}\right)^{\frac{1}{p}} \leq \frac{1}{\sqrt{2|\delta|}} .
$$

By (6.16), this choice of $\delta$ yields $|\delta|<[4(3+2 \sqrt{2})]^{-1}$, and there holds $(1-\sqrt{2|\delta|})(1+2|\delta|) \leq$ $1-\sqrt{|\delta|}$. Then, we have

$$
\sigma^{\prime}(1+2|\delta|)<\left(1-\exp \left(\frac{2 C_{\delta}}{\bar{\Lambda}(\omega) v}\right)^{-\frac{1}{p}}\right)(1+2|\delta|) \leq 1-\sqrt{|\delta|} .
$$

This implies the claimed estimate (6.20). We now proceed to estimate the right-hand side of (6.18). Using a change of variables $\tau^{\prime}=|\delta|^{-i} \tau$, we obtain

$$
\begin{aligned}
\int_{K_{4}} \zeta^{p}\left(\cdot, t_{*}\right) \phi_{-|\delta|^{i}}(\bar{u})\left(\cdot, t_{*}\right) \mathrm{d} x \\
\quad=\int_{K_{4}} \zeta^{p}\left(\cdot, t_{*}\right) \int_{0}^{\left(\bar{u}+|\delta|^{i}\right)_{+}} \frac{\mathrm{d} \tau}{\left((1-\delta)|\delta|^{i}-\tau+V\right)^{p-1}} \mathrm{~d} x \\
\quad \leq \int_{0}^{1} \frac{|\delta|^{(2-p) i}}{(1+|\delta|-\tau)^{p-1}}\left(\int_{\left.K_{4} \cap\left[\bar{u}+|\delta|^{i}\right)_{+}>\tau|\delta|^{i}\right]} \zeta^{p}\left(\cdot, t_{*}\right) \mathrm{d} x\right) \mathrm{d} \tau
\end{aligned}
$$

and this yields

$$
\begin{aligned}
\int_{K_{4}} \zeta^{p}\left(\cdot, t_{0}\right) \phi_{-|\delta|^{i}}(\bar{u})\left(\cdot, t_{0}\right) \mathrm{d} x \\
\quad \leq \int_{0}^{\tau_{*}} \frac{|\delta|^{(2-p) i}}{(1+|\delta|-\tau)^{p-1}} Y_{i} \mathrm{~d} \tau \\
\quad+\frac{C_{\delta}}{\bar{\Lambda}(\omega)} \int_{\tau_{*}}^{1} \frac{|\delta|^{(2-p) i}}{(1+|\delta|-\tau)^{p-1}}\left(\ln \frac{1+2|\delta|}{1+2|\delta|-\tau}\right)^{-p} \mathrm{~d} \tau
\end{aligned}
$$


Taking into account that $Y_{i}>v$, we obtain

$$
\int_{K_{4}} \zeta^{p}\left(\cdot, t_{0}\right) \phi_{-|\delta|^{i}}(\bar{u})\left(\cdot, t_{0}\right) \mathrm{d} x \leq|\delta|^{(2-p) i} Y_{i} G\left(Y_{i}, \delta\right)
$$

where

$$
\begin{aligned}
G\left(Y_{i}, \delta\right)= & \int_{0}^{1} \frac{\mathrm{d} \tau}{(1+|\delta|-\tau)^{p-1}} \\
& -\int_{\sigma(1+2|\delta|)}^{1}\left(1-\frac{C_{\delta}}{\nu \bar{\Lambda}(\omega)}\left(\ln \frac{1+2|\delta|}{1+2|\delta|-\tau}\right)^{-p}\right) \frac{\mathrm{d} \tau}{(1+|\delta|-\tau)^{p-1}} .
\end{aligned}
$$

Moreover, we rewrite the above estimate as

$$
\int_{K_{4}} \zeta^{p}\left(\cdot, t_{0}\right) \phi_{-|\delta|^{i}}(\bar{u})\left(\cdot, t_{0}\right) \mathrm{d} x \leq Y_{i}(1-f(\delta)) \int_{0}^{1-|\delta|} \frac{|\delta|^{(2-p) i}}{(1+|\delta|-\tau)^{p-1}} \mathrm{~d} \tau
$$

where the function $f(\delta)$ satisfies

$$
\begin{aligned}
f(\delta) & \int_{0}^{1-|\delta|} \frac{\mathrm{d} \tau}{(1+|\delta|-\tau)^{p-1}} \\
& =\int_{\sigma(1+2|\delta|)}^{1}\left(1-\frac{C_{\delta}}{v \bar{\Lambda}(\omega)}\left(\ln \frac{1+2|\delta|}{1+2|\delta|-\tau}\right)^{-p}\right) \\
& \frac{\mathrm{d} \tau}{(1+|\delta|-\tau)^{p-1}}-\int_{1-|\delta|}^{1} \frac{\mathrm{d} \tau}{(1+|\delta|-\tau)^{p-1}} .
\end{aligned}
$$

To estimate below the integral on the left-hand side of (6.18), consider the domain of integration $K_{4} \cap\left[\bar{u}>-|\delta|^{i+1}\right]$. Recalling that $V=|\delta|^{i+\frac{p}{p-1}}$, we deduce

$$
\begin{aligned}
& \left((1-\delta)|\delta|^{i}-\tau+V\right)^{p-1} \leq\left((1-\delta)|\delta|^{i}-\tau\right)^{p-1}+|\delta||\delta|^{(i+1)(p-1)} \\
& \quad \leq(1-\delta)\left((1-\delta)|\delta|^{i}-\tau\right)^{p-1}
\end{aligned}
$$

for any $\tau<|\delta|^{i}$. Then, we find that

$$
\begin{aligned}
\int_{K_{4}} \zeta^{p}\left(\cdot, t_{0}\right) \phi_{-|\delta|^{i}}(\bar{u})\left(\cdot, t_{0}\right) \mathrm{d} x & \\
& =\int_{K_{4}} \zeta^{p}\left(\cdot, t_{0}\right) \int_{0}^{\left(\bar{u}+|\delta|^{i}\right)_{+}} \frac{\mathrm{d} \tau}{\left((1-\delta)|\delta|^{i}-\tau+V\right)^{p-1}} \mathrm{~d} x \\
& \geq \int_{K_{4} \cap\left[\bar{u}>-|\delta|^{i}\right]} \zeta^{p}\left(\cdot, t_{0}\right) \int_{0}^{\left(\bar{u}+|\delta|^{i}\right)_{+}} \frac{\mathrm{d} \tau}{(1-\delta)\left((1-\delta)|\delta|^{i}-\tau\right)^{p-1}} \mathrm{~d} x \\
& \geq \int_{K_{4} \cap\left[\bar{u}>-|\delta|^{i+1}\right]} \zeta^{p}\left(\cdot, t_{0}\right) \int_{0}^{|\delta|^{i}-|\delta|^{i+1}} \frac{\mathrm{d} \tau}{(1-\delta)\left((1-\delta)|\delta|^{i}-\tau\right)^{p-1}} \mathrm{~d} x .
\end{aligned}
$$

Applying a change of variables $\tau^{\prime}=|\delta|^{-i} \tau$, we obtain

$$
\int_{K_{4}} \zeta^{p}\left(\cdot, t_{0}\right) \phi_{-|\delta|^{i}}(\bar{u})\left(\cdot, t_{0}\right) \mathrm{d} x \geq(1-\delta)^{-1}\left(Y_{i+1}-\varepsilon\right) \int_{0}^{1-|\delta|} \frac{|\delta|^{(2-p) i}}{(1+|\delta|-\tau)^{p-1}} \mathrm{~d} \tau .
$$

Combining this estimate with (6.22), we conclude that

$$
Y_{i+1} \leq(1-f(\delta))(1-\delta)^{-1} Y_{i}+\varepsilon .
$$


Finally, we need to show that $f(\delta)>\delta^{2}$. The strategy of the proof is exactly the same as in [1, page 378-379], and we include the proof here for the sake of completeness. Since $\sigma^{\prime}>\sigma$ and

$$
\frac{C_{\delta}}{v \bar{\Lambda}(\omega)}=\frac{1}{2}\left(\ln \frac{1}{1-\sigma^{\prime}}\right)^{p}
$$

It follows that

$$
\frac{C_{\delta}}{v \bar{\Lambda}(\omega)}\left(\ln \frac{1+2|\delta|}{1+2|\delta|-\tau}\right)^{-p} \leq \frac{1}{2} \text { for all } \tau \in\left(\sigma^{\prime}(1-2 \delta), 1\right) .
$$

Then, we arrive at

$$
f(\delta) \int_{0}^{1-|\delta|} \frac{\mathrm{d} \tau}{(1+|\delta|-\tau)^{p-1}} \geq \frac{1}{2} \int_{\sigma^{\prime}(1+2|\delta|)}^{1} \frac{\mathrm{d} \tau}{(1+|\delta|-\tau)^{p-1}}-\int_{1-|\delta|}^{1} \frac{\mathrm{d} \tau}{(1+|\delta|-\tau)^{p-1}} .
$$

Taking into account that $\sigma^{\prime}(1+2|\delta|)<1-\sqrt{|\delta|}$, we obtain

$$
\begin{aligned}
f(\delta) & >\frac{(|\delta|+\sqrt{|\delta|})^{2-p}-|\delta|^{2-p}-2\left((2|\delta|)^{2-p}-|\delta|^{2-p}\right)}{2\left((1+|\delta|)^{2-p}-(2|\delta|)^{2-p}\right)} \\
& >\frac{(|\delta|(1+|\delta|))^{\frac{2-p}{2}}-|\delta|^{2-p}-2\left((2|\delta|)^{2-p}-|\delta|^{2-p}\right)}{2\left((1+|\delta|)^{2-p}-(2|\delta|)^{2-p}\right)} .
\end{aligned}
$$

By (6.16), this choice of $\delta$ yields $|\delta|<e^{-64 \ln 2}$ and therefore

$$
|\delta| \leq \frac{\delta^{\prime}}{1-\delta^{\prime}}, \quad \text { where } \quad \delta^{\prime}=\inf _{z \in(1,2)}(1+32(2-z) \ln 2)^{\frac{2}{z-2}} .
$$

From this estimate, we find that

$$
(|\delta|(1+|\delta|))^{\frac{2-p}{2}}-|\delta|^{2-p} \geq 8\left((2|\delta|)^{2-p}-|\delta|^{2-p}\right) \geq 8(2-p)|\delta|^{2-p} \ln 2,
$$

since $2^{2-p}-1 \geq(2-p) \ln 2$. Taking into account that $\ln \left(1+|\delta|^{-1}\right) \leq|\delta|^{-1}$, we obtain

$$
\begin{aligned}
\left((1+|\delta|)^{2-p}\right. & \left.-(2|\delta|)^{2-p}\right)=|\delta|^{2-p} \int_{2-p}^{(2-p) \ln \left(1+|\delta|^{-1}\right)} e^{\xi} \mathrm{d} \xi \\
\leq & (2-p)|\delta|^{2-p}\left(1+|\delta|^{-1}\right) \ln \left(1+|\delta|^{-1}\right) \leq 2(2-p)|\delta|^{-p}
\end{aligned}
$$

From (6.24) and (6.25), we obtain

$$
f(\delta)>\frac{3}{8} \frac{(|\delta|(1+|\delta|))^{\frac{2-p}{2}}-|\delta|^{2-p}}{(1+|\delta|)^{2-p}-(2|\delta|)^{2-p}} \geq \frac{3 \ln 2}{2}|\delta|^{2}>\delta^{2} .
$$

Therefore, we conclude from (6.23) that $Y_{i+1} \leq(1-|\delta|) Y_{i}$, and claim (6.17) follows.

From (6.17), by iteration

$$
Y_{j+1} \leq(1-|\delta|)^{j} Y_{1} \leq(1-|\delta|)^{j}\left|K_{4}\right| .
$$

Having fixed $v \in(0,1)$, one can choose

$$
j=5+\left[\frac{\ln \frac{4^{N}}{v}}{\ln \frac{1}{1-|\delta|}}\right],
$$


where [·] denotes the integer portion of the number. For such a choice, $Y_{j+1} \leq v$, and hence,

$$
\left|\left\{x \in K_{2}: \bar{u}(x, t)>-|\delta|^{j+1}\right\}\right| \leq v\left|K_{2}\right| \text { for all } t \in\left[-2^{p}, 0\right] .
$$

Moreover, by (6.16), there exists $\gamma_{3}>2^{10}$, depending only upon the data $\left\{N, C_{0}, C_{1}\right\}$, such that

$$
\exp \left\{\left(\frac{\gamma_{4}}{v \bar{\Lambda}(\omega)}\right)^{2}\right\} \geq\left(\frac{1}{|\delta|}\right)^{2} \ln \left(\frac{4^{N}}{v}\right)
$$

We choose

$$
\tilde{m}=\tilde{m}(\nu)=\exp \left\{\left(\frac{\gamma_{4}}{v \bar{\Lambda}(\omega)}\right)^{2}\right\}
$$

Taking into account that

$$
|\delta| \leq \ln \frac{1}{1-|\delta|} \leq 2|\delta| \text { and } \ln \frac{1}{|\delta|} \leq \frac{1}{\sqrt{|\delta|}},
$$

the choice (6.26) guarantees that $2^{-\tilde{m}} \leq|\delta|^{j+1}$. For such a choice of $\tilde{m}$, we obtain the desired estimate (6.11). Finally, if $Y_{i_{0}} \leq v$ for some $i_{0} \in\{1,2, \ldots, j\}$, then the estimate (6.11) also holds for the same choice of $\tilde{m}$ as in (6.26). This concludes the proof of the lemma.

From (6.9), (6.14) and (6.26), we remark that $\tilde{m}$ can be chosen independent of $m_{2}$. Transforming back to the original function $u$ and original variables $(x, t)$, we obtain an estimate for the measure of the level sets

$$
\left|\left\{x \in \bar{x}+K_{4 c_{2} R}: u(x, t)>\mu_{+}-\frac{\omega}{2^{m_{2}+\tilde{n}+\tilde{m}-1}}\right\}\right|<\nu\left|K_{4 c_{2} R}\right|,
$$

for all $\tilde{t}-2^{-p}\left(\tilde{t}-t_{0}\right)<t<\tilde{t}$. With the help of this estimate, we can now prove a DeGiorgitype lemma.

Lemma 6.5 Let $\tilde{n}>1$ be the constant chosen according to (6.4). Then, there exists a constant $\tilde{m}>1$ depending only upon the data and $v_{0}$, and a time level $t_{\omega} \in\left(t_{0}, \tilde{t}\right)$ such that

$$
u(x, t)<\mu_{+}-\frac{\omega}{2^{m_{2}+\tilde{n}+\tilde{m}}} \text { a.e. in }(\bar{x}, \tilde{t})+Q\left(\frac{\tilde{t}-t_{\omega}}{2^{p}}, 2 c_{2} R\right) .
$$

Proof Without loss of generality, we may assume $(\bar{x}, \tilde{t})=(0,0)$. For $v \in(0,1)$ to be determined later, we take $\tilde{m}=\tilde{m}(v)>1$ according to (6.26). For $n=0,1,2, \ldots$, set

$$
\begin{aligned}
R_{n} & =2 c_{2} R+\frac{2 c_{2} R}{2^{n}}, \quad k_{n}=\mu_{+}-\frac{\omega}{2^{m_{2}+\tilde{n}+\tilde{m}}}-\frac{\omega}{2^{m_{2}+\tilde{n}+\tilde{m}+n}} \text { and } \\
Q_{n} & =Q\left(\frac{\tilde{t}-t_{\omega}}{2^{p}}+\frac{\tilde{t}-t_{\omega}}{2^{p+p n}}, R_{n}\right)
\end{aligned}
$$

where the time level $t_{\omega}$ is taken as

$$
t_{\omega}=\tilde{t}-\left(\frac{\nu_{0}}{4}\right)\left(\frac{1}{2^{\tilde{n}+\tilde{m}}}\right)^{2-p}\left(\frac{\omega}{2^{m_{2}}}\right)^{(p-1)(p-2)} R^{p} .
$$

From (6.4), (6.9), (6.14) and (6.26), we observe that

$$
\tilde{m}(v) \geq \tilde{m}(1)>\tilde{n}>(p-1) \tilde{n}
$$


for any $v \in(0,1)$. Therefore, we conclude from (6.3) that $t_{\omega} \in\left(t_{0}, \tilde{t}\right)$. Take piecewise smooth cutoff functions $\zeta_{n}$ in $Q_{n}$ such that $0 \leq \zeta_{n} \leq 1, \zeta_{n} \equiv 1$ in $Q_{n+1}, \zeta_{n} \equiv 0$ on $\partial_{P} Q_{n}$, $\left|D \zeta_{n}\right| \leq 2^{n} /\left(c_{2} R\right)$ and $0<\partial_{t} \zeta_{n} \leq 2^{p+1} 2^{p n} /\left(\tilde{t}-t_{\omega}\right)$. Write the energy estimates (3.3) for the truncated functions $\left(u-k_{n}\right)_{+} \zeta_{n}^{p}$ over the cylinders $Q_{n}$. Taking into account that

$$
U\left(K_{R_{n}},-2^{-p}\left(\tilde{t}-t_{\omega}\right)-2^{-p-p n}\left(\tilde{t}-t_{\omega}\right), 0,\left(u-k_{n}\right)_{+} \zeta_{n}^{p}\right)=0,
$$

we deduce

$$
\begin{aligned}
& \lim _{-2^{-p}\left(\tilde{t}-t_{\omega}\right)-2^{-p-p n}\left(\tilde{t}-t_{\omega}\right)<t<0} \int_{K_{R_{n} \times\{t\}}}\left(u-k_{n}\right)_{+}^{2} \zeta_{n}^{p} \mathrm{~d} x \\
& +\iint_{Q_{n}}\left|D\left(u-k_{n}\right)_{+} \zeta_{n}\right|^{p} \mathrm{~d} x \mathrm{~d} t \\
& \leq 2^{2 n+6} \gamma_{1} v_{0}^{-1} R^{-p}\left(\frac{\omega}{2^{m_{2}}}\right)^{(3-p) p}\left(\frac{1}{2^{\tilde{m}+\tilde{n}}}\right)^{p} \\
& \iint_{Q_{n}} \chi_{\left[\left(u-k_{n}\right)_{+}>0\right]} \mathrm{d} x \mathrm{~d} t .
\end{aligned}
$$

At this point, we set

$$
A_{n}=\iint_{Q_{n}} \chi_{\left[\left(u-k_{n}\right)_{+}>0\right]} \mathrm{d} x \mathrm{~d} t \text { and } Y_{n}=\frac{A_{n}}{\left|Q_{n}\right|} .
$$

Applying the parabolic Sobolev inequality (2.7), we obtain

$$
\begin{aligned}
& \iint_{Q_{n}}\left|\left(u-k_{n}\right)_{+}\right|^{p\left(1+\frac{2}{N}\right)} \zeta_{n}^{p\left(1+\frac{2}{N}\right)} \mathrm{d} x \mathrm{~d} t \\
& \quad \leq \gamma\left(\operatorname{ess~sup}_{-2^{-p}\left(\tilde{t}-t_{\omega}\right)-2^{-p-p n}\left(\tilde{t}-t_{\omega}\right)<t<0} \int_{K_{R_{n}} \times\{t\}}\left(u-k_{n}\right)_{+}^{2} \zeta_{n}^{2} \mathrm{~d} x\right)^{\frac{p}{N}} \\
& \quad \iint_{Q_{n}}\left|D\left(u-k_{n}\right)_{+} \zeta_{n}\right|^{p} \mathrm{~d} x \mathrm{~d} t \\
& \leq \gamma_{5} v_{0}^{-\left(1+\frac{p}{N}\right)} 2^{2 n\left(1+\frac{p}{N}\right)} R^{-p\left(1+\frac{p}{N}\right)}\left(\frac{\omega}{2^{m_{2}}}\right)^{(3-p) p\left(1+\frac{p}{N}\right)} \\
& \quad\left(\frac{1}{2^{\tilde{m}+\tilde{n}}}\right)^{p\left(1+\frac{p}{N}\right)} A_{n}^{1+\frac{p}{N}}
\end{aligned}
$$

for a constant $\gamma_{5}$ depending only upon the data. The integral on the left-hand side is estimated below by

$$
\begin{gathered}
\iint_{Q_{n}}\left|\left(\bar{u}-k_{n}\right)_{+}\right|^{p\left(1+\frac{2}{N}\right)} \zeta_{n}^{p\left(1+\frac{2}{N}\right)} \mathrm{d} x \mathrm{~d} t \geq\left(k_{n+1}-k_{n}\right)^{p \frac{N+2}{N}} A_{n+1} \\
\geq\left(\frac{\omega}{2^{m_{2}+\tilde{n}+\tilde{m}+n+1}}\right)^{p \frac{N+2}{N}} A_{n+1} .
\end{gathered}
$$

Combining the estimates above and keeping in mind

$$
\left|Q_{n}\right| \leq 4^{N} L_{2}^{N} \nu_{0}\left(\frac{\omega}{2^{m_{2}}}\right)^{(p-2) N+(p-1)(p-2)}\left(\frac{1}{2^{\tilde{n}+\tilde{m}}}\right)^{2-p} R^{N+p},
$$


we infer that

$$
Y_{n+1} \leq \gamma_{6} v_{0}^{-1} 2^{4 n\left(1+\frac{2}{N}\right)} Y_{n}^{1+\frac{p}{N}} \leq \gamma_{6} v_{0}^{-1} 2^{4 n\left(1+\frac{2}{N}\right)} Y_{n}^{1+\frac{2}{N}},
$$

for a constant $\gamma_{6}$ depending only upon the data. At this point, we set

$$
v_{2}=2^{-N(N+2)} \gamma_{6}^{-\frac{N}{2}} \nu_{0}^{\frac{N}{2}} .
$$

Choose $v=v_{2}$ in Lemma 6.4, and hence $\tilde{m}=\tilde{m}\left(v_{2}\right)$ from this and (6.26). Moreover, we conclude from (6.27) that $Y_{0} \leq \nu_{2}$. By the lemma on fast geometric convergence of sequences, we infer that $Y_{n} \rightarrow 0$, as $n \rightarrow \infty$, which proves the lemma.

\subsection{Expansion of positivity in the time variable}

The aim of this subsection is to establish a DeGiorgi-type result similar to that of Lemma 6.5. To start with, we determine the constant $m_{2}$ in terms of the data and $\omega$. Let $\tilde{n}$ and $\nu_{2}$ be the constants determined by (6.4) and (6.29), respectively. In (6.26), take $v=v_{2}$, and choose $\tilde{m}=\tilde{m}\left(v_{2}\right)$. At this point, we choose $m_{2}>2$ such that

$$
m_{2} p=m_{2}+\tilde{n}+\tilde{m}+10 \text { i. e., } \quad m_{2}=\frac{\tilde{n}+\tilde{m}+10}{p-1},
$$

and hence $m_{1}=m_{2} p /(p-1)$. This determines the precise formulation of the intrinsic parabolic cylinders. Next, we consider some geometric properties of these cylinders.

Henceforth, we assume that $p>\frac{1+\sqrt{5}}{2}$ and set $\kappa=\frac{1}{2}\left(p-1-\frac{1}{p}\right)>0$. Moreover, we will need the following assumption:

$$
R \leq \min \left\{\left(\frac{1}{8 L_{2}}\right)^{\frac{1}{\kappa}},\left(\frac{1}{16}\right)^{\frac{1}{(p-1)^{2}}}\right\} .
$$

In the case $2^{-m_{2}} \omega \geq R$. Keeping in mind $L_{1}=2$, we conclude from (6.31) that $Q\left(8 c_{1} R^{p}, 8 c_{2} R\right) \subset \bar{Q}$. While in the case $2^{-m_{2}} \omega \leq R$ we conclude from (5.4), (6.4), (6.9), (6.14), (6.26), (6.29) and (6.30) that

$$
\exp \left(-\exp \left(\exp \frac{\gamma_{8}}{\omega^{\gamma /}}\right)\right) \leq R
$$

for some constants $\gamma_{7}$ and $\gamma_{8}$ depending only upon the data. From this inequality, we obtain a decay estimate for $\omega$ as follows:

$$
\omega \leq\left(\frac{\gamma_{8}}{\ln \ln \ln \frac{1}{R}}\right)^{\frac{1}{\gamma_{7}}} .
$$

We now turn our attention to the case $Q\left(8 c_{1} R^{p}, 8 c_{2} R\right) \subset \bar{Q}$. In this case, we have already established the estimate (6.28). Before proceeding further, let us remark that this estimate implies

$$
u(x, \tilde{t})<\mu_{+}-\frac{\omega}{2^{m_{2}+\tilde{n}+\tilde{m}}} \text { a.e. } x \in K_{c_{2} R} .
$$

The next lemma addresses the expansion of positivity in the time variable, starting from $\tilde{t}$. 
Lemma 6.6 For any $v \in(0,1)$, there exist a constant $\gamma_{9}$, depending only upon the data, and a time level $t^{(0)}=\tilde{t}+\gamma_{9} v \omega^{(1-p)(2-p)} R^{p}$ such that

$$
\left|\left\{x \in K_{\frac{1}{2} c_{2} R}: u(x, t)>\mu_{+}-\frac{\omega}{2^{m_{2} p-1}}\right\}\right|<v\left|K_{\frac{1}{2} c_{2} R}\right|
$$

for any $t \in\left(\tilde{t}, t^{(0)}\right)$.

Proof Set $k=\mu_{+}-\frac{\omega}{2^{m_{2} p^{p-4}}}$ and $c=\frac{\omega}{2^{m_{2} p^{p-1}}}$. We consider the logarithmic function

$$
\psi^{+}=\ln ^{+}\left(\frac{\frac{\omega}{2^{m_{2} p-4}}}{\frac{\omega}{2^{m_{2} p-4}}-(u-k)_{+}+c}\right) .
$$

Then, we have $\psi^{+} \leq 8 \ln 2$ and

$$
\left[\left(\psi^{+}\right)^{\prime}\right]^{2-p} \leq\left(\frac{1}{c}\right)^{2-p} \leq\left(\frac{\omega}{2^{m_{2} p-1}}\right)^{p-2} .
$$

Choose a piecewise smooth cutoff function $\zeta(x)$, defined in $K_{c_{2} R}$ and satisfying $0 \leq \zeta \leq 1$ in $K_{c_{2} R}, \zeta \equiv 1$ in $K_{\frac{1}{2} c_{2} R}$ and $|D \zeta| \leq 2\left(c_{2} R\right)^{-1}$. Since $k>\mu_{+}-\frac{\omega}{4}$, it is easy to check that $U\left(K_{c_{2} R}, \tilde{t}, t^{(0)}, 2 \psi^{+}\left(\psi^{+}\right)^{\prime} \zeta^{p}\right)=0$. Then, we obtain from (3.4) the logarithmic estimate

$$
\begin{gathered}
\underset{\tilde{t}<t<t^{(0)}}{\operatorname{ess} \sup _{K_{c_{2} R} \times\{t\}}} \int_{\left.K^{+}\right)^{2} \zeta^{p} \mathrm{~d} x \leq \int_{K_{c_{2} R} \times\{\tilde{t}\}}\left(\psi^{+}\right)^{2} \zeta^{p} \mathrm{~d} x} \\
+\gamma_{2} \int_{\tilde{t}}^{t^{(0)}} \int_{K_{c_{2} R}} \psi^{+}\left|\left(\psi^{+}\right)^{\prime}\right|^{2-p}|D \zeta|^{p} \mathrm{~d} x \mathrm{~d} t
\end{gathered}
$$

From (6.33), we observe that $\int_{K_{c_{2} R} \times\{\tilde{t}\}}\left(\psi^{+}\right)^{2} \zeta^{p} \mathrm{~d} x=0$ and there holds

$$
\begin{aligned}
\operatorname{ess~sup}_{\tilde{t}<t<t^{(0)}} \int_{K_{c_{2} R} \times\{t\}} & \left(\psi^{+}\right)^{2} \zeta^{p} \mathrm{~d} x \leq \gamma_{2} \int_{\tilde{t}}^{t^{(0)}} \int_{K_{c_{2} R}} \psi^{+}\left|\left(\psi^{+}\right)^{\prime}\right|^{2-p}|D \zeta|^{p} \mathrm{~d} x \mathrm{~d} t \\
& \leq 2^{N+5}(\ln 2) \gamma_{2} L_{2}^{-1} \omega^{(1-p)(p-2)} R^{-p}\left(t^{(0)}-\tilde{t}\right)\left|K_{\frac{1}{2} c_{2} R}\right| \leq v\left|K_{\frac{1}{2} c_{2} R}\right|
\end{aligned}
$$

provided

$$
\gamma_{9}=2^{-N-5} \gamma_{2}^{-1} L_{2}
$$

To estimate below the integral on the left-hand side, we consider a smaller set defined by

$$
S=\left\{x \in K_{\frac{1}{2} c_{2} R}: u(x, t)>\mu_{+}-\frac{\omega}{2^{m_{2} p-1}}\right\} \subset K_{\frac{1}{2} c_{2} R} .
$$

On such a set, $\zeta \equiv 1$ and $\psi^{+} \geq 2 \ln 2>1$. Combining this inequality with (6.35), we obtain (6.34), which proves the lemma.

With the help of this lemma, we can now prove the following DeGiorgi-type result.

Lemma 6.7 There exists a constant $\nu_{3} \in(0,1)$ depending only upon the data such that

$$
u(x, t)<\mu_{+}-\frac{\omega}{2^{m_{2} p}} \text { a.e. in } K_{\frac{1}{4} c_{2} R} \times\left(\tilde{t}, t^{(0)}\right],
$$

where $t^{(0)}=\tilde{t}+\gamma_{9} \nu_{3} \omega^{(1-p)(2-p)} R^{p}$. 
Proof Let $t^{(0)}=\tilde{t}+\gamma_{9} v \omega^{(1-p)(2-p)} R^{p}$, where $v \in(0,1)$ is to be determined. Consider two decreasing sequences of real numbers

$$
R_{n}=c_{2} \frac{R}{4}+c_{2} \frac{R}{4^{n+1}} \quad \text { and } \quad k_{n}=\mu_{+}-\frac{\omega}{2^{m_{2} p}}-\frac{\omega}{2^{m_{2} p+n}} \quad n=0,1,2, \ldots
$$

We set $Q_{n}=K_{R_{n}} \times\left(\tilde{t}, t^{(0)}\right]$. Take piecewise smooth cutoff functions $\zeta_{n}(x)$ in $K_{R_{n}}$, such that $0 \leq \zeta_{n} \leq 1$ in $K_{R_{n}}, \zeta_{n} \equiv 1$ in $K_{R_{n+1}}$ and $\left|D \zeta_{n}\right| \leq 4^{n+2}\left(c_{2} R\right)^{-1}$. Write the energy estimates (3.3) for the truncated functions $\left(u-k_{n}\right)_{+}$over the cylinders $Q_{n}$. Taking into account that

$$
\partial_{t} \zeta_{n} \equiv 0 \quad \text { and } \quad U\left(K_{R_{n}}, \tilde{t}, t^{(0)},\left(u-k_{n}\right)_{+} \zeta_{n}^{p}\right)=0,
$$

we obtain

$$
\begin{aligned}
& \underset{\tilde{t}<t<t^{(0)}}{\operatorname{ess} \sup _{K_{R_{n}} \times\{t\}}} \int\left(u-k_{n}\right)_{+}^{2} \zeta_{n}^{p} \mathrm{~d} x+\iint_{Q_{n}}\left|D\left(u-k_{n}\right)_{+} \zeta_{n}\right|^{p} \mathrm{~d} x \mathrm{~d} t \\
& \quad \leq \gamma_{1} \iint_{Q_{n}}\left(u-k_{n}\right)_{+}^{p}\left|D \zeta_{n}\right|^{p} \mathrm{~d} x \mathrm{~d} t \leq \frac{4^{n+3} \gamma_{1} \omega^{p(3-p)}}{L_{2}^{p} 2^{2 m_{2} p} R^{p}} \iint_{Q_{n}} \chi_{\left[\left(u-k_{n}\right)_{+}>0\right]} \mathrm{d} x \mathrm{~d} t .
\end{aligned}
$$

Set

$$
A_{n}=\iint_{Q_{n}} \chi_{\left[\left(u-k_{n}\right)_{+}>0\right]} \mathrm{d} x \mathrm{~d} t \quad \text { and } \quad Y_{n}=\frac{A_{n}}{\left|Q_{n}\right|} .
$$

Applying the parabolic Sobolev inequality (2.7), we obtain

$$
\begin{aligned}
& \iint_{Q_{n}}\left|\left(u-k_{n}\right)_{+} \zeta_{n}\right|^{p \frac{N+2}{N}} \mathrm{~d} x \mathrm{~d} t \\
& \quad \leq \gamma\left({\operatorname{ess} \sup _{\tilde{t}<t<t^{(0)}}}_{K_{R_{n}} \times\{t\}}\left(u-k_{n}\right)_{+}^{2} \zeta_{n}^{2} \mathrm{~d} x\right)^{\frac{p}{N}} \iint_{Q_{n}}\left|D\left(u-k_{n}\right)_{+} \zeta_{n}\right|^{p} \mathrm{~d} x \mathrm{~d} t \\
& \quad \leq \gamma_{10} 4^{2 n}\left(\omega^{(3-p) p} 2^{-2 p m_{2}}\right)^{1+\frac{p}{N}} R^{-p\left(1+\frac{p}{N}\right)} A_{n}^{1+\frac{p}{N}},
\end{aligned}
$$

for a constant $\gamma_{10}$ depending only upon the data. The integral on the left-hand side is estimated below by

$$
\iint_{Q_{n}}\left|\left(u-k_{n}\right)_{+} \zeta_{n}\right|^{p \frac{N+2}{N}} \mathrm{~d} x \mathrm{~d} t \geq\left(k_{n+1}-k_{n}\right)^{p \frac{N+2}{N}} A_{n+1} \geq\left(\frac{\omega}{2^{m_{2}} p+n+1}\right)^{p \frac{N+2}{N}} A_{n+1} .
$$

Combining (6.37) and (6.38), we have

$$
A_{n+1} \leq \gamma_{10} 2^{\frac{2(N+2)}{N}} 4^{6 n} \omega^{-\frac{p^{3}}{N}+\left(\frac{3}{N}-1\right) p^{2}+2\left(1-\frac{1}{N}\right) p} 2^{m_{2} p(p-2)} R^{-p\left(1+\frac{p}{N}\right)} A_{n}^{1+\frac{p}{N}} .
$$

Taking into account that

$$
\left|Q_{n}\right|=\gamma_{9} v L_{2}^{N}\left(\frac{\omega}{2^{m_{2}}}\right)^{N(p-2)}\left(\frac{1}{4}+\frac{1}{4^{n+1}}\right)^{N} \omega^{(1-p)(2-p)} R^{N+p}
$$

and $v<1$, we obtain

$$
Y_{n+1} \leq \gamma_{11} 4^{6 n} v^{\frac{p}{N}} Y_{n}^{1+\frac{p}{N}} \leq \gamma_{11} 4^{6 n} Y_{n}^{1+\frac{2}{N}}
$$


for a constant $\gamma_{11}$ depending only upon the data. At this point, we set

$$
v_{3}=\gamma_{11}^{-\frac{N}{2}} 4^{-\frac{3 N^{2}}{2}} \text {. }
$$

We now choose $v=v_{3}$. By Lemma 6.6, $Y_{0} \leq v_{3}$. Applying the lemma on fast geometric convergence of sequences, we deduce $Y_{n} \rightarrow 0$ as $n \rightarrow \infty$, which proves the lemma.

\subsection{Iterative arguments: time propagation of positivity from $t^{(0)}$ to $t^{(1)}$}

In this subsection, we first remark that the time level $t^{(0)}$ could be lower than $\bar{t}$. Therefore, the estimate (6.36) is insufficient for the proof. We have to use an iterative argument to obtain an estimate similar to (6.36) in the cylinder with a larger time interval.

The starting point is a space propagation of positivity similar to Lemma 6.4. Starting from (6.36), we set $R^{(0)}=\frac{1}{4} c_{2} R$ and introduce the change of variables

$$
x^{\prime}=\frac{x}{20 R^{(0)}} \quad \text { and } \quad t^{\prime}=\frac{t-t^{(0)}}{t^{(0)}-\tilde{t}} .
$$

This transformation maps $K_{R^{(0)}} \times\left(\tilde{t}, t^{(0)}\right] \rightarrow K_{\frac{1}{20}} \times(-1,0]$ and $K_{20 R^{(0)}} \times\left(\tilde{t}, t^{(0)}\right] \rightarrow$ $K_{1} \times(-1,0]$. Moreover, we set the new functions

$$
\tilde{u}\left(x^{\prime}, t^{\prime}\right)=u(x, t) \text { and } \bar{u}\left(x^{\prime}, t^{\prime}\right)=\left(\tilde{u}\left(x^{\prime}, t^{\prime}\right)-\mu_{+}\right)\left(\frac{2^{m_{2} p}}{\omega}\right) .
$$

With these notations, the estimate (6.36) implies

$$
\left|\left\{x^{\prime} \in K_{1}: \bar{u}\left(x^{\prime}, t^{\prime}\right)<-1\right\}\right| \geq\left|K_{\frac{1}{20}}\right|=20^{-N}
$$

for all $t^{\prime} \in(-1,0]$. On the other hand, we set $\tilde{w}\left(x^{\prime}, t^{\prime}\right)=w(x, t)$, and $v(x, t)$ in (3.1) can be written in the new variables as

$$
\tilde{v}\left(x^{\prime}, t^{\prime}\right)= \begin{cases}\bar{v}, & \text { on }\left\{\bar{u}<-\mu_{+} \frac{2^{m_{2} p}}{\omega}\right\}, \\ -\tilde{w}\left(x^{\prime}, t^{\prime}\right), & \text { on }\left\{\bar{u}=-\mu_{+} \frac{2^{m_{2} p}}{\omega}\right\} .\end{cases}
$$

We rewrite the weak form (3.1) in terms of the new variables and new functions

$$
\begin{aligned}
& -\left.\frac{2^{m_{2} p}}{\omega} \int_{K_{1}} \tilde{v}\left(\cdot, t^{\prime}\right) \chi_{\left\{\bar{u} \leq-\mu_{+} \frac{2^{m_{2} p}}{\omega}\right\}} \varphi\left(\cdot, t^{\prime}\right) \mathrm{d} x^{\prime}\right|_{t^{\prime}=t_{1}} ^{t_{2}} \\
& \frac{2^{m_{2} p}}{\omega} \int_{t_{1}}^{t_{2}} \int_{K_{1}} \tilde{v} \chi_{\left\{\bar{u} \leq-\mu_{+} \frac{2^{m_{2} p}}{\omega}\right\}} \frac{\partial \varphi}{\partial t^{\prime}} \mathrm{d} x^{\prime} \mathrm{d} t^{\prime} \\
& \quad+\int_{t_{1}}^{t_{2}} \int_{K_{1}}\left(\varphi \frac{\partial \bar{u}}{\partial t^{\prime}}+\bar{A}\left(x^{\prime}, t^{\prime}, \bar{u}, D \bar{u}\right) \cdot D \varphi\right) \mathrm{d} x^{\prime} \mathrm{d} t^{\prime}=0
\end{aligned}
$$

for any $\varphi \in W_{p}\left(Q_{1}\right)$ and $\left[t_{1}, t_{2}\right] \subset(-1,0]$. We observe that the vector field $\bar{A}$ satisfies the structure condition

$$
\left\{\begin{array}{l}
\bar{A}\left(x^{\prime}, t^{\prime}, \bar{u}, D \bar{u}\right) \cdot D \bar{u} \geq C_{0} \Lambda_{1}|D \bar{u}|^{p} \\
\left|\bar{A}\left(x^{\prime}, t^{\prime}, \bar{u}, D \bar{u}\right)\right| \leq C_{1} \Lambda_{1}|D \bar{u}|^{p-1}
\end{array}\right.
$$

where

$$
\Lambda_{1}=\left(\frac{\omega}{2^{m_{2} p}}\right)^{p-2} \frac{t^{(0)}-\tilde{t}}{\left(20 R^{(0)}\right)^{p}}
$$


Recalling that $t^{(0)}=\tilde{t}+\gamma_{9} v_{3} \omega^{(1-p)(2-p)} R^{p}$ and $R^{(0)}=\frac{1}{4} c_{2} R$, we deduce

$$
\Lambda_{1}=\frac{\gamma_{9} \nu_{3}}{\left(5 L_{2}\right)^{p}} .
$$

The constant $\Lambda_{1}$ depends only upon the data. To simplify notation, we continue to write $(x, t)$ for the new variables. In the same fashion as in the proof of Lemma 6.3, we conclude that the truncated functions $(\bar{u}-k)_{+}$are subsolutions to parabolic equations. To be more precise, we have

$$
\int_{t_{1}}^{t_{2}} \int_{K_{1}} \varphi \frac{\partial(\bar{u}-k)_{+}}{\partial t}+\bar{A}\left(x, t, k+(\bar{u}-k)_{+}, D(\bar{u}-k)_{+}\right) \cdot D \varphi \mathrm{d} x \mathrm{~d} t \leq 0
$$

for all $k \in(-1,0), t_{1}, t_{2} \in(-1,0)$ and all nonnegative $\varphi \in W_{p}\left(Q_{1}\right)$.

Lemma 6.8 Suppose that $\frac{3}{2} \leq p<2$. For any $v \in(0,1)$, there exists $\bar{m}>1$ depending only upon the data and $v$, such that

$$
\left|\left\{x \in K_{\frac{3}{4}}: \bar{u}(x, t) \geq-2^{-\bar{m}}\right\}\right| \leq v\left|K_{\frac{3}{4}}\right|
$$

for all $t \in\left(-\left(\frac{3}{4}\right)^{p}, 0\right]$.

Proof We proceed similarly as in the proof of Lemma 6.4. To this end, we introduce the auxiliary functions

$$
\phi_{k}(\bar{u})=\int_{0}^{(\bar{u}-k)_{+}} \frac{\mathrm{d} \tau}{(-(1-\delta) k-\tau+V)^{p-1}}
$$

and

$$
\psi_{k}(\bar{u})=\ln \left(\frac{-(1-\delta) k+V}{-k(1-\delta)-(\bar{u}-k)_{+}+V}\right)
$$

where $k, \delta \in\left(-\frac{1}{8}, 0\right)$ and $V=(-k)(-\delta)^{\frac{p}{p-1}}$. Take a piecewise smooth cutoff function $\zeta=\zeta_{1}(x) \zeta_{2}(t)$ in $Q_{1}$, such that $0 \leq \zeta \leq 1$ in $Q_{1}, \zeta \equiv 1$ in $Q_{\frac{3}{4}}, \zeta \equiv 0$ on $\partial_{P} Q_{1},\left|D \zeta_{1}\right| \leq 4$, $0 \leq \partial_{t} \zeta_{2} \leq 4^{p}$ and the sets $\left\{x \in K_{1}: \zeta_{1}(x)>-k\right\}$ are convex for all $k \in\left(-\frac{1}{8}, 0\right)$. In the weak formulation (6.44), take the test function

$$
\varphi=\frac{\zeta^{p}}{\left(-(1-\delta) k-(\bar{u}-k)_{+}+V\right)^{p-1}} .
$$

This yields

$$
\left.\int_{K_{1}} \zeta^{p} \phi_{k}(\bar{u}) \mathrm{d} x\right|_{t=t_{1}} ^{t_{2}}+\frac{1}{4} C_{0} \Lambda_{1} \int_{t_{1}}^{t_{2}} \int_{K_{1}} \zeta^{p}\left|D \psi_{k}(\bar{u})\right|^{p} \mathrm{~d} x \mathrm{~d} t \leq 16 C_{2}\left(t_{2}-t_{1}\right) \ln \frac{1-\delta}{-\delta}
$$

for any $\left[t_{1}, t_{2}\right] \in(-1,0]$. Next, we observe from (6.40) that

$$
K_{\frac{1}{20}} \subset\left\{x \in K_{1}: \bar{u}(x, t)<-1\right\} \subset\left\{x \in K_{1}: \phi_{k}(\bar{u})=0\right\} \cap\left\{x \in K_{1}: \zeta_{1}(x)=1\right\},
$$

for any $t \in(-1,0]$. Applying the Sobolev inequality (2.6) slicewise, we obtain

$$
\int_{K_{1}} \zeta_{1}^{p} \psi_{k}(\bar{u})^{p} \mathrm{~d} x \leq 20^{N-1} \gamma \int_{K_{1}} \zeta_{1}^{p}\left|D \psi_{k}(\bar{u})\right|^{p} \mathrm{~d} x, \quad \forall t \in(-1,0] .
$$


With the same argument as in the proof of Lemma 6.4, we derive the estimate

$$
\frac{\mathrm{d}^{-}}{\mathrm{d} t} \int_{K_{1}} \zeta^{p} \phi_{k}(\bar{u}) \mathrm{d} x+\Lambda_{2} \int_{K_{1}} \zeta^{p} \psi_{k}(\bar{u})^{p} \mathrm{~d} x \leq 16 C_{2} \ln \frac{1-\delta}{-\delta}, \quad \forall t \in(-1,0],
$$

where the constant $\Lambda_{2}$ depends only upon the data. At this stage, we follow the proof of Lemma 6.4 to conclude that there exists a constant $\gamma^{\prime}$ depending only on the data, such that the following holds. If we choose

$$
\bar{m}=\bar{m}(v)=\exp \left\{\left(\frac{\gamma^{\prime}}{v \Lambda_{2}}\right)^{2}\right\},
$$

then the estimate (6.45) follows. Moreover, we observe that the constant $\bar{m}$ depends only upon the data.

Lemma 6.9 There exist a constant $\bar{m}>1$, depending only upon the data and $p$, and a time level $t_{\omega}^{\prime} \in\left(-\left(\frac{3}{4}\right)^{p}, 0\right)$ such that

$$
\bar{u}(x, t)<-\frac{1}{2^{\bar{m}+1}} \text { a.e. in } Q\left(-\frac{t_{\omega}^{\prime}}{2^{p}}, \frac{1}{2}\right),
$$

and the constant $\bar{m}$ is stable as $p \rightarrow 2$.

Proof For $v \in(0,1)$ to be determined later, we take $\bar{m}>1$ according to (6.46). For $n=$ $0,1,2, \ldots$, set

$$
R_{n}=\frac{1}{2}+\frac{1}{2^{n+2}}, \quad k_{n}=-\frac{1}{2^{\bar{m}+1}}-\frac{1}{2^{\bar{m}+n+1}} \quad \text { and } \quad Q_{n}=Q\left(\frac{-t_{\omega}^{\prime}}{2^{p}}+\frac{-t_{\omega}^{\prime}}{2^{p+p n}}, R_{n}\right),
$$

where $t_{\omega}^{\prime}=-2^{(\bar{m}+1)(p-2)}\left(\frac{3}{4}\right)^{p}$. Take piecewise smooth cutoff functions $\zeta_{n}$ in $Q_{n}$, such that $0 \leq \zeta_{n} \leq 1, \zeta_{n} \equiv 1$ in $Q_{n+1}, \zeta_{n} \equiv 0$ on $\partial_{P} Q_{n},\left|D \zeta_{n}\right| \leq 2^{n+3}$ and $0<\partial_{t} \zeta_{n} \leq$ $2^{p+p(n+1)}\left(-t_{\omega}^{\prime}\right)^{-1}$.

In the weak formulation (6.41), take the test function $\varphi=\left(\bar{u}-k_{n}\right)_{+} \zeta_{n}^{p}$. Observe that the first two terms vanish. By a standard argument, we derive a Caccioppoli estimate for $\left(\bar{u}-k_{n}\right)_{+}$over $Q_{n}$ as follows:

$$
\begin{aligned}
& \operatorname{ess~sup}_{2^{-p} t_{\omega}^{\prime}+2^{-p-p n} t_{\omega}^{\prime}<t<0} \frac{1}{2} \int_{K_{R_{n} \times\{t\}}}\left(\bar{u}-k_{n}\right)_{+}^{2} \zeta_{n}^{p} \mathrm{~d} x \\
& +\frac{1}{2} C_{0} \Lambda_{1} \iint_{Q_{n}}\left|D\left(\bar{u}-k_{n}\right)_{+}\right|^{p} \zeta_{n}^{p} \mathrm{~d} x \mathrm{~d} t \\
& \leq 8 C_{0}\left(\frac{C_{1}}{C_{0}}\right)^{2} \Lambda_{1} \iint_{Q_{n}}\left(\bar{u}-k_{n}\right)_{+}^{p}\left|D \zeta_{n}\right|^{p} \mathrm{~d} x \mathrm{~d} t \\
& +\frac{1}{2} \iint_{Q_{n}}\left(\bar{u}-k_{n}\right)_{+}^{2} \frac{\partial \zeta_{n}^{p}}{\partial t} \mathrm{~d} x \mathrm{~d} t .
\end{aligned}
$$

Applying the parabolic Sobolev inequality (2.7), we obtain

$$
\begin{aligned}
\iint_{Q_{n}} & \left|\left(\bar{u}-k_{n}\right)_{+}\right|^{p\left(1+\frac{2}{N}\right)} \zeta_{n}^{p\left(1+\frac{2}{N}\right)} \mathrm{d} x \mathrm{~d} t \\
& \leq \gamma\left(\operatorname{ess~sup}_{2^{-p} t_{\omega}^{\prime}+2^{-p-p n} t_{\omega}^{\prime}<t<0} \int_{K_{R_{n}} \times\{t\}}\left(\bar{u}-k_{n}\right)_{+}^{2} \zeta_{n}^{2} \mathrm{~d} x\right)^{\frac{p}{N}}
\end{aligned}
$$




$$
\begin{aligned}
& \iint_{Q_{n}}\left|D\left(\bar{u}-k_{n}\right)_{+} \zeta_{n}\right|^{p} \mathrm{~d} x \mathrm{~d} t \\
\leq & C_{3} 2^{p n\left(1+\frac{p}{N}\right)}\left(\frac{1}{2^{\tilde{m}}}\right)^{p+\frac{p^{2}}{N}}\left(\iint_{Q_{n}} \chi_{\left[\left(\bar{u}-k_{n}\right)_{+}>0\right]} \mathrm{d} x \mathrm{~d} t\right)^{1+\frac{p}{N}},
\end{aligned}
$$

for a constant $C_{3}$ depending only upon the data. At this point, we set

$$
A_{n}=\iint_{Q_{n}} \chi_{\left[\left(\bar{u}-k_{n}\right)_{+}>0\right]} \mathrm{d} x \mathrm{~d} t \quad \text { and } \quad Y_{n}=\frac{A_{n}}{\left|Q_{n}\right|} .
$$

The left-hand side of (6.48) is estimated below by

$$
\iint_{Q_{n}}\left|\left(\bar{u}-k_{n}\right)_{+}\right|^{p\left(1+\frac{2}{N}\right)} \zeta_{n}^{p\left(1+\frac{2}{N}\right)} \mathrm{d} x \mathrm{~d} t \geq\left(k_{n+1}-k_{n}\right)^{p \frac{N+2}{N}} A_{n+1} \geq\left(\frac{1}{2^{\bar{m}+n+2}}\right)^{p \frac{N+2}{N}} A_{n+1} .
$$

Combining this estimate with (6.48), we obtain

$$
Y_{n+1} \leq C_{4} 4^{p n\left(1+\frac{2}{N}\right)} Y_{n}^{1+\frac{p}{N}},
$$

for a constant $C_{4}$ depending only upon the data. At this point, we set

$$
v_{4}=4^{-\frac{N(N+2)}{p}} C_{4}^{-\frac{N}{p}} .
$$

Choose $v=v_{4}$, and hence $\bar{m}=\bar{m}\left(v_{4}\right)$, from this and (6.46). From Lemma 6.8, $Y_{0} \leq v_{4}$. By the lemma on fast geometric convergence of sequences, we infer that $Y_{n} \rightarrow 0$ as $n \rightarrow \infty$. This proves the lemma.

Transforming back to the original function $u$ and original variables $(x, t)$, we obtain the following DeGiorgi-type result.

Lemma 6.10 Let $\bar{m}>1$ be the constant as in Lemma 6.9. Then there exists a time level $t_{\omega}^{(0)} \in\left(\tilde{t}, t^{(0)}\right)$ such that

$$
u(x, t)<\mu_{+}-\frac{\omega}{2^{m_{2} p+\bar{m}+1}} \text { a.e. in }\left(0, t^{(0)}\right)+Q\left(\frac{t^{(0)}-t_{\omega}^{(0)}}{2^{p}}, 10 R^{(0)}\right),
$$

where $t_{\omega}^{(0)}=t^{(0)}+\left(t^{(0)}-\tilde{t}\right) t_{\omega}^{\prime}$.

To obtain the expansion of positivity at a higher time level $t^{(1)}$, we need the following lemma that is similar to Lemma 6.6.

Lemma 6.11 For any $v \in(0,1)$, there exist a constant $\delta_{1}$, depending only upon the data and $p$, and a time level

$$
t^{(1)}=t^{(0)}+\delta_{1} v \omega^{(1-p)(2-p)} R^{p}
$$

such that

$$
\left|\left\{x \in K_{6 R^{(0)}}: u(x, t)>\mu_{+}-\frac{\omega}{2^{m_{2} p+\bar{m}+4}}\right\}\right|<v\left|K_{6 R^{(0)}}\right|
$$

for any $t \in\left(t^{(0)}, t^{(1)}\right)$, and the constant $\delta_{1}$ is stable as $p \rightarrow 2$. 
Proof Let $k=\mu_{+}-\frac{\omega}{2^{m_{2} p+\bar{m}+1}}$ and $c=\frac{\omega}{2^{m_{2} p+\bar{m}+4}}$. We consider the logarithmic function

$$
\psi^{+}=\ln ^{+}\left(\frac{\frac{\omega}{2^{m_{2} p+\bar{m}+1}}}{\frac{\omega}{2^{m} 2^{p+\bar{m}+1}}-(u-k)_{+}+c}\right) .
$$

Then, we obtain $\psi^{+} \leq 8 \ln 2$ and

$$
\left[\left(\psi^{+}\right)^{\prime}\right]^{2-p} \leq\left(\frac{1}{c}\right)^{2-p} \leq\left(\frac{\omega}{2^{m_{2} p}}\right)^{p-2} 2^{(\bar{m}+4)(2-p)} .
$$

Choose a piecewise smooth cutoff function $\zeta(x)$, defined in $K_{10 R^{(0)}}$ and satisfying $0 \leq$ $\zeta \leq 1$ in $K_{10 R^{(0)}}, \zeta \equiv 1$ in $K_{6 R^{(0)}}$ and $|D \zeta| \leq\left(4 R^{(0)}\right)^{-1}$. Such cutoff functions can be chosen explicitly via the formulas (2.1)-(2.2). Since $k>\mu_{+}-\frac{\omega}{4}$, we check at once that $U\left(K_{10 R^{(0)}}, t^{(0)}, t^{(1)}, 2 \psi^{+}\left(\psi^{+}\right)^{\prime} \zeta^{p}\right)=0$. Then, we obtain from (3.4) the logarithmic estimate

$$
\begin{aligned}
& \underset{t^{(0)}<t<t^{(1)}}{\operatorname{ess} \sup _{K_{10 R^{(0)} \times\{t\}}}}\left(\psi^{+}\right)^{2} \zeta^{p} \mathrm{~d} x \leq \int_{K_{10 R^{(0)} \times\left\{t^{(0)}\right\}}}\left(\psi^{+}\right)^{2} \zeta^{p} \mathrm{~d} x \\
& +\gamma_{2} \int_{t^{(0)}}^{t^{(1)}} \int_{K_{10 R^{(0)}}} \psi^{+}\left|\left(\psi^{+}\right)^{\prime}\right|^{2-p}|D \zeta|^{p} \mathrm{~d} x \mathrm{~d} t .
\end{aligned}
$$

By Lemma 6.10, we see that $\int_{K_{10 R}(0)} \times\left\{t^{(0)}\right\}\left(\psi^{+}\right)^{2} \zeta^{p} \mathrm{~d} x=0$ and therefore,

$$
\begin{aligned}
& \underset{t^{(0)}<t<t^{(1)}}{\operatorname{ess} \sup _{K_{10 R^{(0)} \times\{t\}}}}\left(\psi^{+}\right)^{2} \zeta^{p} \mathrm{~d} x \\
& \leq 8 \gamma_{2}\left(\frac{\omega}{2^{m_{2} p}}\right)^{p-2} 2^{(\bar{m}+4)(2-p)}\left(4 R^{(0)}\right)^{-p}\left(t^{(1)}-t^{(0)}\right)(\ln 2)\left|K_{10 R^{(0)}}\right| \\
& =8 \frac{10^{N} \ln 2}{6^{N}} L_{2}^{-p} \gamma_{2} \omega^{(1-p)(p-2)} 2^{(\bar{m}+4)(2-p)} R^{-p}\left(t^{(1)}-t^{(0)}\right)\left|K_{6 R^{(0)}}\right| .
\end{aligned}
$$

At this stage, we choose the time level

$$
t^{(1)}=t^{(0)}+\delta_{1} v \omega^{(1-p)(2-p)} R^{p} \quad \text { where } \quad \delta_{1}=\frac{1}{8} \frac{6^{N} L_{2}^{p}}{10^{N} \gamma_{2} 2^{(\bar{m}+4)(2-p)} \ln 2} .
$$

Combining the estimates above, we infer that

$$
\underset{t^{(0)}<t<t^{(1)}}{\operatorname{ess} \sup _{K_{10 R^{(0)} \times\{t\}}}} \int_{\left.\psi^{+}\right)^{2} \zeta^{p} \mathrm{~d} x \leq \nu\left|K_{6 R^{(0)}}\right| .}
$$

On the other hand, we introduce the smaller set

$$
S=\left\{x \in K_{6 R^{(0)}}: u(x, t)>\mu_{+}-\frac{\omega}{2^{m_{2} p+\bar{m}+4}}\right\} \subset K_{6 R^{(0)}} .
$$

In this set, $\zeta \equiv 1$ and $\psi^{+} \geq 2 \ln 2>1$. Then, we conclude that

$$
\int_{K_{10 R^{(0)} \times\{t\}}}\left(\psi^{+}\right)^{2} \zeta^{p} \mathrm{~d} x \geq \int_{S \times\{t\}}\left(\psi^{+}\right)^{2} \zeta^{p} \mathrm{~d} x \geq\left|\left\{x \in K_{6 R^{(0)}}: u(x, t)>\mu_{+}-\frac{\omega}{2^{m_{2} p+\bar{m}+4}}\right\}\right|
$$

for all $t \in\left(t^{(0)}, t^{(1)}\right)$ and the lemma follows with the choice of $\delta_{1}$ in (6.53).

With the help of Lemma 6.11, we establish a DeGiorgi-type lemma and determine the value of $v$ and the time level $t^{(1)}$ in (6.51). This result extends (6.36) to a larger time interval. 
Lemma 6.12 Let $\delta_{1}>0$ be the constant chosen according to (6.53). Then, there exists a constant $v_{5}$ depending only upon the data and $p$ such that the following holds. For the choice

$$
t^{(1)}=t^{(0)}+\delta_{1} v_{5} \omega^{(1-p)(2-p)} R^{p},
$$

there holds

$$
u(x, t)<\mu_{+}-\frac{\omega}{2^{m_{2} p+\bar{m}+5}} \text { a.e. in } K_{2 R^{(0)}} \times\left(t^{(0)}, t^{(1)}\right]
$$

and the constant $\nu_{5}$ is stable as $p \rightarrow 2$.

Proof For $v \in(0,1)$ to be determined later, we take $\bar{m}=\bar{m}(v)>1$ according to (6.46). For $n=0,1,2, \ldots$, set

$$
R_{n}=2 R^{(0)}+\frac{4 R^{(0)}}{2^{n}}, \quad k_{n}=\mu_{+}-\frac{\omega}{2^{m_{2} p+\bar{m}+5}}-\frac{\omega}{2^{m_{2} p+\bar{m}+5+n}} \quad \text { and } \quad Q_{n}=K_{R_{n}} \times\left(t^{(0)}, t^{(1)}\right] .
$$

Let $\zeta_{n}=\zeta_{n}(x)$ be defined via (2.1)-(2.2) with $r$ and $r^{\prime}$ replaced by $R_{n+1}$ and $R_{n}$. Then, the cutoff function $\zeta_{n}$ satisfies $0 \leq \zeta_{n} \leq 1$ in $K_{R_{n}}, \zeta_{n} \equiv 1$ in $K_{R_{n+1}}$ and $\left|D \zeta_{n}\right| \leq 2^{n}\left(2 R^{(0)}\right)^{-1}$. Taking into account that $U\left(K_{R_{n}}, t^{(0)}, t^{(1)},\left(u-k_{n}\right)_{+} \zeta_{n}^{p}\right)=0$, we proceed similarly as in the proof of Proposition 6.7. Writing the energy estimates (3.3) for $\left(u-k_{n}\right)_{+}$over $Q_{n}$, we obtain

$$
\begin{aligned}
& \underset{\substack{\text { ess sup } \\
t^{(0)}<t<t^{(1)}}}{ } \int_{K_{R_{n}} \times\{t\}}\left(u-k_{n}\right)_{+}^{2} \zeta_{n}^{p} \mathrm{~d} x+\iint_{Q_{n}}\left|D\left(u-k_{n}\right)_{+} \zeta_{n}\right|^{p} \mathrm{~d} x \mathrm{~d} t \\
& \quad \leq \gamma_{1} \iint_{Q_{n}}\left(u-k_{n}\right)_{+}^{p}\left|D \zeta_{n}\right|^{p} \mathrm{~d} x \mathrm{~d} t .
\end{aligned}
$$

Set $A_{n}=\iint_{Q_{n}} \chi_{\left[\left(u-k_{n}\right)_{+}>0\right]} \mathrm{d} x \mathrm{~d} t$. Applying the parabolic Sobolev inequality (2.7), we deduce

$$
\begin{aligned}
& \iint_{Q_{n}}\left|\left(u-k_{n}\right)_{+} \zeta_{n}\right|^{p \frac{N+2}{N}} \mathrm{~d} x \mathrm{~d} t \\
& \quad \leq \gamma \iint_{Q_{n}}\left|D\left(u-k_{n}\right)_{+} \zeta_{n}\right|^{p} \mathrm{~d} x \mathrm{~d} t\left(\operatorname{ess}_{t^{(0)}<t<t^{(1)}} \int_{K_{R_{n} \times\{t\}}}\left(u-k_{n}\right)_{+}^{2} \zeta_{n}^{p} \mathrm{~d} x\right)^{\frac{p}{N}} \\
& \quad \leq \gamma \gamma_{1}^{1+\frac{p}{N}} \frac{2^{n p\left(1+\frac{p}{N}\right)}}{L_{2}^{p\left(1+\frac{p}{N}\right)}}\left(\frac{\omega^{(3-p) p}}{2^{2 p m_{2}}}\right)^{1+\frac{p}{N}}\left(\frac{1}{2^{\bar{m}+3}}\right)^{p\left(1+\frac{p}{N}\right)} R^{-p\left(1+\frac{p}{N}\right)} A_{n}^{1+\frac{p}{N}} .
\end{aligned}
$$

The integral on the left-hand side is estimated below by

$$
\iint_{Q_{n}}\left|\left(u-k_{n}\right)_{+} \zeta_{n}\right|^{p \frac{N+2}{N}} \mathrm{~d} x \mathrm{~d} t \geq\left(k_{n+1}-k_{n}\right)^{p \frac{N+2}{N}} A_{n+1}=\left(\frac{\omega}{2^{m_{2} p+\bar{m}+n+6}}\right)^{p \frac{N+2}{N}} A_{n+1} .
$$

Combining the estimates above, we see that

$$
\begin{aligned}
& A_{n+1} \leq \gamma \gamma_{1}^{1+\frac{p}{N}} 2^{n\left(2 p+\frac{2 p}{N}+\frac{p^{2}}{N}\right)} \omega^{-\frac{p^{3}}{N}+\left(\frac{3}{N}-1\right) p^{2}+2\left(1-\frac{1}{N}\right) p} 2^{m_{2} p(p-2)} R^{-p\left(1+\frac{p}{N}\right)} \\
& \frac{2^{p\left(1+\frac{p}{N}\right)} 4^{p \frac{N+2}{N}}}{L_{2}^{p\left(1+\frac{p}{N}\right)}}\left(\frac{1}{2^{\bar{m}+4}}\right)^{p\left(\frac{p-2}{N}\right)} A_{n}^{1+\frac{p}{N}} .
\end{aligned}
$$


Set $Y_{n}=A_{n} /\left|Q_{n}\right|$, and we will derive a recursive inequality. Noting that

$$
\begin{aligned}
\left|Q_{n}\right| & =\left(2 R^{(0)}+\frac{4 R^{(0)}}{2^{n}}\right)^{N}\left(t^{(1)}-t^{(0)}\right) \\
& =\left(2+2^{2-n}\right)^{N} \frac{1}{4^{N}}\left(\frac{\omega}{2^{m_{2}}}\right)^{(p-2) N} \delta_{1} \nu \omega^{(1-p)(2-p)} R^{N+p},
\end{aligned}
$$

we deduce

$$
\frac{1}{2^{N}}\left(\frac{\omega}{2^{m_{2}}}\right)^{(p-2) N} \delta_{1} v \omega^{(1-p)(2-p)} R^{N+p} \leq\left|Q_{n}\right| \leq \frac{3^{N}}{2^{N}}\left(\frac{\omega}{2^{m_{2}}}\right)^{(p-2) N} \delta_{1} v \omega^{(1-p)(2-p)} R^{N+p} .
$$

Moreover, this estimate implies

$$
\frac{\left|Q_{n+1}\right|}{\left|Q_{n}\right|} \geq \frac{1}{3^{N}}
$$

Since $0<v<1$, we conclude that

$$
\begin{aligned}
& Y_{n+1} \leq \frac{3^{N+p}}{2^{p}} \gamma \gamma_{1}^{1+\frac{p}{N}}\left(\delta_{1} v\right)^{\frac{p}{N}} 2^{n\left(2 p+\frac{2 p}{N}+\frac{p^{2}}{N}\right)} \\
& \frac{2^{p\left(1+\frac{p}{N}\right)} 4^{p \frac{N+2}{N}}}{L_{2}^{p\left(1+\frac{p}{N}\right)}}\left(\frac{1}{2^{\bar{m}+4}}\right)^{p\left(\frac{p-2}{N}\right)} Y_{n}^{1+\frac{p}{N}}=\delta_{2} b^{n} Y_{n}^{1+\frac{p}{N}},
\end{aligned}
$$

where

$$
b=2^{2 p+\frac{2 p}{N}+\frac{p^{2}}{N}} \quad \text { and } \quad \delta_{2}:=\frac{3^{p+N}}{2^{p}} \gamma \gamma_{1}^{1+\frac{p}{N}} \delta_{1}^{\frac{p}{N}} \frac{2^{p\left(1+\frac{p}{N}\right)} 4^{p \frac{N+2}{N}}}{L_{2}^{p\left(1+\frac{p}{N}\right)}}\left(\frac{1}{2^{\bar{m}+4}}\right)^{p\left(\frac{p-2}{N}\right)} .
$$

At this point, we set $\nu_{5}=\delta_{2}^{-\frac{N}{p}} b^{-\frac{N^{2}}{p^{2}}}$. Recalling the definition of $\delta_{1}$ from (6.53), we see that

$$
\delta_{2}=\frac{3^{p+N}}{2^{p}} \gamma \gamma_{1}^{1+\frac{p}{N}}\left(\frac{6^{N}}{8^{3-p} 10^{N} \gamma_{1} \ln 2}\right)^{\frac{p}{N}} \frac{2^{p\left(1+\frac{p}{N}\right)} 4^{p \frac{N+2}{N}}}{L_{2}^{p}}\left(\frac{1}{2^{3}}\right)^{p\left(\frac{p-2}{N}\right)},
$$

which is even independent of $\bar{m}$. Therefore, we conclude that if $Y_{0} \leq \delta_{2}^{-\frac{N}{p}} b^{-\frac{N^{2}}{p^{2}}}$ then $Y_{n} \rightarrow 0$ as $n \rightarrow \infty$. To this end, we choose $v=v_{5}$, and the lemma follows.

\subsection{Iterative arguments: time propagation of positivity from $t^{(1)}$ to $t^{(2)}$}

Starting from (6.54), we repeat the argument of Sect. 6.3 to obtain an estimate similar to (6.54) in a space-time cylinder containing a higher time level $t^{(2)}$. The argument is divided into four steps. To start with, we set $R^{(1)}=2 R^{(0)}$.

Step 1 We introduce the change of variables

$$
x^{\prime}=\frac{x}{20 R^{(1)}} \quad \text { and } \quad t^{\prime}=\frac{t-t^{(1)}}{t^{(1)}-t^{(0)}} .
$$

This transformation maps $K_{R^{(1)}} \times\left(t^{(0)}, t^{(1)}\right) \rightarrow K_{\frac{1}{20}} \times(-1,0)$ and $K_{20 R^{(1)}} \times\left(t^{(0)}, t^{(1)}\right) \rightarrow$ $Q_{1}$. We introduce the new functions

$$
\tilde{u}\left(x^{\prime}, t^{\prime}\right)=u(x, t) \text { and } \bar{u}\left(x^{\prime}, t^{\prime}\right)=\left(\tilde{u}\left(x^{\prime}, t^{\prime}\right)-\mu_{+}\right)\left(\frac{2^{m_{2} p+\bar{m}+5}}{\omega}\right) .
$$


From (6.54), we see that $\left|\left\{x^{\prime} \in K_{1}: \bar{u}\left(x^{\prime}, t^{\prime}\right)<-1\right\}\right| \geq 20^{-N}$ for all $t^{\prime} \in(-1,0)$. Set $\tilde{w}\left(x^{\prime}, t^{\prime}\right)=w(x, t)$, and $v(x, t)$ in (3.1) can be written in the new variables as

$$
\tilde{v}\left(x^{\prime}, t^{\prime}\right)= \begin{cases}\bar{v}, & \text { on }\left\{\bar{u}<-\mu_{+} \frac{2^{m_{2} p+\bar{m}+5}}{\omega}\right\}, \\ -\tilde{w}\left(x^{\prime}, t^{\prime}\right), & \text { on }\left\{\bar{u}=-\mu_{+} \frac{2^{m_{2} p+\bar{m}+5}}{\omega}\right\} .\end{cases}
$$

We rewrite the weak form (3.1) in terms of the new variables and new functions

$$
\begin{aligned}
-\frac{2^{m_{2} p+\bar{m}+5}}{\omega} \int_{K_{1}} \tilde{v}\left(\cdot, t^{\prime}\right) \chi\left\{\bar{u} \leq-\mu_{+} \frac{2^{m_{2} p+\bar{m}+5}}{\omega}\right\}^{\left.\varphi\left(\cdot, t^{\prime}\right) \mathrm{d} x^{\prime}\right|_{t^{\prime}=t_{1}} ^{t_{2}}} \\
+\frac{2^{m_{2} p+\bar{m}+5}}{\omega} \int_{t_{1}}^{t_{2}} \int_{K_{1}} \tilde{v} \chi\left\{\bar{u} \leq-\mu_{+} \frac{2^{m_{2} p+\bar{m}+5}}{\omega}\right\} \frac{\partial \varphi}{\partial t^{\prime}} \mathrm{d} x^{\prime} \mathrm{d} t^{\prime} \\
+\int_{t_{1}}^{t_{2}} \int_{K_{1}}\left(\varphi \frac{\partial \bar{u}}{\partial t^{\prime}}+\bar{A}\left(x^{\prime}, t^{\prime}, \bar{u}, D \bar{u}\right) \cdot D \varphi\right) \mathrm{d} x^{\prime} \mathrm{d} t^{\prime}=0
\end{aligned}
$$

for any $\varphi \in W_{p}\left(Q_{1}\right)$ and $\left[t_{1}, t_{2}\right] \subset(-1,0]$. It is easy to check that the vector field $\bar{A}$ satisfies the structure condition

$$
\left\{\begin{array}{l}
\bar{A}\left(x^{\prime}, t^{\prime}, \bar{u}, D \bar{u}\right) \cdot D \bar{u} \geq C_{0} \Lambda_{2}|D \bar{u}|^{p} \\
\left|\bar{A}\left(x^{\prime}, t^{\prime}, \bar{u}, D \bar{u}\right)\right| \leq C_{1} \Lambda_{2}|D \bar{u}|^{p-1}
\end{array}\right.
$$

where

$$
\Lambda_{2}=\left(\frac{\omega}{2^{m_{2} p+\bar{m}+5}}\right)^{p-2} \frac{t^{(1)}-t^{(0)}}{\left(20 R^{(1)}\right)^{p}} .
$$

According to (6.53)-(6.54), we deduce that

$$
\Lambda_{2}=\frac{2^{5(2-p)} \delta_{1} \nu_{5}}{10^{p} 2^{\bar{m}(p-2)} L_{2}^{p}}=\frac{2^{-1-p} 6^{N} \nu_{5}}{10^{N+p} \gamma_{2} \ln 2},
$$

which depends only upon the data and $p$ and is stable as $p \rightarrow 2$. For any $v \in(0,1)$, we proceed similarly as in the proof of Lemma 6.8 to conclude that there exists a constant $\gamma^{\prime \prime}$, depending only upon the data, such that the following holds. For the constant $m^{\prime}$ with the expression

$$
m^{\prime}=\exp \left\{\left(\frac{\gamma^{\prime \prime}}{v \Lambda_{2}}\right)^{2}\right\}
$$

there holds

$$
\left|\left\{x^{\prime} \in K_{\frac{3}{4}}: \bar{u}\left(x^{\prime}, t^{\prime}\right)>-2^{-m^{\prime}}\right\}\right| \leq v\left|K_{\frac{3}{4}}\right| \text { for all } t^{\prime} \in\left(-\left(\frac{3}{4}\right)^{p}, 0\right] .
$$

Step 2 Based on estimate (6.60), we are now in a position to obtain an upper bound for $u$ in a space-time cylinder with a larger cube in space. By the proofs of Lemmas 6.9 and 6.10, we conclude that there exists a time level $t_{\omega}^{(1)} \in\left(t^{(0)}, t^{(1)}\right)$ such that

$$
u(x, t)<\mu_{+}-\frac{\omega}{2^{m_{2} p+\bar{m}+5+m^{\prime}+1}} \quad \text { a.e. in }\left(0, t^{(1)}\right)+Q\left(\frac{t^{(1)}-t_{\omega}^{(1)}}{2^{p}}, 10 R^{(1)}\right)
$$

where

$$
m^{\prime}=\exp \left\{\left(\frac{\gamma^{\prime \prime}}{v_{4} \Lambda_{2}}\right)^{2}\right\}
$$


and the constant $\nu_{4}$ is chosen according to (6.49).

Step 3 Let $\delta_{1}>0$ be the constant chosen according to (6.53). For any $v \in(0,1)$, we claim that there exist a constant $M_{1}>0$, depending only upon the data and $p$, and a time level

$$
t^{(2)}=t^{(1)}+\delta_{1} v \omega^{(1-p)(2-p)} M_{1} R^{p}
$$

such that

$$
\left|\left\{x \in K_{6 R^{(1)}}: u(x, t)>\mu_{+}-\frac{\omega}{2^{m_{2} p+\bar{m}+5+m^{\prime}+4}}\right\}\right|<v\left|K_{6 R^{(1)}}\right|
$$

for any $t \in\left(t^{(1)}, t^{(2)}\right]$.

Proof of the claim Let $k=\mu_{+}-\frac{\omega}{2^{m_{2} p+\bar{m}+5+m^{\prime}+1}}$ and $c=\frac{\omega}{2^{m_{2} p+\bar{m}+5+m^{\prime}+4}}$. We consider the logarithmic function

$$
\psi^{+}=\ln ^{+}\left(\frac{\frac{\omega}{2^{m_{2} p+\bar{m}+5+m^{\prime}+1}}}{\frac{\omega}{2^{m_{2} p+\bar{m}+5+m^{\prime}+1}}-(u-k)_{+}+c}\right) .
$$

Then, we have $\psi^{+} \leq 8 \ln 2$ and

$$
\left[\left(\psi^{+}\right)^{\prime}\right]^{2-p} \leq\left(\frac{1}{c}\right)^{2-p} \leq\left(\frac{\omega}{2^{m_{2} p}}\right)^{p-2} 2^{\left(\bar{m}+5+m^{\prime}+4\right)(2-p)} .
$$

Let $\zeta=\zeta(x)$ be defined via (2.1)-(2.2) with $r$ and $r^{\prime}$ replaced by $6 R^{(1)}$ and $10 R^{(1)}$. It follows that $0 \leq \zeta \leq 1$ in $K_{10 R^{(1)}}, \zeta \equiv 1$ in $K_{6 R^{(1)}}$ and $|D \zeta| \leq\left(4 R^{(1)}\right)^{-1}$. From (6.61), we deduce $\int_{K_{10 R^{(1)}} \times\left\{t^{(1)}\right\}}\left(\psi^{+}\right)^{2} \zeta^{p} \mathrm{~d} x=0$. Taking into account that $U\left(K_{10 R^{(1)}}, t^{(1)}, t^{(2)}, 2 \psi^{+}\left(\psi^{+}\right)^{\prime} \zeta^{p}\right)=0$, we obtain from (3.4) the logarithmic estimate

$$
\operatorname{ess~sup}_{t^{(1)}<t<t^{(2)}} \int_{K_{10 R^{(1)} \times\{t\}}}\left(\psi^{+}\right)^{2} \zeta^{p} \mathrm{~d} x \leq \gamma_{2} \int_{t^{(1)}}^{t^{(2)}} \int_{K_{10 R^{(1)}}} \psi^{+}\left|\left(\psi^{+}\right)^{\prime}\right|^{2-p}|D \zeta|^{p} \mathrm{~d} x \mathrm{~d} t .
$$

Recalling the definition of $\delta_{1}$ from (6.53), we deduce

$$
\begin{aligned}
& \underset{t^{(1)}<t<t^{(2)}}{\operatorname{ess} \sup _{K_{10 R^{(1)} \times\{t\}}}}\left(\psi^{+}\right)^{2} \zeta^{p} \mathrm{~d} x \\
& \quad \leq 8 \gamma_{2}\left(\frac{\omega}{2^{m_{2} p}}\right)^{p-2} 2^{\left(\bar{m}+5+m^{\prime}+4\right)(2-p)}\left(4 R^{(1)}\right)^{-p}\left(t^{(2)}-t^{(1)}\right)(\ln 2)\left|K_{10 R^{(1)}}\right| \\
& \quad=v M_{1} 2^{\left(m^{\prime}+5\right)(2-p)} 2^{-p}\left|K_{6 R^{(1)}}\right| .
\end{aligned}
$$

At this stage, we set

$$
M_{1}=\frac{2^{p}}{2^{\left(m^{\prime}+5\right)(2-p)}} .
$$

It follows that

$$
\underset{t^{(1)}<t<t^{(2)}}{\operatorname{ess} \sup _{K_{10 R^{(1)}} \times\{t\}}}\left(\psi^{+}\right)^{2} \zeta^{p} \mathrm{~d} x \leq v\left|K_{6 R^{(1)}}\right| .
$$

On the other hand, we introduce the smaller set

$$
\left\{x \in K_{6 R^{(1)}}: u(x, t)>\mu_{+}-\frac{\omega}{2^{m_{2} p+\bar{m}+5+m^{\prime}+4}}\right\} \subset K_{6 R^{(1)}} .
$$


In this set, $\zeta \equiv 1$ and $\psi^{+} \geq 2 \ln 2>1$. Then, we conclude that

$$
\int_{K_{10 R^{(1)}} \times\{t\}}\left(\psi^{+}\right)^{2} \zeta^{p} \mathrm{~d} x \geq\left|\left\{x \in K_{6 R^{(1)}}: u(x, t)>\mu_{+}-\frac{\omega}{2^{m_{2} p+\bar{m}+5+m^{\prime}+4}}\right\}\right|
$$

for all $t \in\left(t^{(1)}, t^{(2)}\right)$, and the estimate (6.63) follows.

Step 4 Take $v_{5}=\delta_{2}^{-\frac{N}{p}} b^{-\frac{N^{2}}{p^{2}}}$ be the constant as in Lemma 6.12. We claim that for the choice

$$
t^{(2)}=t^{(1)}+\delta_{1} v_{5} \omega^{(1-p)(2-p)} M_{1} R^{p},
$$

there holds

$$
u(x, t)<\mu_{+}-\frac{\omega}{2^{m_{2} p+\bar{m}+5+m^{\prime}+5}} \quad \text { a.e. in } K_{2 R^{(1)}} \times\left(t^{(1)}, t^{(2)}\right] .
$$

Proof of the claim Proceed similarly as in the proof of Lemma 6.12, we consider two decreasing sequences of real numbers

$R_{n}=2 R^{(1)}+\frac{4 R^{(1)}}{2^{n}}$ and $k_{n}=\mu_{+}-\frac{\omega}{2^{m_{2} p+\bar{m}+5+m^{\prime}+5}}-\frac{\omega}{2^{m_{2} p+\bar{m}+5+m^{\prime}+5+n}} \quad n=0,1,2, \ldots$

and set $Q_{n}=K_{R_{n}} \times\left(t^{(1)}, t^{(2)}\right]$. Let $\zeta_{n}=\zeta_{n}(x)$ be defined via (2.1)-(2.2) with $r$ and $r^{\prime}$ replaced by $R_{n+1}$ and $R_{n}$. Then, the cutoff function $\zeta_{n}$ satisfies $0 \leq \zeta_{n} \leq 1$ in $K_{R_{n}}, \zeta_{n} \equiv 1$ in $K_{R_{n+1}}$ and $\left|D \zeta_{n}\right| \leq 2^{n}\left(2 R^{(1)}\right)^{-1}$. Taking into account that $U\left(K_{R_{n}}, t^{(1)}, t^{(2)},\left(u-k_{n}\right)_{+} \zeta_{n}^{p}\right)=$ 0 , we write the energy estimate (3.3) for $\left(u-k_{n}\right)_{+}$over the cylinder $Q_{n}$ and obtain

$$
\begin{aligned}
\underset{t^{(1)}<t<t^{(2)}}{\operatorname{ess~sup}} \int_{K_{R_{n}} \times\{t\}} & \left(u-k_{n}\right)_{+}^{2} \zeta_{n}^{p} \mathrm{~d} x+\iint_{Q_{n}}\left|D\left(u-k_{n}\right)_{+} \zeta_{n}\right|^{p} \mathrm{~d} x \mathrm{~d} t \\
\leq & \gamma_{1}\left(\frac{\omega}{2^{m_{2} p+\bar{m}+5+m^{\prime}+4}}\right)^{p} \frac{2^{n p}}{\left(2 R^{(1)}\right)^{p}} A_{n} \\
& =\gamma_{1}\left(\frac{\omega}{2^{m_{2} p+\bar{m}+4}}\right)^{p} \frac{2^{n p}}{\left(2 R^{(0)}\right)^{p}} A_{n}\left(\frac{2^{-5 p-m^{\prime} p}}{2^{p}}\right),
\end{aligned}
$$

where $A_{n}=\iint_{Q_{n}} \chi_{\left[\left(u-k_{n}\right)_{+}>0\right]} \mathrm{d} x \mathrm{~d} t$. Applying the parabolic Sobolev inequality (2.7), we have

$$
\begin{aligned}
& \iint_{Q_{n}}\left|\left(u-k_{n}\right)_{+} \zeta_{n}\right|^{p \frac{N+2}{N}} \mathrm{~d} x \mathrm{~d} t \leq \gamma \iint_{Q_{n}}\left|D\left(u-k_{n}\right)_{+} \zeta_{n}\right|^{p} \mathrm{~d} x \mathrm{~d} t \\
& \left({\operatorname{ess~} \sup _{t^{(1)}<t<t^{(2)}}}_{K_{R_{n} \times\{t\}}}\left(u-k_{n}\right)_{+}^{2} \zeta_{n}^{p} \mathrm{~d} x\right)^{\frac{p}{N}} \\
& \leq \gamma \gamma_{1}^{1+\frac{p}{N}} \frac{2^{n p\left(1+\frac{p}{N}\right)}}{L_{2}^{p\left(1+\frac{p}{N}\right)}} \\
& \left(\frac{\omega^{(3-p) p}}{2^{2 p m_{2}}}\right)^{1+\frac{p}{N}} R^{-p\left(1+\frac{p}{N}\right)}\left(\frac{1}{2^{\bar{m}+3}}\right)^{p\left(1+\frac{p}{N}\right)} A_{n}^{1+\frac{p}{N}}\left(\frac{2^{-5 p-m^{\prime} p}}{2^{p}}\right)^{1+\frac{p}{N}} .
\end{aligned}
$$

To estimate below for the left-hand side, observe that

$$
k_{n+1}-k_{n}=\frac{\omega}{2^{m_{2} p+\bar{m}+5+m^{\prime}+n+6}} .
$$


This yields

$$
\begin{gathered}
\iint_{Q_{n}}\left|\left(u-k_{n}\right)_{+} \zeta_{n}\right|^{p \frac{N+2}{N}} \mathrm{~d} x \mathrm{~d} t \geq\left(k_{n+1}-k_{n}\right)^{p \frac{N+2}{N}} A_{n+1} \\
=\left(\frac{\omega}{2^{m_{2} p+\bar{m}+n+6}}\right)^{p \frac{N+2}{N}} A_{n+1}\left(\frac{1}{2^{m^{\prime}+5}}\right)^{p \frac{N+2}{N}} .
\end{gathered}
$$

Consequently, we infer that

$$
\begin{aligned}
A_{n+1} \leq & \gamma \gamma_{1}^{1+\frac{p}{N}} 2^{n\left(2 p+\frac{2 p}{N}+\frac{p^{2}}{N}\right)} \omega^{-\frac{p^{3}}{N}+\left(\frac{3}{N}-1\right) p^{2}+2\left(1-\frac{1}{N}\right) p} 2^{m_{2} p(p-2)} R^{-p\left(1+\frac{p}{N}\right)} \\
& \frac{2^{p\left(1+\frac{p}{N}\right)} 4^{p \frac{N+2}{N}}}{L_{2}^{p\left(1+\frac{p}{N}\right)}}\left(\frac{1}{2^{\bar{m}+4}}\right)^{p\left(\frac{p-2}{N}\right)} \\
& \times A_{n}^{1+\frac{p}{N}} 2^{\left(m^{\prime}+5\right) p \frac{2-p}{N}} 2^{-p\left(1+\frac{p}{N}\right)} .
\end{aligned}
$$

Taking into account that

$$
\begin{aligned}
\left|Q_{n}\right| & =\left(2 R^{(1)}+\frac{4 R^{(1)}}{2^{n}}\right)^{N}\left(t^{(2)}-t^{(1)}\right) \\
& =\left(2+2^{2-n}\right)^{N} \frac{1}{4^{N}}\left(\frac{\omega}{2^{m_{2}}}\right)^{(p-2) N} \delta_{1} v_{5} \omega^{(1-p)(2-p)} R^{N+p}\left(2^{N} M_{1}\right),
\end{aligned}
$$

then there holds

$$
\begin{gathered}
\left(2^{N} M_{1}\right) \frac{1}{2^{N}}\left(\frac{\omega}{2^{m_{2}}}\right)^{(p-2) N} \delta_{1} v_{5} \omega^{(1-p)(2-p)} R^{N+p} \leq\left|Q_{n}\right| \\
\leq\left(2^{N} M_{1}\right) \frac{3^{N}}{2^{N}}\left(\frac{\omega}{2^{m_{2}}}\right)^{(p-2) N} \delta_{1} v_{5} \omega^{(1-p)(2-p)} R^{N+p}
\end{gathered}
$$

and

$$
\frac{\left|Q_{n+1}\right|}{\left|Q_{n}\right|} \geq \frac{1}{3^{N}}
$$

Set $Y_{n}=A_{n} /\left|Q_{n}\right|$. Recalling the definition of $M_{1}$ and noting that $0<v_{5}<1$, we deduce

$$
\begin{aligned}
Y_{n+1} \leq & \frac{3^{N+p}}{2^{p}} \gamma \gamma_{1}^{1+\frac{p}{N}}\left(\delta_{1} v_{5}\right)^{\frac{p}{N}} 2^{n\left(2 p+\frac{2 p}{N}+\frac{p^{2}}{N}\right)} \\
& \frac{2^{p\left(1+\frac{p}{N}\right)} 4^{p \frac{N+2}{N}}}{L_{2}^{p\left(1+\frac{p}{N}\right)}}\left(\frac{1}{2^{\bar{m}+4}}\right)^{p\left(\frac{p-2}{N}\right)} Y_{n}^{1+\frac{p}{N}} 2^{\left(m^{\prime}+5\right) p \frac{2-p}{N}} 2^{-p\left(1+\frac{p}{N}\right)}\left(2^{N} M_{1}\right)^{\frac{p}{N}} \\
\leq & \delta_{2} b^{n} Y_{n}^{1+\frac{p}{N}}
\end{aligned}
$$

since

$$
2^{\left(m^{\prime}+5\right) p \frac{2-p}{N}} 2^{-p\left(1+\frac{p}{N}\right)}\left(2^{N} M_{1}\right)^{\frac{p}{N}}=2^{\left(m^{\prime}+5\right) p \frac{2-p}{N}} 2^{-p\left(1+\frac{p}{N}\right)} 2^{p}\left(\frac{2^{p}}{2^{\left(m^{\prime}-5\right)(2-p)}}\right)^{\frac{p}{N}}=1 .
$$

Then, we conclude that if $Y_{0} \leq v_{5}=\delta_{2}^{-\frac{N}{p}} b^{-\frac{N^{2}}{p^{2}}}$ then $Y_{n} \rightarrow 0$ as $n \rightarrow \infty$. To this end, we choose $v=v_{5}$ in (6.63), and the claim follows. 


\subsection{Iterative arguments: time propagation of positivity from $t^{(k)}$ to $t^{(k+1)}(k \geq 2)$}

Starting from (6.66), we can repeat the argument of Sect. 6.4 at any time to obtain a sequence of time levels $\left\{t^{(k)}\right\}_{k=1}^{\infty}$. Henceforth, set

$$
\begin{aligned}
R^{(k)} & =2^{k} R^{(0)}, \quad s_{k}=m_{2} p+\bar{m}+5+\left(m^{\prime}+5\right)(k-1) \quad \text { and } \\
t^{(k)} & =t^{(k-1)}+\delta_{1} v_{5} \omega^{(1-p)(2-p)} M_{1}^{k-1} R^{p} .
\end{aligned}
$$

We now claim that

$$
u(x, t)<\mu_{+}-\frac{\omega}{2^{s_{k}}} \text { a.e. in } K_{R^{(k)}} \times\left(t^{(k-1)}, t^{(k)}\right]
$$

for any $k \geq 2$.

Proof of (6.68) We first observe from (6.66) that (6.68) holds with $k=2$. Assuming (6.68) to hold for $k$, we will prove it for $k+1$. We proceed similarly as in Sect. 6.4 and divide the proof into four steps.

Step 1 Let $\left(x^{\prime}, t^{\prime}\right)$ be the new variables defined by

$$
x^{\prime}=\frac{x}{20 R^{(k)}} \text { and } \quad t^{\prime}=\frac{t-t^{(k)}}{t^{(k)}-t^{(k-1)}} .
$$

This transformation maps $K_{R^{(k)}} \times\left(t^{(k-1)}, t^{(k)}\right) \rightarrow K_{\frac{1}{20}} \times(-1,0)$ and $K_{20 R^{(k)}} \times$ $\left(t^{(k-1)}, t^{(k)}\right) \rightarrow Q_{1}$. Set

$$
\tilde{u}\left(x^{\prime}, t^{\prime}\right)=u(x, t) \text { and } \bar{u}\left(x^{\prime}, t^{\prime}\right)=\left(\tilde{u}\left(x^{\prime}, t^{\prime}\right)-\mu_{+}\right)\left(\frac{2^{s_{k}}}{\omega}\right) .
$$

By the induction hypothesis, we note that $\left|\left\{x^{\prime} \in K_{1}: \bar{u}\left(x^{\prime}, t^{\prime}\right)<-1\right\}\right| \geq 20^{-N}$ for all $t^{\prime} \in(-1,0)$. Set $\tilde{w}\left(x^{\prime}, t^{\prime}\right)=w(x, t)$, and $v(x, t)$ in (3.1) can be written in the new variables as

$$
\tilde{v}\left(x^{\prime}, t^{\prime}\right)= \begin{cases}\bar{v}, & \text { on }\left\{\bar{u}<-\mu_{+} \frac{2^{s_{k}}}{\omega}\right\}, \\ -\tilde{w}\left(x^{\prime}, t^{\prime}\right), & \text { on }\left\{\bar{u}=-\mu_{+} \frac{2^{s_{k}}}{\omega}\right\} .\end{cases}
$$

We rewrite the weak form (3.1) in terms of the new variables and new functions

$$
\begin{gathered}
-\left.\frac{2^{s_{k}}}{\omega} \int_{K_{1}} \tilde{v}\left(\cdot, t^{\prime}\right) \chi_{\left\{\bar{u} \leq-\mu_{+} \frac{2^{s_{k}}}{\omega}\right\}} \varphi\left(\cdot, t^{\prime}\right) \mathrm{d} x^{\prime}\right|_{t^{\prime}=t_{1}} ^{t_{2}}+\frac{2^{s_{k}}}{\omega} \int_{t_{1}}^{t_{2}} \int_{K_{1}} \tilde{v} \chi_{\left\{\bar{u} \leq-\mu_{+} \frac{2^{s_{k}}}{\omega}\right\}} \frac{\partial \varphi}{\partial t^{\prime}} \mathrm{d} x^{\prime} \mathrm{d} t^{\prime} \\
+\int_{t_{1}}^{t_{2}} \int_{K_{1}}\left(\varphi \frac{\partial \bar{u}}{\partial t^{\prime}}+\bar{A}\left(x^{\prime}, t^{\prime}, \bar{u}, D \bar{u}\right) \cdot D \varphi\right) \mathrm{d} x^{\prime} \mathrm{d} t^{\prime}=0
\end{gathered}
$$

for any $\varphi \in W_{p}\left(Q_{1}\right)$ and $\left[t_{1}, t_{2}\right] \subset(-1,0]$. We check at once that $\bar{A}$ satisfies the structure condition

$$
\left\{\begin{array}{l}
\bar{A}\left(x^{\prime}, t^{\prime}, \bar{u}, D \bar{u}\right) \cdot D \bar{u} \geq C_{0} \Lambda_{k}|D \bar{u}|^{p}, \\
\left|\bar{A}\left(x^{\prime}, t^{\prime}, \bar{u}, D \bar{u}\right)\right| \leq C_{1} \Lambda_{k}|D \bar{u}|^{p-1}
\end{array}\right.
$$

where

$$
\Lambda_{k}=\left(\frac{\omega}{2^{s_{k}}}\right)^{p-2} \frac{t^{(k)}-t^{(k-1)}}{\left(20 R^{(k)}\right)^{p}} .
$$


Taking into account the definitions of $s_{k}, R^{(k)}, t^{(k)}, M_{1}$ and $\Lambda_{2}$, we infer that

$$
\begin{aligned}
\Lambda_{k} & =\left(\frac{\omega}{2^{m_{2} p+\bar{m}+5+\left(m^{\prime}+5\right)(k-1)}}\right)^{p-2} \frac{\delta_{1} v_{5} \omega^{(1-p)(2-p)} M_{1}^{k-1} R^{p}}{20^{p} 2^{k p} 4^{-p} L_{2}^{p}\left(\frac{\omega}{\left.2^{m}\right)^{(p-2) p}} R^{p}\right.} \\
& =\left(\frac{1}{2^{\bar{m}+5+\left(m^{\prime}+5\right)(k-1)}}\right)^{p-2} \frac{\delta_{1} v_{5}}{20^{p} 2^{k p} 4^{-p} L_{2}^{p}}\left(\frac{2^{p}}{2^{\left(m^{\prime}+5\right)(2-p)}}\right)^{k-1} \\
& =\frac{2^{5(2-p)} \delta_{1} v_{5}}{10^{p} 2^{\bar{m}(p-2)} L_{2}^{p}}=\Lambda_{2} .
\end{aligned}
$$

At this point, the proof now is exactly the same as in step 1 in Sect. 6.4. For any $v \in(0,1)$, we choose $m^{\prime}$ as in (6.59) and conclude that

$$
\left|\left\{x^{\prime} \in K_{\frac{3}{4}}: \bar{u}\left(x^{\prime}, t^{\prime}\right)>-2^{-m^{\prime}}\right\}\right| \leq v\left|K_{\frac{3}{4}}\right| \text { for all } t^{\prime} \in\left(-\left(\frac{3}{4}\right)^{p}, 0\right] .
$$

Step 2 With the previous result in hand, we proceed similarly as in step 1 in Sect. 6.4 to conclude that there exists a time level $t_{\omega}^{(k)} \in\left(t^{(k-1)}, t^{(k)}\right)$ such that

$$
u(x, t)<\mu_{+}-\frac{\omega}{2^{s_{k}+m^{\prime}+1}} \quad \text { a.e. in }\left(0, t^{(k)}\right)+Q\left(\frac{t^{(k)}-t_{\omega}^{(k)}}{2^{p}}, 10 R^{(k)}\right),
$$

where $m^{\prime}$ is the same constant as in (6.62).

Step 3 For any $v \in(0,1)$, we claim that there exists a time level

$$
t^{(k+1)}=t^{(k)}+\delta_{1} v \omega^{(1-p)(2-p)} M_{1}^{k} R^{p}
$$

such that

$$
\left|\left\{x \in K_{6 R^{(k)}}: u(x, t)>\mu_{+}-\frac{\omega}{2^{s_{k}+m^{\prime}+4}}\right\}\right|<v\left|K_{6 R^{(k)}}\right|
$$

for any $t \in\left(t^{(k)}, t^{(k+1)}\right]$.

Proof of the claim Let $k^{\prime}=\mu_{+}-\frac{\omega}{2^{s_{k}+m^{\prime}+1}}$ and $c=\frac{\omega}{2^{s_{k}+m^{\prime}+4}}$. We consider the logarithmic function

$$
\psi^{+}=\ln ^{+}\left(\frac{\frac{\omega}{2^{s_{k}+m^{\prime}+1}}}{\frac{\omega}{2^{s_{k}+m^{\prime}+1}}-\left(u-k^{\prime}\right)_{+}+c}\right) .
$$

Taking into account the definition of $s_{k}$, we have $\psi^{+} \leq 8 \ln 2$ and

$$
\left[\left(\psi^{+}\right)^{\prime}\right]^{2-p} \leq\left(\frac{1}{c}\right)^{2-p} \leq\left(\frac{\omega}{2^{m_{2} p}}\right)^{p-2} 2^{\left(\bar{m}+5+\left(m^{\prime}+5\right)(k-1)+m^{\prime}+4\right)(2-p)} .
$$

Let $\zeta=\zeta(x)$ be defined via (2.1)-(2.2) with $r$ and $r^{\prime}$ replaced by $6 R^{(k)}$ and $10 R^{(k)}$. It is clear that $0 \leq \zeta \leq 1$ in $K_{10 R^{(k)}}, \zeta \equiv 1$ in $K_{6 R^{(k)}}$ and $|D \zeta| \leq\left(4 R^{(k)}\right)^{-1}$. It follows from (6.72) that $\int_{K_{10 R^{(k)}} \times\left\{t^{(k)}\right\}}\left(\psi^{+}\right)^{2} \zeta^{p} \mathrm{~d} x=0$. Taking into account that $U\left(K_{10 R^{(k)}}, t^{(k)}, t^{(k+1)}, 2 \psi^{+}\left(\psi^{+}\right)^{\prime} \zeta^{p}\right)=0$, we obtain from (3.4) the logarithmic estimate

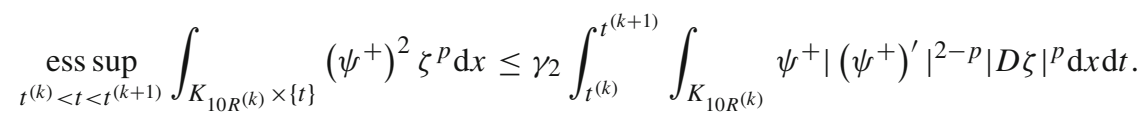


Recalling the definitions of $\delta_{1}$ and $M_{1}$, we obtain

$$
\begin{aligned}
\underset{t^{(k)}<t<t^{(k+1)}}{\operatorname{ess} \sup } & \int_{K_{10 R^{(k)} \times\{t\}}}\left(\psi^{+}\right)^{2} \zeta^{p} \mathrm{~d} x \\
& \leq 8 \gamma_{2}\left(\frac{\omega}{2^{m_{2} p}}\right)^{p-2} 2^{(\bar{m}+4)(2-p)}\left(4 R^{(0)}\right)^{-p}\left(t^{(k+1)}-t^{(k)}\right)(\ln 2)\left|K_{10 R^{(k)}}\right|\left(\frac{2^{\left(5+m^{\prime}\right)(2-p) k}}{2^{k p}}\right) \\
& =v M_{1}^{k}\left(\frac{2^{\left(5+m^{\prime}\right)(2-p) k}}{2^{k p}}\right)\left|K_{6 R^{(k)}}\right|=v\left|K_{6 R^{(k)}}\right| .
\end{aligned}
$$

On the other hand, we introduce the smaller set

$$
\left\{x \in K_{6 R^{(k)}}: u(x, t)>\mu_{+}-\frac{\omega}{2^{s_{k}+m^{\prime}+4}}\right\} \subset K_{6 R^{(k)}} .
$$

In this set, $\zeta \equiv 1$ and $\psi^{+} \geq 2 \ln 2>1$. Then, we conclude that

$$
\int_{K_{10 R^{(1)}} \times\{t\}}\left(\psi^{+}\right)^{2} \zeta^{p} \mathrm{~d} x \geq\left|\left\{x \in K_{6 R^{(1)}}: u(x, t)>\mu_{+}-\frac{\omega}{2^{s_{k}+m^{\prime}+4}}\right\}\right|
$$

for all $t \in\left(t^{(k)}, t^{(k+1)}\right)$ and the estimate (6.73) follows.

Step 4 Proof of estimate (6.68) for $k+1$. For $n=0,1,2, \ldots$, set

$$
R_{n}=2 R^{(k)}+\frac{4 R^{(k)}}{2^{n}}, \quad k_{n}=\mu_{+}-\frac{\omega}{2^{s_{k}+m^{\prime}+5}}-\frac{\omega}{2^{s_{k}+m^{\prime}+5+n}} \quad \text { and } \quad Q_{n}=K_{R_{n}} \times\left(t^{(k)}, t^{(k+1)}\right] .
$$

Let $\zeta_{n}=\zeta_{n}(x)$ be defined via (2.1)-(2.2) with $r$ and $r^{\prime}$ replaced by $R_{n+1}$ and $R_{n}$. Then the cutoff function $\zeta_{n}$ satisfies $0 \leq \zeta_{n} \leq 1$ in $K_{R_{n}}, \zeta_{n} \equiv 1$ in $K_{R_{n+1}}$ and $\left|D \zeta_{n}\right| \leq 2^{n}\left(2 R^{(k)}\right)^{-1}$. Taking into account that $U\left(K_{R_{n}}, t^{(k)}, t^{(k+1)},\left(u-k_{n}\right)_{+} \zeta_{n}^{p}\right)=0$, we write the energy estimate (3.3) for $\left(u-k_{n}\right)_{+}$over the cylinder $Q_{n}$ and obtain

$$
\begin{aligned}
& \underset{t^{(k)}<t<t^{(k+1)}}{\operatorname{ess} \sup _{K_{R_{n}} \times\{t\}}}\left(u-k_{n}\right)_{+}^{2} \zeta_{n}^{p} \mathrm{~d} x+\iint_{Q_{n}}\left|D\left(u-k_{n}\right)_{+} \zeta_{n}\right|^{p} \mathrm{~d} x \mathrm{~d} t \\
& \leq \gamma_{1}\left(\frac{\omega}{2^{s_{k}+m^{\prime}+4}}\right)^{p} \frac{2^{n p}}{\left(2 R^{(k)}\right)^{p}} A_{n} \\
& =\gamma_{1}\left(\frac{\omega}{2^{m_{2} p+\bar{m}+4}}\right)^{p} \frac{2^{n p}}{\left(2 R^{(0)}\right)^{p}} A_{n}\left(\frac{2^{-\left(5+m^{\prime}\right) k p}}{2^{k p}}\right) \text {, }
\end{aligned}
$$

where $A_{n}=\iint_{Q_{n}} \chi_{\left[\left(u-k_{n}\right)_{+}>0\right]} \mathrm{d} x \mathrm{~d} t$. Applying the parabolic Sobolev inequality (2.7), we have

$$
\begin{aligned}
& \iint_{Q_{n}}\left|\left(u-k_{n}\right)_{+} \zeta_{n}\right|^{p \frac{N+2}{N}} \mathrm{~d} x \mathrm{~d} t \leq \gamma \iint_{Q_{n}}\left|D\left(u-k_{n}\right)_{+} \zeta_{n}\right|^{p} \mathrm{~d} x \mathrm{~d} t \\
& \left(\operatorname{ess}_{t^{(k)}<t<t^{(k+1)}} \int_{K_{R_{n}} \times\{t\}}\left(u-k_{n}\right)_{+}^{2} \zeta_{n}^{p} \mathrm{~d} x\right)^{\frac{p}{N}} \\
& \leq \gamma \gamma_{1}^{1+\frac{p}{N}} \frac{2^{n p\left(1+\frac{p}{N}\right)}}{L_{2}^{p\left(1+\frac{p}{N}\right)}}\left(\frac{\omega^{(3-p) p}}{2^{2 p m_{2}}}\right)^{1+\frac{p}{N}} \\
& R^{-p\left(1+\frac{p}{N}\right)}\left(\frac{1}{2^{\bar{m}+3}}\right)^{p\left(1+\frac{p}{N}\right)} A_{n}^{1+\frac{p}{N}}\left(\frac{2^{-\left(5+m^{\prime}\right) k p}}{2^{k p}}\right)^{1+\frac{p}{N}} .
\end{aligned}
$$


To estimate below for the left-hand side, take into account that

$$
k_{n+1}-k_{n}=\frac{\omega}{2^{s_{k}+m^{\prime}+n+6}}=\frac{\omega}{2^{m_{2} p+\bar{m}+n+6}} \frac{1}{2^{\left(m^{\prime}+5\right) k}} .
$$

This yields

$$
\begin{aligned}
& \iint_{Q_{n}}\left|\left(u-k_{n}\right)_{+} \zeta_{n}\right|^{p \frac{N+2}{N}} \mathrm{~d} x \mathrm{~d} t \geq\left(k_{n+1}-k_{n}\right)^{p \frac{N+2}{N}} A_{n+1} \\
& =\left(\frac{\omega}{2^{m_{2} p+\bar{m}+n+6}}\right)^{p \frac{N+2}{N}} A_{n+1}\left(\frac{1}{2^{\left(m^{\prime}+5\right) k}}\right)^{p \frac{N+2}{N}} .
\end{aligned}
$$

Combining the estimates above, we infer that

$$
\begin{aligned}
A_{n+1} \leq & \gamma \gamma_{1}^{1+\frac{p}{N}} 2^{n\left(2 p+\frac{2 p}{N}+\frac{p^{2}}{N}\right)} \omega^{-\frac{p^{3}}{N}+\left(\frac{3}{N}-1\right) p^{2}+2\left(1-\frac{1}{N}\right) p} 2^{m_{2} p(p-2)} \\
& R^{-p\left(1+\frac{p}{N}\right)} \frac{2^{p\left(1+\frac{p}{N}\right)} 4^{p \frac{N+2}{N}}}{L_{2}^{p\left(1+\frac{p}{N}\right)}}\left(\frac{1}{2^{\bar{m}+4}}\right)^{p\left(\frac{p-2}{N}\right)} \\
& \times A_{n}^{1+\frac{p}{N}} 2^{k\left(m^{\prime}+5\right) p \frac{2-p}{N}} 2^{-k p\left(1+\frac{p}{N}\right)} .
\end{aligned}
$$

Taking into account that

$$
\begin{aligned}
\left|Q_{n}\right| & =\left(2 R^{(k)}+\frac{4 R^{(k)}}{2^{n}}\right)^{N}\left(t^{(k+1)}-t^{(k)}\right) \\
& =\left(2+2^{2-n}\right)^{N} \frac{1}{4^{N}}\left(\frac{\omega}{2^{m_{2}}}\right)^{(p-2) N} \delta_{1} v_{5} \omega^{(1-p)(2-p)} R^{N+p}\left(2^{N} M_{1}\right)^{k},
\end{aligned}
$$

we arrive at

$$
\begin{aligned}
& \left(2^{N} M_{1}\right)^{k} \frac{1}{2^{N}}\left(\frac{\omega}{2^{m_{2}}}\right)^{(p-2) N} \delta_{1} v_{5} \omega^{(1-p)(2-p)} R^{N+p} \leq\left|Q_{n}\right| \leq\left(2^{N} M_{1}\right)^{k} \frac{3^{N}}{2^{N}} \\
& \left(\frac{\omega}{2^{m_{2}}}\right)^{(p-2) N} \delta_{1} v_{5} \omega^{(1-p)(2-p)} R^{N+p}
\end{aligned}
$$

and

$$
\frac{\left|Q_{n+1}\right|}{\left|Q_{n}\right|} \geq \frac{1}{3^{N}}
$$

Set $Y_{n}=A_{n} /\left|Q_{n}\right|$. Recalling the definition of $M_{1}$ and noting that $\nu_{5} \in(0,1)$, we deduce from (6.67) that

$$
\begin{aligned}
Y_{n+1} \leq & \frac{3^{N+p}}{2^{p}} \gamma \gamma_{1}^{1+\frac{p}{N}}\left(\delta_{1} v_{5}\right)^{\frac{p}{N}} 2^{n\left(2 p+\frac{2 p}{N}+\frac{p^{2}}{N}\right)} \frac{2^{p\left(1+\frac{p}{N}\right)} 4^{p \frac{N+2}{N}}}{L_{2}^{p\left(1+\frac{p}{N}\right)}}\left(\frac{1}{2^{\bar{m}+4}}\right)^{p\left(\frac{p-2}{N}\right)} Y_{n}^{1+\frac{p}{N}} \\
& \times\left(2^{\left(m^{\prime}+5\right) p \frac{2-p}{N}} 2^{-p\left(1+\frac{p}{N}\right)}\left(2^{N} M_{1}\right)^{\frac{p}{N}}\right)^{k} \\
\leq & \delta_{2} b^{n} Y_{n}^{1+\frac{p}{N}} .
\end{aligned}
$$

Therefore, we conclude that if $Y_{0} \leq v_{5}=\delta_{2}^{-\frac{N}{p}} b^{-\frac{N^{2}}{p^{2}}}$ then $Y_{n} \rightarrow 0$ as $n \rightarrow \infty$. To this end, we choose $v=v_{5}$ in (6.73), and estimate (6.68) follows for $k+1$. 


\subsection{Analysis of the second alternative concluded}

Now, we are prepared to proceed to obtain a DeGiorgi-type result similar to Proposition 5.4. Let us assume initially that the process in Sect. 6.5 can be iterated an unlimited number of times. We conclude from (6.36), (6.54) and (6.68) that for any $j \geq 0$, the following holds

$$
u(x, t)<\mu_{+}-\frac{\omega}{2^{s_{j}}} \text { a.e. in } K_{\frac{1}{4} c_{2} R} \times\left(\tilde{t}, t^{(j)}\right] .
$$

Note that the time level $t^{(j)}$ should be sufficiently large such that $\bar{t} \in\left(\tilde{t}, t^{(j)}\right]$. For this purpose, we have to impose the condition that $M_{1} \geq 1$. Since $m^{\prime}$ is uniformly bounded in $p$, we set $m^{\prime \prime}=\sup _{\frac{3}{2}<p \leq 2} m^{\prime}$ and impose a condition for $p$ as follows:

$$
\frac{2\left(m^{\prime \prime}+5\right)}{m^{\prime \prime}+6} \leq p<2
$$

In this case, we see that $t^{(j)} \rightarrow+\infty$ as $j \rightarrow+\infty$, and we can find an integer $j_{*}$ with the expression

$$
j_{*}=\left[\frac{2^{m_{1}(p-1)(2-p)}}{\delta_{1} v_{5}}\right]+1
$$

such that $\bar{t} \in\left(\tilde{t}, t^{\left(j_{*}\right)}\right]$. We now state the following proposition, which is the main result in this section.

Proposition 6.13 Suppose that assumptions (6.31) and (6.75) are in force. Then, there exists a constant s depending only upon the data and $\omega$ such that either

$$
u(x, t)<\mu_{+}-\frac{\omega}{2^{s}} \text { a.e. in } Q\left(d\left(\frac{R}{2}\right)^{p}, d_{*}\left(\frac{R}{2}\right)\right), \quad \text { or } \omega \leq\left(\frac{\bar{C}}{\ln \ln \ln \ln R^{-\kappa}}\right)^{\bar{\alpha}}
$$

for some constants $\kappa, \bar{C}$ and $\bar{\alpha}$, depending only upon the data and p. Moreover, $s \uparrow+\infty$ as $\omega \downarrow 0$.

Proof The main idea of the proof is to use the iteration in Sect. 6.5. To perform the iteration, observe that the set

$$
\bigcup_{k=0}^{j_{*}} K_{R^{(k)}} \times\left(t^{(k-1)}, t^{(k)}\right) \cap \Omega \times(-\infty, \bar{t}]
$$

must be contained in the reference cylinder $\bar{Q}=Q\left(R, R^{\frac{1}{p}}\right)$. To this end, we distinguish between the two cases $2^{j_{*}} \leq 20^{-1} R^{-\kappa}$ and $2^{j_{*}}>20^{-1} R^{-\kappa}$, where $\kappa=\frac{1}{2}\left(p-1-\frac{1}{p}\right)$.

In the first case, we observe that $K_{20 R^{\left(j_{*}\right)}} \subset K_{R^{\frac{1}{p}}}$. If there exists $j \in \mathbb{N}^{+}$such that $\bar{t}=t^{(j)}$, then $j<j_{*}$ and (6.74) holds. If $\bar{t} \neq t^{(j)}$ for any $j \geq 0$, then there exists $\bar{j} \in \mathbb{N}^{+}$ such that $t^{(\bar{j})}<\bar{t}<t^{(\bar{j}+1)}$ and $\bar{j}+1<j_{*}$. The iteration scheme requires only a slight modification. The process in Sect. 6.4 can be iterated $\bar{j}$ times to yield that (6.68) holds for $k=\bar{j}$. Starting from this estimate, we repeat the arguments from step 1 to step 2 in Sect. 6.5 to obtain (6.73) with $k=\bar{j}$. Furthermore, we can repeat the arguments in step 3 and step 4 in Sect. 6.5 with $t^{(k+1)}=\bar{t}$ since the proof remains effective with the condition $t^{(k+1)}=t^{(k)}+\delta_{1} v_{5} \omega^{(1-p)(2-p)} M_{1}^{k} R^{p}$ replaced by

$$
t^{(k)}<t^{(k+1)}<t^{(k)}+\delta_{1} v_{5} \omega^{(1-p)(2-p)} M_{1}^{k} R^{p} .
$$


It follows that

$$
u(x, t)<\mu_{+}-\frac{\omega}{2^{S} \tilde{j}+1} \quad \text { a.e. in } K_{\frac{1}{4} c_{2} R} \times(\tilde{t}, \bar{t}]
$$

and the proposition follows by setting $s=s_{j_{*}}$.

We now turn our attention to the case $2^{j_{*}}>20^{-1} R^{-\kappa}$. In view of (6.76), there exists a constant $C^{\prime}$ depending only upon the data and $p$ such that

$$
\exp \left\{\exp \left(C^{\prime} m_{2}\right)\right\} \geq R^{-\kappa},
$$

and the constant $C^{\prime}$ is stable as $p \rightarrow 2$. From this and taking into account (6.32), we conclude that

$$
\omega \leq\left(\frac{\bar{C}}{\ln \ln \ln \ln R^{-\kappa}}\right)^{\bar{\alpha}}
$$

for constants $\bar{C}$ and $\bar{\alpha}$ depending only upon the data and $p$. This completes the proof of Proposition 6.13.

\section{Proof of the main result concluded}

As we discussed in Sect. 4, the strategy of the proof is to study the oscillation of the weak solution in a sequence of nested and shrinking cylinders with a common vertex and prove that the essential oscillation converges to zero. We follow the notation used in Sect. 4 and assume that the common vertex coincides with $(0,0)$. To start with, we set $R_{1}=R$,

$$
Q_{1}=Q\left(\left(\frac{\omega}{2^{m_{1}}}\right)^{(1-p)(2-p)}\left(\frac{R_{1}}{2}\right)^{p},\left(\frac{\omega}{2^{m_{2}}}\right)^{p-2}\left(\frac{R_{1}}{2}\right)\right) \text { and } \bar{Q}_{1}=Q\left(R_{1}, R_{1}^{\frac{1}{p}}\right)
$$

From Propositions 5.4 and 6.13, we conclude that there exists a quantity $\sigma(\omega)$ such that either

$$
\underset{Q_{1}}{\operatorname{ess} \operatorname{osc}} u \leq \sigma(\omega) \omega, \quad Q_{1} \subset \bar{Q}_{1}, \quad \text { or } \quad \omega \leq\left(\frac{\bar{C}}{\ln \ln \ln \ln R_{1}^{-\kappa}}\right)^{\bar{\alpha}}
$$

where the constants $\bar{C}$ and $\bar{\alpha}$ depend only upon the data and $p$. Moreover, the quantity $\sigma(\omega)$ satisfies $\sigma(\omega)<1$ and $\sigma(\omega) \uparrow 1$ as $\omega \downarrow 0$. Set $R_{2}=2^{-p} R_{1}^{p}$ and $\omega_{1}=\sigma(\omega) \omega$. We proceed to construct the reference parabolic cylinder $\bar{Q}_{2}=Q\left(R_{2}, R_{2}^{\frac{1}{p}}\right)$. It is easy to check that $\bar{Q}_{2} \subset Q_{1} \cap \bar{Q}_{1}$ and ess osc $u \leq \omega$. Furthermore, we choose the intrinsic parabolic cylinder $Q_{2}$ by

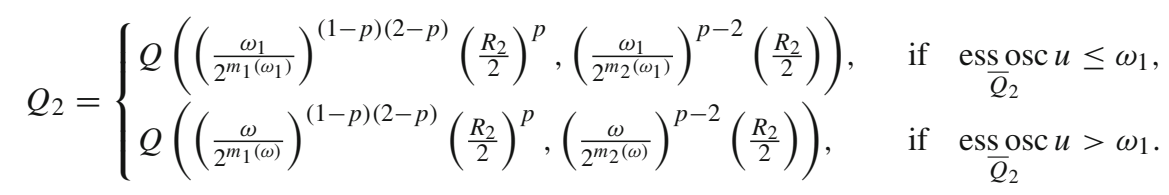


At this point, we apply Propositions 5.4 and 6.13 again, with $Q_{1}$ and $\bar{Q}_{1}$ replaced by $Q_{2}$ and $\bar{Q}_{2}$. We conclude that either

$$
\begin{aligned}
& \text { ess osc } u \leq \sigma\left(\omega_{1}\right) \omega_{1}, \quad Q_{2} \subset \bar{Q}_{2} \text {, } \\
& \text { or } \underset{\bar{Q}_{2}}{\operatorname{ess} \operatorname{osc}} u \leq \omega_{1}, \quad \omega_{1} \leq\left(\frac{\bar{C}}{\ln \ln \ln \ln R_{2}^{-\kappa}}\right)^{\bar{\alpha}} \text {, } \\
& \text { or } \quad \underset{Q_{2}}{\operatorname{ess} \operatorname{osc}} u \leq \sigma(\omega) \omega, \quad Q_{2} \subset \bar{Q}_{2} \text {, } \\
& \text { or } \omega \leq\left(\frac{\bar{C}}{\ln \ln \ln \ln R_{2}^{-\kappa}}\right)^{\bar{\alpha}} \text {, }
\end{aligned}
$$

for the same constants $\bar{C}$ and $\bar{\alpha}$ as in (7.1). We continue this process to find two sequences $\left\{R_{n}\right\}_{n=1}^{\infty}$ and $\left\{\omega_{n}\right\}_{n=1}^{\infty}$ such that

$$
R_{n+2}=2^{-p} R_{n+1}^{p} \quad \text { and } \quad \omega_{n+1}=\sigma\left(\omega_{n}\right) \omega_{n}, \quad n=1,2, \ldots
$$

Clearly, $R_{n} \downarrow 0$ as $n \rightarrow \infty$. Recalling the assumption $\omega>0$, it can be verified that $\omega_{n}>0$ for all $n \in \mathbb{Z}^{+}$. From [9, page 222], we see that $\omega_{n} \downarrow 0$ as $n \rightarrow \infty$. With these choices, we define $\bar{Q}_{n+1}=Q\left(R_{n+1}, R_{n+1}^{\frac{1}{p}}\right)$ and determine the intrinsic parabolic cylinder $Q_{n+1}$ by

$$
Q_{n+1}=\left\{\begin{array}{cc}
Q\left(\left(\frac{\omega_{n}}{2^{m_{1}\left(\omega_{n}\right)}}\right)^{(1-p)(2-p)}\left(\frac{R_{n+1}}{2}\right)^{p},\left(\frac{\omega_{n}}{2^{m_{2}\left(\omega_{n}\right)}}\right)^{p-2}\left(\frac{R_{n+1}}{2}\right)\right), & \text { if } \quad \overline{\operatorname{ess}}_{\bar{Q}_{n+1}} \operatorname{osc} u \leq \omega_{n}, \\
Q\left(\left(\frac{\omega_{n-1}}{2^{m_{1}\left(\omega_{n-1}\right)}}\right)^{(1-p)(2-p)}\left(\frac{R_{n+1}}{2}\right)^{p},\left(\frac{\omega_{n-1}}{2^{m_{2}\left(\omega_{n-1}\right)}}\right)^{p-2}\left(\frac{R_{n+1}}{2}\right)\right), & \text { if } \quad \omega_{n}<\frac{\operatorname{ess} \operatorname{osc} u \leq \omega_{n-1},}{Q_{n+1}} \\
\vdots\left(\left(\frac{\omega}{2^{m_{1}(\omega)}}\right)^{(1-p)(2-p)}\left(\frac{R_{n+1}}{2}\right)^{p},\left(\frac{\omega}{2^{m_{2}(\omega)}}\right)^{p-2}\left(\frac{R_{n+1}}{2}\right)\right), & \text { if } \quad \omega_{1}<\frac{\operatorname{ess} \operatorname{osc} u \leq \omega,}{Q_{n+1}}
\end{array}\right.
$$

where $n=2,3, \ldots$ These cylinders are nested with the common vertex $(0,0)$ and shrink to this point as $n \rightarrow \infty$. For a fixed $l \in \mathbb{Z}^{+}$, we remark that there is no subsequence $\left\{n_{k}\right\}_{k=1}^{\infty} \subset \mathbb{Z}^{+}$with $n_{k} \uparrow \infty$ as $k \rightarrow \infty$, such that

$$
\omega_{l} \leq\left(\frac{\bar{C}}{\ln \ln \ln \ln R_{n_{k}}^{-\kappa}}\right)^{\bar{\alpha}}, \text { for all } k=1,2, \ldots
$$

If this estimate holds true for any $k=1,2, \ldots$, then by passing to the limit $k \rightarrow \infty, \omega_{l}=0$ holds, which leads to a contradiction. Through repeated application of Propositions 5.4 and 6.13 , we conclude that there exist subsequences $\left\{n_{k}\right\}_{k=1}^{\infty}$ and $\left\{n_{k}^{\prime}\right\}_{k=1}^{\infty}$ such that either

$$
\underset{Q_{n_{k}}}{\operatorname{ess} \operatorname{osc}} u \leq \omega_{n_{k}^{\prime}}, \quad \text { or } \quad \bar{Q}_{n_{k}}^{\operatorname{ess}} \text { osc } u \leq \omega_{n_{k}^{\prime}} \text {. }
$$

There exists a sequence $\left\{r_{k}\right\}_{k=1}^{+\infty}$ with $r_{k} \downarrow 0$ as $k \rightarrow \infty$, such that ess osc $Q\left(r_{k}^{p}, r_{k}\right) u \downarrow 0$ as $k \rightarrow \infty$. Therefore, we conclude that the weak solutions are locally continuous, provided that $p$ satisfies (6.75). This completes the proof of Theorem 2.2.

Acknowledgements The author wishes to thank Eurica Henriques, Peter Lindqvist, Irina Markina and José Miguel Urbano for the valuable discussions. The author would also like to thank the referees for providing helpful comments to improve the manuscript. 


\section{References}

1. Alkhutov, YuA, Zhikov, V.V.: Hölder continuity of solutions of parabolic equations with variable nonlinearity exponent. (Translated from Trudy Seminara imeni I. G. Petrovskogo, No. 28, Part I, pp. 8-74, 2011)(Translated from Trudy Seminara imeni I. G. Petrovskogo, No. 28, Part I, pp. 8-74, 2011). J. Math. Sci. (N. Y.) 179, 347-389 (2011)

2. Caffarelli, L.A., Evans, L.C.: Continuity of the temperature in the two-phase Stefan problem. Arch. Ration. Mech. Anal. 81, 199-220 (1983)

3. Chen, Y.Z., DiBenedetto, E.: On the local behaviour of solutions of singular parabolic equations. Arch. Ration. Mech. Anal. 103, 319-345 (1988)

4. DiBenedetto, E.: Continuity of weak solutions to certain singular parabolic equations. Ann. Mat. Pura Appl. 130, 131-176 (1982)

5. DiBenedetto, E.: Degenerate Parabolic Equations. Springer, New York (1993)

6. Henriques, E., Urbano, J.M.: On the Doubly singular equation $\gamma(u)_{t}=\Delta_{p} u$. Commun. Partial Differ. Equ. 30(5-6), 919-955 (2005)

7. Ladyzhenskaya, O.A., Solonnikov, V.A., Uraltseva, N.N.: Linear and Quasilinear Equations of Parabolic Type, vol. 23. American Mathematical Society, Providence (1968)

8. Rodrigues, J.F.: Variational methods in the Stefan problem. In: Visintin, A. (ed.) Phase Transitions and Hysteresis. Lecture Notes in Mathematics, vol. 1584, pp. 147-212. Springer, Berlin (1994)

9. Urbano, J.M.: A free boundary problem with convection for the $p$-Laplacian. Rend. Mat. Appl. 17, 1-19 (1997)

10. Urbano, J.M.: Continuous solutions for a degenerate free boundary problem. Ann. Mat. Pura Appl. 178, 195-224 (2000)

Publisher's Note Springer Nature remains neutral with regard to jurisdictional claims in published maps and institutional affiliations. 